Health Services Research

\title{
$\Theta$ Trends of Expenditures and Utilization of Facet Joint Interventions in Fee-For-Service (FFS) Medicare Population from 2009-2018
}

\author{
Laxmaiah Manchikanti, MD1, Vidyasagar Pampati, MSc², Amol Soin, MD³, \\ Rachana Vanaparthy, MBBS ${ }^{4}$, Mahendra R. Sanapati, MD, Alan D. Kaye, MD, $\mathrm{PhD}^{6}$, \\ and Joshua A. Hirsch, MD
}

From: ${ }^{1,2}$ Pain Management Centers of America, Paducah, KY; ${ }^{3}$ Ohio Pain Clinic, Centerville, $\mathrm{OH}$, Wright State University, Dayton, $\mathrm{OH} ;{ }^{4}$ Oregon Health and Science University, Portland, OR; ' 5 Pain Management Centers of America, Evansville, IN; ${ }^{6}$ LSU School of Medicine, Shreveport, LA and New Orleans, LA and Tulane School of Medicine, New Orleans, LA; ${ }^{7}$ Massachusetts General Hospital and Harvard Medical School, Boston, MA

See Author Affiliations on P. S143.

Address Correspondence: Laxmaiah Manchikanti, MD 67 Lakeview Drive Paducah, Kentucky 42001

E-mail: drlm@thepainmd.com

Disclaimer: There was no external funding in the preparation of this manuscript.Conflict of interest: Dr. Kaye is a speaker for Merck.

Dr. Hirsch is a consultant for

Medtronic and Senior Affiliate Research Fellow at the Neiman Policy Institute.

Manuscript received: 04-28-2020 Revised manuscript received: 05-15-2020

Accepted for publication: 05-22-2020

Free full manuscript: www.painphysicianjournal.com
Background: The trends of the expenditures of facet joint interventions have not been specifically assessed in the fee-for-service (FFS) Medicare population since 2009

Objectives: The objective of this investigation is to assess trends of expenditures and utilization of facet joint interventions in FFS Medicare population from 2009 to 2018.

Study Design: The study was designed to analyze trends of expenditures and utilization of facet joint interventions in FFS Medicare population from 2009-2018 in the United States. In this manuscript:

- A patient was considered as undergoing facet joint interventions throughout the year.

- A visit included all regions treated during the visit.

- An episode was considered as one per region utilizing primary codes only.

- Services or procedures were considered all procedures (multiple levels).

Data for the analysis was obtained from the standard $5 \%$ national sample of the Centers for Medicare \& Medicaid Services (CMS) physician outpatient billing claims for those enrolled in the FFS Medicare program from 2009 to 2018. All the expenditures were presented with allowed costs and also were inflation adjusted to 2018 US dollars.

Results: This analysis showed expenditures increased by $79 \%$ from 2009 to 2018 in the form of total cost for facet joint interventions, at an annual rate of $6.7 \%$. Cervical and lumbar radiofrequency neurotomy procedures increased $185 \%$ and $169 \%$. However, inflation-adjusted expenditures with 2018 US dollars showed an overall increase of $53 \%$ with an annual increase of $4.9 \%$. In addition, using inflation-adjusted expenditures per procedures increased, the overall $6 \%$ with an annual increase of $0.7 \%$. Overall, per patient costs, with inflation adjustment, decreased from $\$ 1,925$ to $\$ 1,785$ with a decline of $7 \%$ and an annual decline of $0.8 \%$. Allowed charges per visit also declined after inflation adjustment from $\$ 951.76$ to $\$ 849.86$ with an overall decline of $11 \%$ and an annual decline of $1.3 \%$. Staged episodes of radiofrequency neurotomy were performed in $23.9 \%$ of patients and more than 2 episodes for radiofrequency neurotomy in $6.9 \%$, in lumbar spine and $19.6 \%$ staged and $5.1 \%$ more than 2 episodes in cervical spine of patients in 2018

Limitations: This analysis is limited by inclusion of only the FFS Medicare population, without adding utilization patterns of Medicare Advantage plans, which constitutes almost $30 \%$ of the Medicare population.

Conclusions: Even after adjusting for inflation, there was a significant increase for the expenditures of facet joint interventions with an overall $53 \%$ increase. Costs per patient and cost per visit declined. Inflation-adjusted cost per year declined $7 \%$ overall and $0.8 \%$ annually from $\$ 1,925$ to $\$ 1,785$, and inflation-adjusted cost per visit also declined $11 \%$ annually and $1.3 \%$ per year from $\$ 952$ in 2009 to $\$ 850$ in 2018 .

Key words: Facet joint interventions, facet joint nerve blocks, facet joint neurolysis, facet joint injections, Medicare expenditures

Pain Physician 2020: 23:S129-S147 


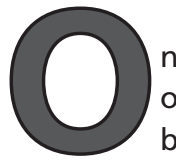

nce escalating and now flattening, utilization of facet joint interventions continues to be debated and is associated with multiple policy changes and regulations. The recent analyses of utilization patterns of interventional techniques in general $(1,2)$ and facet joint interventions in particular (3-5) have shown significant alterations in utilization patterns. While there was a decline in utilization of various interventional techniques, facet joint interventions stayed flat with a decline for some procedures, but with an increase in others. Overall, the proportion of facet joint interventions to all interventional techniques was $25.5 \%$ in 2000 , increasing to $39.5 \%$ in 2009 and $46.8 \%$ in $2018(1,3)$. During the same period, epidurals declined from $59 \%$ in 2000 to $46 \%$ in 2009 and $39 \%$ in 2018. Facet joint interventions include facet joint nerve blocks or intraarticular injections and radiofrequency neurotomy procedures. Facet joint interventions in the Medicare population, has increased by $18.8 \%$ from 2009 to 2018 per 100,000 fee-for-service (FFS) with an annual increase of $1.9 \%$, and a significant decline compared to an overall increase of $309.9 \%$ from 2000 to 2009 during which there was an annual increase of $17 \%$ (3). The importance of this is that lumbosacral facet joint nerve block visits or sessions decreased at an annual rate of $0.2 \%$ from 2009 to 2018, with an increase of $15.2 \%$ from 2000 to 2009 . In contrast, lumbosacral facet joint neurolysis sessions increased at an annual rate of $7.4 \%$ from 2009 to 2018 , compared to an annual increase rate of $23 \%$ from 2000 to 2009 , with reduction in growth patterns. Further, compared to lumbosacral facet joint nerve block episodes, which showed a decline at an annual rate of $0.2 \%$ from 2009 to 2018 , this is a disproportionate increase. The ratio of lumbar facet joint nerve block sessions to lumbosacral facet joint neurolysis episodes decreased from 6.7 in 2000 to 1.9 in 2018. Cervical and thoracic facet joint injection episodes increased at an annual rate of $0.5 \%$ compared with cervicothoracic facet neurolysis episodes of $8.7 \%$ from 2009 to 2018. Cervical and thoracic facet joint injection episodes increased at an annual rate of $0.5 \%$ compared with cervicothoracic facet neurolysis episodes of $8.7 \%$ from 2009 to 2018 . Cervical facet joint injections increased $4.9 \%$ from 2009 to 2018 compared with neurolysis procedures increase of $112 \%$. The ratio of cervical facet joint injection sessions to neurolysis episodes changed from 8.9 in 2000 to 2.4 in 2018. This pattern of utilization significantly changed since the enactment of new local coverage determinations (LCDs) which tended to shift procedures towards neurolysis and away from facet joint nerve blocks (6-8).

Similar observations were also made by Starr et al (5) in patients with commercial insurance with utilization and cost of lumbar radiofrequency ablation and lumbar facet joint injections. The results of this analysis showed that from 2007 to 2016, lumbar radiofrequency sessions performed per 100,000 enrollees per year increased from 49 to 113 , a $130.6 \%$ overall increase and $9.7 \%$ annual increase. During the same period, lumbar facet joint injection use increased at a slower pace from 201 to 251 sessions per 100,000 enrollees, a $24.9 \%$ overall increase versus $130.6 \%$ overall increase and an annual increase of $2.5 \%$ compared to $9.7 \%$ for radiofrequency ablation. Surprisingly, they also showed that in the year after lumbar facet joint injections, less than $27 \%$ of patients received lumbar radiofrequency ablation, almost $29 \%$ received another injection, but not radiofrequency ablation, almost $45 \%$ of the patients received neither. The cost for lumbar facet joint nerve blocks per 100,000 enrollees went from $\$ 257,280$ in 2007 to $\$ 396,580$ in 2016 , a $4.9 \%$ annual increase, whereas for facet joint radiofrequency ablation, costs increased from $\$ 94,570$ in 2007 to $\$ 206,680$ in 2016, an annual increase of $12.2 \%$. Thus, reducing lumbar facet joint injections does not seem to reduce the overall utilization patterns or even the costs.

Manchikanti et al (2) assessed the growth of spinal interventional pain management techniques, along with Medicare expenditures from 2000 to 2008. In previous analysis, the authors did not look at facet joint interventions and their costs individually. However, they calculated the total costs of interventional procedures, including epidural injections, adhesiolysis procedures, facet joint interventions, and sacroiliac joint interventions. The overall costs for interventional techniques increased from $\$ 362,347,025$ in 2000 to $\$ 1,231,180,420$ in 2008 , a $240 \%$ increase, with an increase of $43 \%$ per patient, $28 \%$ per visit, and $3 \%$ per procedure code.

Heath care costs are a concern for all of the US population. In fact, in 2016, low back and neck pain expenditures increased an estimated $\$ 134.5$ billion, and $\$ 129.8$ billion for musculoskeletal conditions, with total spending of $\$ 264.3$ billion, an increase of $44.4 \%$, from $\$ 183$ billion in $2013(9,10)$. At the same time, overall US healthcare spending has reached $\$ 3.65$ trillion in 2018, with per person costs for health care increasing to $\$ 11,012$ in $2018(11,12)$. More importantly, in 2018, 
Medicare benefit payments totaled $\$ 731$ billion, up from $\$ 462$ billion in 2008. Part B spending consisting of physician services and hospital outpatient services increased from $39 \%$ to $46 \%$, whereas Part A benefits consisting of mainly hospital inpatient services decreased from $50 \%$ to $41 \%$, with Part D prescription drug benefits increasing from $11 \%$ to $13 \%$ (13). Further, costs of Medicare are expected to increase rapidly in upcoming years (14). Unfortunately, all these estimations look very optimistic considering the economic and health impact of COVID-19 (15-17). Thus, utilization patterns and costs continue to be a major issue for facet joint interventions. Other issues also include claims of lack of medical necessity, indications and lack of cost utility. Further, over the past 2 decades, multiple modalities in pain management have shown a significant escalation in utilization, including opioids, leading to an opioid epidemic and escalating deaths (18-39). However, appropriate systematic reviews and randomized controlled trials (RCTs) have shown significant evidence of efficacy for interventional techniques in general and facet joint interventions in particular, along with cost utility (4054). Consequently, multiple attempts have been made to control the utilization patterns of facet joint interventions, along with other interventional techniques by affecting coverage policies based on LCDs in Medicare populations, increased oversight from Medicare, coding changes, and reimbursement reductions (5-8,5561). Cost utility analysis of facet joint nerve blocks has shown similar cost effectiveness as epidurals and other interventional techniques $(42,43,50-52)$. These cost utilities were derived from surgical interventions from Spine Patient Outcomes Research Trial (SPORT) studies $(62,63)$. Even though opponents and proponents continue to come to discordant conclusions with negative and positive recommendations, the literature of the effectiveness and appropriateness of facet joint interventions continues to accumulate $(40,41,47,64-69)$.

This manuscript was undertaken to assess utilization patterns and expenditures of facet joint interventions from 2009 to 2018.

\section{Methods}

This retrospective cohort analysis of Medicare expenditures and utilization trends was performed as per the methodology as described by the Strengthening the Reporting of Observational Studies in Epidemiology (STROBE) (70).

The data for this study were obtained from the standard $5 \%$ national sample of the Centers for Medi- care \& Medicaid Services (CMS) physician outpatient billing claims for those enrolled in the FFS Medicare program for 2009 through 2018 (71). The CMS 5\% sample data set is considered to be unbiased and unpredictable in terms of any patient characteristics, but does allow appropriate tracking of patients over time and across databases. Thus, institutional review board (IRB) approval was not required.

\section{Study Design}

This analysis of utilization patterns of facet interventions was designed as a retrospective cohort study in FFS Medicare population in the United States calculating trends of utilization and costs from 2009 to 2018 (71) in the United States. In this analysis:

- A patient was considered as undergoing facet joint interventions throughout the year, irrespective of visits, irrespective of number of visits, episodes or services.

- A visit included all regions treated during the visit.

- An episode was considered as one per region utilizing primary codes only.

- Services or procedures were considered all procedures (multiple levels) including add-on codes.

- Staged episodes were assessed based on if radiofrequency neurotomy was performed within 3 months. A staged episode is defined as an episode repeated in the same region; however, on a different side or involving different joints before 6 months elapsed.

- Number of episodes beyond allowed number based on LCDs was considered as more than approved episodes.

\section{Setting}

The setting of this analysis involved review of the standard $5 \%$ national sample of CMS services physician outpatient billing claims for those enrolled in FFS Medicare program from 2009 to 2018. Participants included Medicare FFS recipients receiving facet joint interventions. The Current Procedural Terminology (CPT) codes included in this analysis are listed in Table 1.

\section{Data Sources}

Data were obtained from CMS physician outpatient billing claims for those enrolled in the FFS Medicare program from 2009 to 2018.

\section{Data Compilation}

Data were compiled utilizing Microsoft 365 Access and Microsoft 365 Excel (Microsoft, Redmond, WA). 
Table 1. CPT codes utilized for facet joint interventions from 2009 to 2018.

\begin{tabular}{|c|c|}
\hline CPT CODE & DESCRIPTION \\
\hline \multicolumn{2}{|c|}{ CPT CODES UNTIL 2009} \\
\hline 64470 & Injection, anesthetic agent and/or steroid, paravertebral facet joint or facet joint nerve; cervical or thoracic, single level \\
\hline 64472 & Injection, anesthetic agent and/or steroid, paravertebral facet joint or facet joint nerve; cervical or thoracic, each additional level \\
\hline 64475 & Injection, anesthetic agent and/or steroid, paravertebral facet joint or facet joint nerve; lumbar or sacral, single level \\
\hline 64476 & Injection, anesthetic agent and/or steroid, paravertebral facet joint or facet joint nerve; lumbar or sacral, each additional level \\
\hline \multicolumn{2}{|c|}{ CPT CODES FROM 2010 TO 2018} \\
\hline 64490 & $\begin{array}{l}\text { Injection(s), diagnostic or therapeutic agent, paravertebral facet (zygapophyseal) joint (or nerves innervating that joint) with } \\
\text { image guidance (fluoroscopy or CT), cervical or thoracic; single level }\end{array}$ \\
\hline 64491 & $\begin{array}{l}\text { Injection(s), diagnostic or therapeutic agent, paravertebral facet (zygapophyseal) joint (or nerves innervating that joint) } \\
\text { with image guidance (fluoroscopy or CT), cervical or thoracic; second level (List separately in addition to code for primary } \\
\text { procedure) }\end{array}$ \\
\hline 64492 & $\begin{array}{l}\text { Injection(s), diagnostic or therapeutic agent, paravertebral facet (zygapophyseal) joint (or nerves innervating that joint) with } \\
\text { image guidance (fluoroscopy or CT), cervical or thoracic; third and any additional level(s) (List separately in addition to code } \\
\text { for primary procedure) }\end{array}$ \\
\hline 64493 & $\begin{array}{l}\text { Injection(s), diagnostic or therapeutic agent, paravertebral facet (zygapophyseal) joint (or nerves innervating that joint) with } \\
\text { image guidance (fluoroscopy or CT), lumbar or sacral; single level }\end{array}$ \\
\hline 64494 & $\begin{array}{l}\text { Injection(s), diagnostic or therapeutic agent, paravertebral facet (zygapophyseal) joint (or nerves innervating that joint) with } \\
\text { image guidance (fluoroscopy or CT), lumbar or sacral; second level (List separately in addition to code for primary procedure) }\end{array}$ \\
\hline 64495 & $\begin{array}{l}\text { Injection(s), diagnostic or therapeutic agent, paravertebral facet (zygapophyseal) joint (or nerves innervating that joint) with } \\
\text { image guidance (fluoroscopy or CT), lumbar or sacral; third and any additional level(s) (List separately in addition to code for } \\
\text { primary procedure) }\end{array}$ \\
\hline \multicolumn{2}{|c|}{ CPT CODES FROM 2012 TO 2018} \\
\hline 64633 & $\begin{array}{l}\text { Destruction by neurolytic agent, paravertebral facet joint nerve(s), with imaging guidance (fluoroscopy or CT); cervical or } \\
\text { thoracic, single facet joint }\end{array}$ \\
\hline 64634 & $\begin{array}{l}\text { Destruction by neurolytic agent, paravertebral facet joint nerve(s), with imaging guidance (fluoroscopy or CT); cervical or } \\
\text { thoracic, each additional facet joint (List separately in addition to code for primary procedure) }\end{array}$ \\
\hline 64635 & $\begin{array}{l}\text { Destruction by neurolytic agent, paravertebral facet joint nerve(s), with imaging guidance (fluoroscopy or CT); lumbar or } \\
\text { sacral, single facet joint }\end{array}$ \\
\hline 64636 & $\begin{array}{l}\text { Destruction by neurolytic agent, paravertebral facet joint nerve(s), with imaging guidance (fluoroscopy or CT); lumbar or } \\
\text { sacral, each additional facet joint }\end{array}$ \\
\hline \multicolumn{2}{|c|}{ CPT CODES FROM 2009TO 2012} \\
\hline 64622 & Destruction by neurolytic agent, paravertebral facet joint nerve; lumbar or sacral, single level \\
\hline 64623 & Destruction by neurolytic agent, paravertebral facet joint nerve; lumbar or sacral, each additional level \\
\hline 64626 & Destruction by neurolytic agent, paravertebral facet joint nerve; cervical or thoracic, single level \\
\hline 64627 & Destruction by neurolytic agent, paravertebral facet joint nerve; cervical or thoracic, each additional level \\
\hline
\end{tabular}

We removed all facet joint inventions services with zero allowed payments. One hundred percent data was obtained by multiplication with 20 to scale up from our $5 \%$ sample to the full M-FFS population. The data were calculated for overall services for each procedure, and the rate of services, based on utilization per 100,000 FFS Medicare beneficiaries. Expenditures were also calculated for physician and facility, which included allowable charges for physician, and facility (ASC, HOPD, office setting). All the expenditures were presented with allowed costs and also were adjusted with inflation to 2018 US dollars. HOPD facility allowed charges were estimated based on National Average rates.

\section{Variables}

The analysis of trends of utilization and costs patterns of facet joint interventions incorporated multiple variables with analysis and costs for all procedures, utilization based on statewide and Medicare Administrative Contractors (MACs) and location of the service 
provided, either office-based, ambulatory surgery center (ASC)-based, or hospital outpatient department (HOPD)-based.

\section{Measures}

Allowed services were assessed for each procedure, and rates were calculated based on Medicare beneficiaries for the corresponding year and are reported as procedures per 100,000 Medicare beneficiaries. Data were assessed for total number of procedures performed, as well as number of visits or sessions for lumbar facet joint interventions. A session or episode is considered as one per region, irrespective of number of procedures performed. More than 2 radiofrequency neurotomy episodes and staged episodes were assessed for 2018.

\section{Bias}

Data were purchased from the CMS by American Society of Interventional Pain Physicians (ASIPP). The study was conducted with the internal resources of the primary author's practice without external funding. The costs were determined without eliciting any bias. Thus, based on the large size of the dataset derived from a government source, there was no information related to patient individual identification.

\section{Sample Size}

The size of this retrospective cohort study is considered to be large, providing real-world claims data on Medicare patients with inclusion of all Medicare FFS patients undergoing facet joint interventions for spinal pain from 2009 to 2018.

\section{Results}

\section{Participants and Characteristics}

Participants in this assessment of trends in expenditures and utilization of facet joint interventions from 2009 to 2018 included all Medicare FFS recipients. Table 2 shows descriptive data of facet joint interventions and population characteristics.

The number of patients receiving facet joint interventions showed a dramatic increase from 463,500 to 797,460 from 2009 to 2018, an overall increase of $65.1 \%$ or an annual increase of $5.7 \%$. The number of patients per 100,000 population annual increase of rate of visits, rate of episodes of treatment, and rate of procedures increased $2.7 \%, 3.1 \%, 2.2 \%$, and $1.1 \%$ respectively.

Table 3 shows utilization patterns and ratios of lumbar facet joint nerve blocks compared to facet joint neurolysis and cervical/thoracic facet joint nerve blocks compared to facet neurolysis in Medicare population from 2009 to 2018. These ratios changed from 3.8 in 2009 to 1.9 in 2018 for lumbar facet joint interventions, whereas, for cervical/thoracic facet joint interventions, the ratio changed from 4.9 to 2.4 .

Figure 1 shows facet joint interventions data by services, visits and patients per 100,000 FFS Medicare population.

Figure 2 shows utilization patterns for nerve blocks and radiofrequency neurolysis procedures.

\section{Expenditure Characteristics}

Table 4 shows total allowed charges by place of services by type of procedures showing significant growth of facet joint radiofrequency in all settings; however, the increases for cervical facet joint and lumbar facet joint injections were $35 \%$ and $37 \%$ compared to increases of cervical radiofrequency neurotomy of $185 \%$ and lumbar radiofrequency neurotomy of $169 \%$, with a total increase of costs of 79\% from 2009 to 2018. The inflation-adjusted costs to 2018 showed an overall increase of $53 \%$ compared to $79 \%$ without adjustment and at an annual increase of $4.9 \%$ compared to $6.7 \%$ without an adjustment. This table also shows costs for 100,000 Medicare beneficiaries with costs per beneficiary which increased over a period of time by $18 \%$ without adjusting for inflation. It also shows per beneficiary costs of $\$ 13$ in 2009 , increasing to $\$ 15$, an $18 \%$ increase per beneficiary, with an $1.8 \%$ annual increase.

Table 5 shows average allowed charges per patient; the highest amount was in HOPD settings at $\$ 2,746.64$ in 2009 , changing slightly to $\$ 2,798.50$ in 2018 , a $2 \%$ increase or an $0.2 \%$ annual increase. In ASC settings, the fees were much lower. Overall allowed charges were lower than ASCs with a total of $\$ 1,775.54$ in 2009, increasing to $\$ 1,855.61$, with an annual increase of $0.5 \%$ and a total increase of $5 \%$. In contrast, for office procedures, the increases were slightly higher at $8 \%$ total and $0.8 \%$ annually from $\$ 1,026.86$ in 2009 to $\$ 1,107.36$ in 2018, significantly lower than hospitals outpatient departments and ASCs. Overall, on average, total payments per patient were $\$ 1,645.29$ in 2009 increasing to $\$ 1,785.31$, a $9 \%$ increase overall with $0.9 \%$ increase per year. Inflation-adjusted costs demonstrated increases from $\$ 1,645.29$ to $\$ 1,924.99$ in 2009 , resulting in an overall decline of $0.7 \%$ of the allowed charges with an annual decline of $7 \%$ compared to overall increase of $9 \%$ and annual increase of $0.9 \%$ prior to adjustment of inflation. 
Pain Physician: May/June 2020 23:S129-S147

Table 2. Characteristics of Medicare beneficiaries and utilization pattern of facet joint interventions.

\begin{tabular}{|c|c|c|c|c|c|c|c|c|c|c|c|c|}
\hline Year & Y2009 & Y2010 & Y2011 & Y2012 & Y2013 & Y2014 & Y2015 & Y2016 & Y2017 & Y2018 & change & GM \\
\hline $\begin{array}{l}\text { U.S. } \\
\text { Population }\end{array}$ & 307,006 & 308,746 & 311,583 & 313,874 & 316,129 & 318,892 & 320,897 & 323,127 & 326,625 & 327,167 & $6.6 \%$ & $0.7 \%$ \\
\hline $\begin{array}{l}\text { Medicare } \\
\text { Beneficiaries }\end{array}$ & 45,801 & 46,914 & 48,300 & 50,300 & 51,900 & 53,500 & 54,900 & 56,500 & 58,000 & 59,600 & $30.1 \%$ & $3.0 \%$ \\
\hline$\geq 65$ years & 38,177 & 38,991 & 40,000 & 41,900 & 43,100 & 44,600 & 46,000 & 47,500 & 49,200 & 50,800 & $33.1 \%$ & $3.2 \%$ \\
\hline $\begin{array}{l}(\%>=65 \\
\text { years })\end{array}$ & $83.4 \%$ & $83.1 \%$ & $82.8 \%$ & $83.3 \%$ & $83.0 \%$ & $83.4 \%$ & $83.8 \%$ & $84.1 \%$ & $84.8 \%$ & $85.2 \%$ & & \\
\hline$<65$ years & 7,624 & 7,923 & 8,300 & 8,500 & 8,800 & 8,900 & 9,000 & 9,000 & 8,900 & 8,800 & $15.4 \%$ & $1.6 \%$ \\
\hline \multicolumn{13}{|c|}{ Facet joint Interventions } \\
\hline $\begin{array}{l}\text { Allowed } \\
\text { Services } \\
\text { (Procedures) }\end{array}$ & $1,860,600$ & $1,716,860$ & $1,800,300$ & $1,911,020$ & $1,946,180$ & $2,074,980$ & $2,283,980$ & $2,441,560$ & $2,565,900$ & $2,677,540$ & $43.9 \%$ & $4.1 \%$ \\
\hline Visits & 625,860 & 635,440 & 661,440 & 723,420 & 758,640 & 821,020 & 906,720 & 973,700 & $1,027,720$ & $1,073,500$ & $71.5 \%$ & $6.2 \%$ \\
\hline Rate & 1,366 & 1,354 & 1,369 & 1,438 & 1,462 & 1,535 & 1,652 & 1,723 & 1,772 & 1,801 & $31.8 \%$ & $3.1 \%$ \\
\hline \multicolumn{13}{|l|}{ Patients } \\
\hline$>=65$ years & 223,700 & 223,220 & 231,160 & 245,640 & 253,600 & 276,960 & 308,020 & 336,000 & 360,780 & 387,040 & $73.0 \%$ & $6.3 \%$ \\
\hline $\begin{array}{l}(\%>=65 \\
\text { years })\end{array}$ & $72.3 \%$ & $71.3 \%$ & $69.9 \%$ & $69.0 \%$ & $69.1 \%$ & $69.9 \%$ & $71.1 \%$ & $72.4 \%$ & $73.7 \%$ & $75.7 \%$ & & \\
\hline Rate & 488 & 476 & 479 & 488 & 489 & 518 & 561 & 595 & 622 & 649 & $33.0 \%$ & $3.2 \%$ \\
\hline$<65$ years & 85,740 & 89,720 & 99,500 & 110,580 & 113,260 & 119,080 & 125,500 & 127,900 & 128,540 & 123,980 & $44.6 \%$ & $4.2 \%$ \\
\hline Rate & 187 & 191 & 206 & 220 & 218 & 223 & 229 & 226 & 222 & 208 & $11.1 \%$ & $1.2 \%$ \\
\hline Total Patients & 309,440 & 312,940 & 330,660 & 356,220 & 366,860 & 396,040 & 433,520 & 463,900 & 489,320 & 511,020 & $65.1 \%$ & $5.7 \%$ \\
\hline Rate & 676 & 667 & 685 & 708 & 707 & 740 & 790 & 821 & 844 & 857 & $26.8 \%$ & $2.7 \%$ \\
\hline \multicolumn{13}{|c|}{ Episodes (primary codes only) } \\
\hline Rate & 1,476 & 1,389 & 1,407 & 1,476 & 1,469 & 1,536 & 1,649 & 1,714 & 1,764 & 1,795 & $21.6 \%$ & $2.2 \%$ \\
\hline \multicolumn{13}{|c|}{ Episodes based Age groups } \\
\hline$>=65$ & 463,500 & 443,700 & 453,280 & 488,860 & 502,200 & 548,920 & 620,400 & 679,400 & 735,340 & 797,460 & $72.1 \%$ & $6.2 \%$ \\
\hline Rate & 1,214 & 1,138 & 1,133 & 1,167 & 1,165 & 1,231 & 1,349 & 1,430 & 1,495 & 1,570 & $29.3 \%$ & $2.9 \%$ \\
\hline$<65$ & 212,360 & 208,020 & 226,100 & 253,680 & 260,220 & 272,800 & 285,000 & 289,260 & 287,560 & 272,340 & $28.2 \%$ & $2.8 \%$ \\
\hline Rate & 2,785 & 2,626 & 2,724 & 2,984 & 2,957 & 3,065 & 3,167 & 3,214 & 3,231 & 3,095 & $11.1 \%$ & $1.2 \%$ \\
\hline \multicolumn{13}{|c|}{ Episodes based on Place of Service } \\
\hline ASC & 160,560 & 166,400 & 180,020 & 208,340 & 205,000 & 225,340 & 257,180 & 283,980 & 305,060 & 326,120 & $103.1 \%$ & $8.2 \%$ \\
\hline Rate & 351 & 355 & 373 & 414 & 395 & 421 & 468 & 503 & 526 & 547 & $56.1 \%$ & $5.1 \%$ \\
\hline HOPD & 144,320 & 153,660 & 164,460 & 179,220 & 188,220 & 197,340 & 220,500 & 238,860 & 248,920 & 261,980 & $81.5 \%$ & $6.8 \%$ \\
\hline Rate & 315 & 328 & 340 & 356 & 363 & 369 & 402 & 423 & 429 & 440 & $39.5 \%$ & $3.8 \%$ \\
\hline Office & 370,980 & 331,660 & 334,900 & 354,980 & 369,200 & 399,040 & 427,720 & 445,820 & 468,920 & 481,700 & $29.8 \%$ & $2.9 \%$ \\
\hline Rate & 810 & 707 & 693 & 706 & 711 & 746 & 779 & 789 & 808 & 808 & $-0.2 \%$ & $0.0 \%$ \\
\hline
\end{tabular}

Table 6 shows average allowed charges per visit in all 3 settings. The data showed a $4 \%$ overall decline, or $0.4 \%$ annually for ASCs, $-7 \%$ or $-0.8 \%$ for HOPD, whereas for offices, it showed an increase of $7 \%$ with $0.8 \%$ annual rate. The reimbursements in 2019 ranged from
$\$ 468.71$ in office settings, $\$ 912.50$ in ASC settings, and $\$ 1,557.84$ in HOPD settings for total professional and facility fee in 2009 to $\$ 532$ in office settings, $\$ 877.86$ in ASC settings, and \$1,452.30 in HOPD settings in 2018. Average total cost prior to inflation adjustment per 
Trends of Expenditures of Facet Joint Interventions in Medicare Population

Table 3. Frequency of rate utilizations of facet joint interventions (facet nerve blocks vs facet neurolysis) in the Medicare population from 2009-2018.

\begin{tabular}{|c|c|c|c|c|c|c|}
\hline Year & $\begin{array}{c}\text { C/T Facet } \\
\text { Joint Block } \\
\text { (Only Primary } \\
\text { 64470/ 64490) }\end{array}$ & $\begin{array}{c}\text { C/T Facet } \\
\text { Neurolysis } \\
\text { (Only Primary } \\
\text { 64626/ 64633) }\end{array}$ & $\begin{array}{c}\text { Ratio of } \\
\text { C/T Facet Joint } \\
\text { NBs } \\
\text { over RFT }\end{array}$ & $\begin{array}{c}\text { L/S Facet } \\
\text { Joint Blocks } \\
\text { (Only Primary } \\
\text { 64475/ 64493) }\end{array}$ & $\begin{array}{c}\text { L/S Facet } \\
\text { Neurolysis } \\
\text { (Only Primary } \\
\text { 64622/ 64635) }\end{array}$ & $\begin{array}{c}\text { Ratio of L/S } \\
\text { Facet Joint } \\
\text { NBs over RFT }\end{array}$ \\
\hline F2009 & 278 & 57 & 4.9 & 901 & 240 & 3.8 \\
\hline F2010 & 257 & 56 & 4.6 & 826 & 250 & 3.3 \\
\hline F2011 & 262 & 62 & 4.2 & 820 & 262 & 3.1 \\
\hline F2012 & 262 & 69 & 3.8 & 859 & 286 & 3.0 \\
\hline F2013 & 265 & 74 & 3.6 & 822 & 308 & 2.7 \\
\hline F2014 & 275 & 80 & 3.4 & 849 & 332 & 2.6 \\
\hline F2015 & 285 & 93 & 3.1 & 896 & 375 & 2.4 \\
\hline F2016 & 293 & 103 & 2.8 & 907 & 411 & 2.2 \\
\hline F2017 & 290 & 110 & 2.6 & 911 & 452 & 2.0 \\
\hline F2018 & 293 & 121 & 2.4 & 906 & 475 & 1.9 \\
\hline Change & $5.6 \%$ & $111.9 \%$ & & $0.6 \%$ & $97.7 \%$ & \\
\hline GM & $0.6 \%$ & $8.7 \%$ & & $0.1 \%$ & $7.9 \%$ & \\
\hline
\end{tabular}

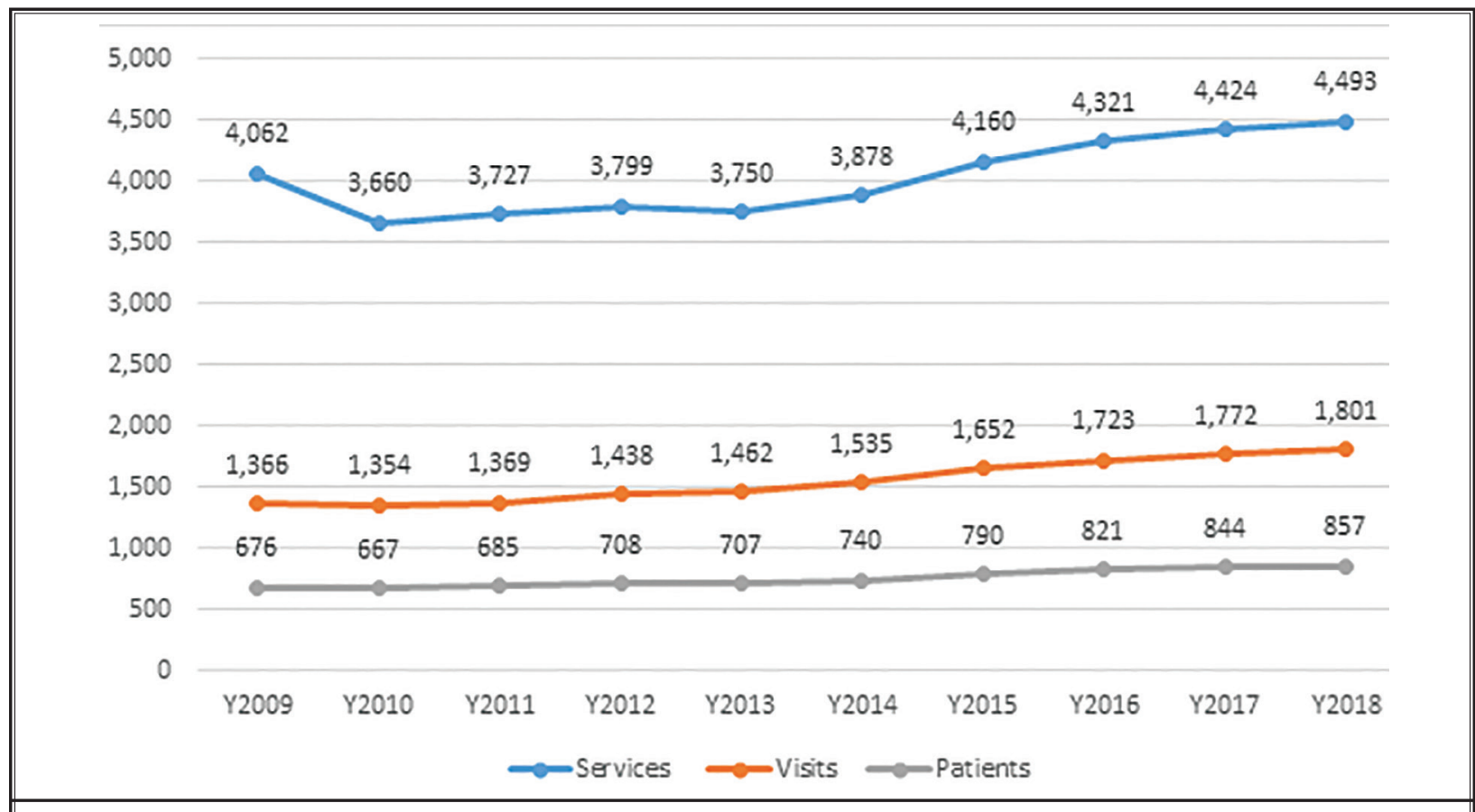

Fig. 1. Facet joint intervention rate per 100,000 FFS Medicare population by services, episodes, and patients. 


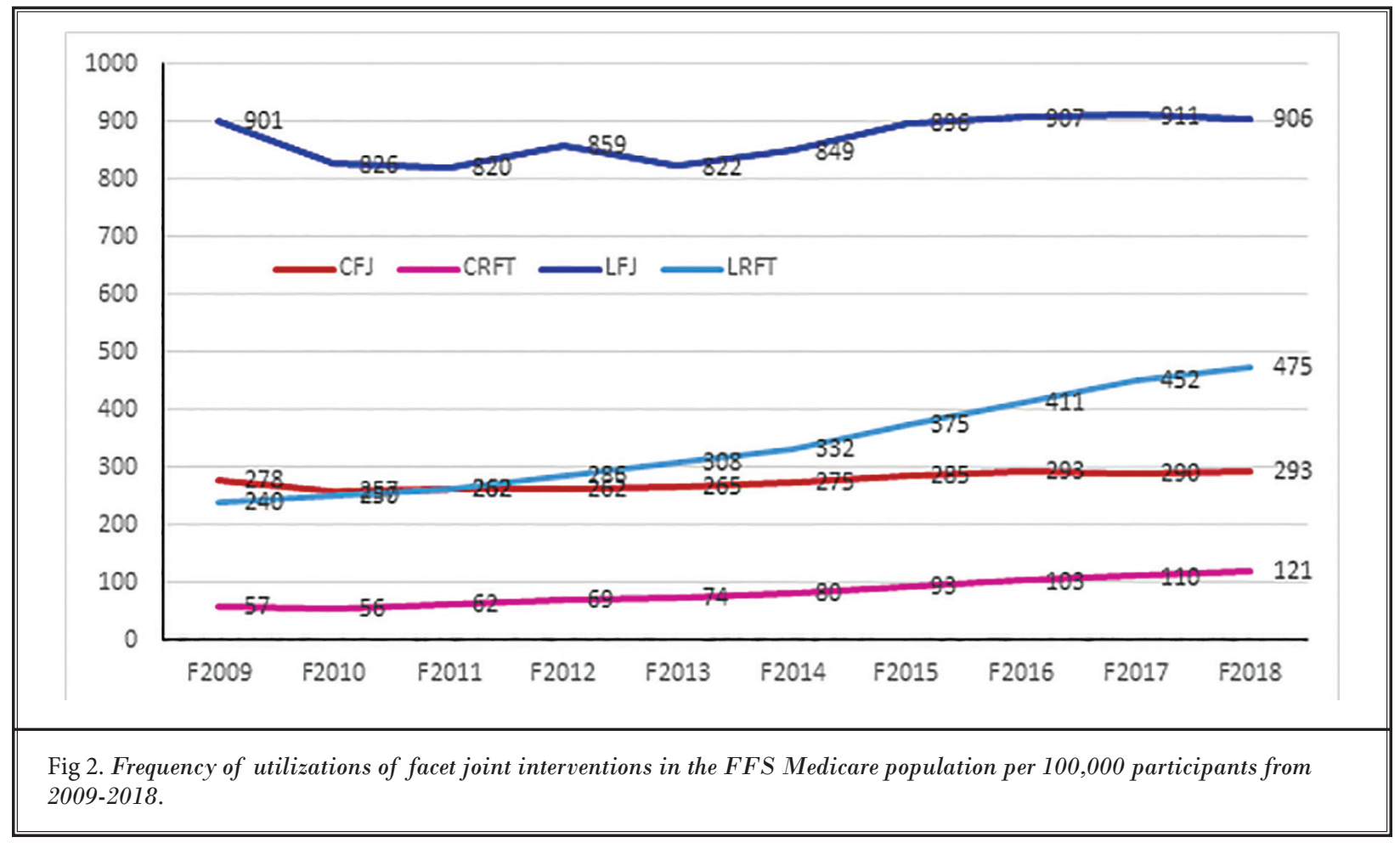

visit in 2009 was $\$ 813.47$ which increased to $\$ 849.86$ in 2018. However, inflation-adjusted allowed charges per visit increased the charges to $\$ 957.16$ in 2009 to $\$ 849.86$ in 2018 , an $11 \%$ overall decrease or annual decrease of $1.3 \%$.

Table 7 shows total allowed charges by place of service and type of the procedure. There was a significant increase in radiofrequency neurotomy procedures in all settings than for facet joint injections. As shown in other tables, inflation-adjusted allowed charges per service increased $6 \%$ with an annual increase of $0.7 \%$ compared to overall increase of $25 \%$ with an annual increase of $2.5 \%$.

\section{Specialty Characteristics}

Appendix Table 1 shows utilization of facet joint interventions by episodes by specialty. The majority of the procedures were performed by physicians who considered themselves interventional pain management, including anesthesiology with CMS designations of -05 , -09 , and -72 . In 2009 , this group constituted $63 \%$ of the procedures, whereas in 2018, it increased to $73 \%$. Physical medicine and rehabilitation, with a designation of -25 , revealed significant increased utilization from $15 \%$ in 2009 to $20 \%$ in 2018; however, neurology, with the designation of -13 , showed a significant decline from $4 \%$ in 2009 to $1 \%$ in 2018 . Interventional radiologists showed increased utilization, whereas diagnostic radiologists demonstrated a decline with an overall significant net decrease in utilization. All other specialties, including family practitioners and other providers, declined significantly.

\section{State Wise Distribution Characteristics}

Appendix Table 2 shows the utilization of facet joint interventions based by Medicare carrier and state from 2016. Noridian, the largest and most aggressive carrier regarding the development of specific interventional policies to be utilized across the nation to reduce utilization patterns, showed an overall increase of $1.7 \%$ per year and $16.1 \%$ from 2009 to 2018. Palmetto GBA with liberal policies in reference to the cutoff of threshold for positive blocks reducing it from $80 \%$ to $50 \%$ has shown significant increases compared to other states with a total of $43.9 \%$ from 2009 to 2018 with an annual increase of $4.1 \%$. CGS utilizing prior policies, which were in contrast to policies by Novitas, showed an overall increase of $37.7 \%$, with an annual increase of $3.6 \%$. Overall, utilization was high with similar 
Trends of Expenditures of Facet Joint Interventions in Medicare Population

\begin{tabular}{|c|c|c|c|c|c|c|c|c|c|c|c|c|c|c|c|c|c|c|c|c|c|c|c|c|c|c|c|}
\hline & 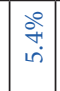 & 竞 & 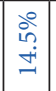 & 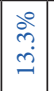 & \begin{tabular}{|c|}
$\circ$ \\
$\infty$ \\
$\infty$ \\
$\infty$
\end{tabular} & & $\begin{array}{l}0 \\
\text { mे } \\
i n\end{array}$ & \begin{tabular}{|l|}
$\stackrel{0}{\circ}$ \\
$\dot{m}$
\end{tabular} & 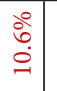 & $\stackrel{\circ}{\circ}$ & 通 & वें & ํํㄱ & $\begin{array}{l}\stackrel{\circ}{\infty} \\
\stackrel{\Xi}{=}\end{array}$ & 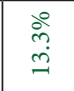 & \begin{tabular}{|l|} 
\\
$\infty$ \\
$\infty$ \\
+ \\
+
\end{tabular} & & $\begin{array}{ll}\circ \\
\text { in } \\
\text { ñ }\end{array}$ & 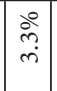 & \begin{tabular}{|c|}
$\stackrel{\circ}{\circ}$ \\
$\stackrel{\text { जe }}{\mathrm{j}}$
\end{tabular} & \begin{tabular}{|l|}
$\stackrel{\circ}{\circ}$ \\
$\stackrel{\Xi}{\exists}$
\end{tabular} & 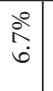 & के & 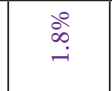 & $\stackrel{\circ}{\circ}$ & 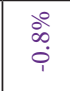 & 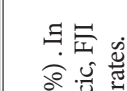 \\
\hline & $\frac{2}{b}$ & $\begin{array}{l}0 \\
\text { in } \\
\end{array}$ & \begin{tabular}{|l|}
$\stackrel{0}{0}$ \\
$\stackrel{\sim}{\sim}$
\end{tabular} & \begin{tabular}{l|}
0 \\
$\stackrel{े}{0}$ \\
$\stackrel{े}{0}$
\end{tabular} & $\begin{array}{l}\stackrel{0}{\circ} \\
\stackrel{2}{=}\end{array}$ & & in & \begin{tabular}{|l|}
$\stackrel{\circ}{\not}$ \\
\end{tabular} & 号 & $\stackrel{\circ}{\stackrel{\circ}{a}}$ & $\stackrel{\infty}{\infty}$ & ळे & $\stackrel{\circ}{\ni}$ & $\stackrel{\circ}{\stackrel{\circ}{\beth}}$ & 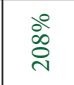 & 仓ें & & 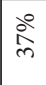 & iñ & \begin{tabular}{c|}
$\circ$ \\
$\vdots$ \\
$\infty$ \\
\hdashline
\end{tabular} & \begin{tabular}{|l|}
\multirow{2}{0}{} \\
ô \\
\end{tabular} & ฉे & iे & $\stackrel{\infty}{\infty}$ & $\stackrel{\circ}{\infty}$ & مें & 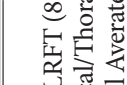 \\
\hline 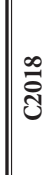 & \begin{tabular}{|c|}
$\hat{a}$ \\
0 \\
0 \\
$\vec{a}$ \\
0 \\
0 \\
0 \\
0
\end{tabular} & 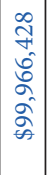 & \begin{tabular}{|c|} 
\\
$\infty$ \\
$\infty$ \\
0 \\
0 \\
$\infty$ \\
$\infty$ \\
$\infty$ \\
$\infty$
\end{tabular} & 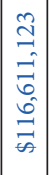 & 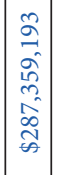 & & 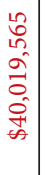 & 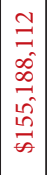 & 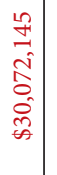 & $\begin{array}{l}8 \\
0 \\
0 \\
0 \\
0 \\
b \\
0 \\
\infty\end{array}$ & 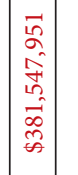 & $\begin{array}{l}m \\
m \\
\infty \\
\infty \\
\infty \\
+ \\
\infty \\
\infty \\
\infty\end{array}$ & $\begin{array}{l}\alpha \\
0 \\
\alpha \\
\sigma \\
\sigma \\
\sigma \\
\sigma \\
\sigma\end{array}$ & 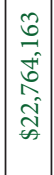 & 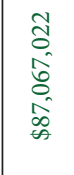 & 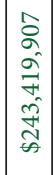 & $\begin{array}{l}\infty \\
\stackrel{\tilde{U}}{0} \\
\end{array}$ & 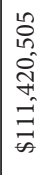 & 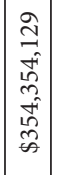 & 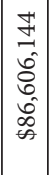 & 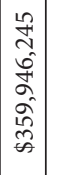 & $\begin{array}{l}\vec{b} \\
0 \\
\hat{0} \\
\hat{n} \\
\hat{a} \\
\hat{\omega}\end{array}$ & 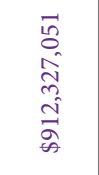 & $\begin{array}{l}0 \\
h \\
0 \\
0 \\
0 \\
\substack{n \\
\infty}\end{array}$ & $\frac{n}{6}$ & $\begin{array}{l}\stackrel{\infty}{\infty} \\
\stackrel{-}{-\infty}\end{array}$ & 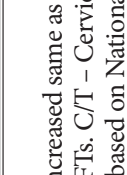 \\
\hline 宊 & 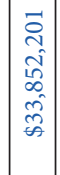 & 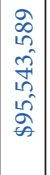 & 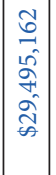 & 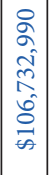 & 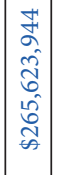 & & 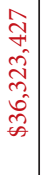 & 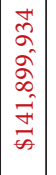 & 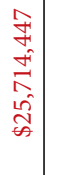 & 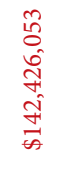 & 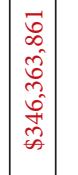 & $\begin{array}{ll}1 \\
\infty \\
\infty \\
0 \\
0 \\
\infty \\
\infty \\
\infty \\
\infty \\
\infty\end{array}$ & $\begin{array}{l}\vec{n} \\
2 \\
0 \\
0 \\
0 \\
2 \\
\vdots \\
\infty\end{array}$ & $\begin{array}{l}\overrightarrow{\hat{v}} \\
\hat{\sigma} \\
\hat{\sigma} \\
\hat{\sigma} \\
\vec{\omega}\end{array}$ & 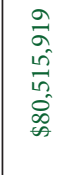 & 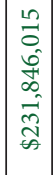 & ปิ & 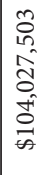 & 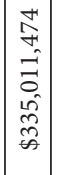 & 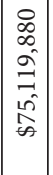 & 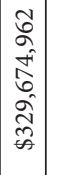 & $\begin{array}{c}\stackrel{2}{1} \\
\infty \\
\hat{N} \\
\infty \\
0 \\
\infty \\
\infty \\
\infty \\
\infty\end{array}$ & 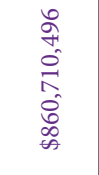 & 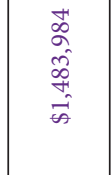 & $\stackrel{n}{\infty}$ & $\begin{array}{l}\stackrel{2}{\hat{n}} \\
\stackrel{-\infty}{\infty}\end{array}$ & 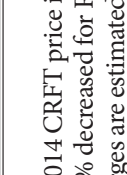 \\
\hline |ั & \begin{tabular}{|l|}
$\hat{n}$ \\
$\hat{2}$ \\
$\hat{\sigma}$ \\
0 \\
0 \\
$\hat{n}$ \\
$\hat{n}$ \\
\end{tabular} & 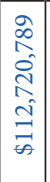 & 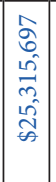 & 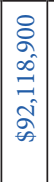 & 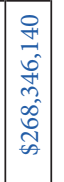 & & 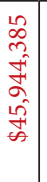 & 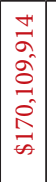 & 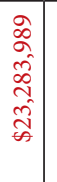 & \begin{tabular}{l}
$\infty$ \\
$\infty$ \\
$\infty$ \\
0 \\
$\infty$ \\
$\infty$ \\
\multirow{J}{*}{} \\
$\vec{\infty}$
\end{tabular} & 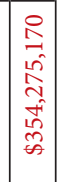 & \begin{tabular}{|l|}
$\infty$ \\
0 \\
0 \\
0 \\
0 \\
0 \\
$\infty$ \\
$\infty$ \\
$\infty$ \\
$\infty$
\end{tabular} & 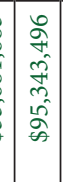 & 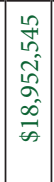 & 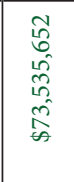 & 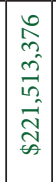 & 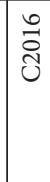 & 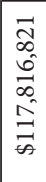 & 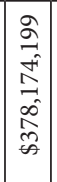 & 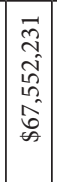 & 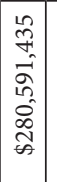 & 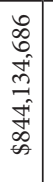 & 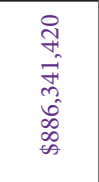 & 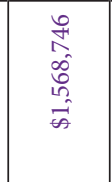 & $\vec{b}$ & $\begin{array}{l}\overrightarrow{\vec{\sigma}} \\
\overrightarrow{\vec{s}}\end{array}$ & 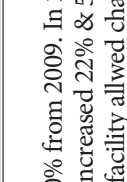 \\
\hline 颉 & $\left|\begin{array}{c}0 \\
0 \\
0 \\
0 \\
0 \\
0 \\
i \\
0 \\
0 \\
0\end{array}\right|$ & $\begin{array}{c}0 \\
\hat{N} \\
\hat{n} \\
\infty \\
\infty \\
\infty \\
\infty \\
\infty \\
\infty \\
\infty\end{array}$ & 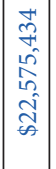 & 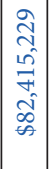 & 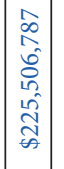 & & 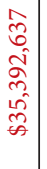 & 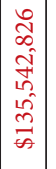 & 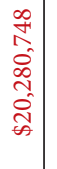 & 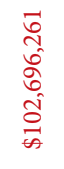 & 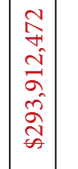 & $\begin{array}{l}2 \\
0 \\
0 \\
+1 \\
-1 \\
-3 \\
\infty \\
\infty\end{array}$ & $\mid \begin{array}{l}2 \\
2 \\
0 \\
0 \\
\vdots \\
0 \\
0 \\
0\end{array}$ & $\begin{array}{l}\overrightarrow{\hat{f}} \\
\infty \\
0 \\
0 \\
\hat{\vec{t}} \\
\omega\end{array}$ & 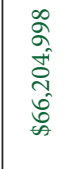 & 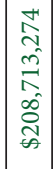 & 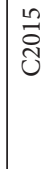 & 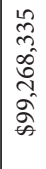 & 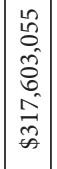 & 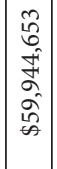 & 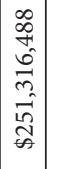 & 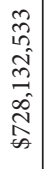 & 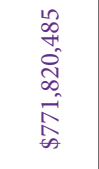 & $\begin{array}{l}8 \\
\infty \\
\infty \\
0 \\
\stackrel{0}{1} \\
\rightarrow\end{array}$ & $\underset{\Leftrightarrow}{H}$ & $\begin{array}{l}\infty \\
\stackrel{\infty}{-1} \\
\stackrel{\rightarrow}{A}\end{array}$ & 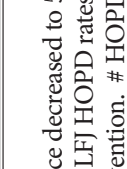 \\
\hline | & 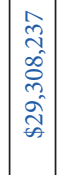 & $\left|\begin{array}{c}0 \\
0 \\
0 \\
\infty \\
0 \\
12 \\
\hat{1} \\
\infty \\
\infty\end{array}\right|$ & 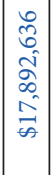 & 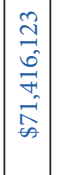 & $\mid$\begin{tabular}{c|}
0 \\
0 \\
0 \\
$\omega$ \\
0 \\
0 \\
0 \\
2 \\
2
\end{tabular} & & 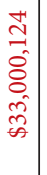 & 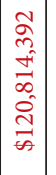 & 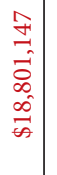 & 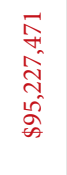 & 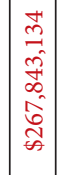 & $\begin{array}{l}\infty \\
0 \\
0 \\
\tilde{d} \\
m \\
0 \\
0 \\
0 \\
0\end{array}$ & 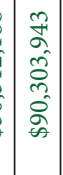 & 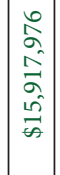 & 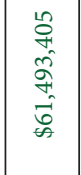 & 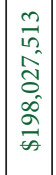 & 节 & 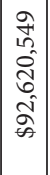 & 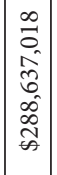 & 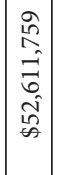 & 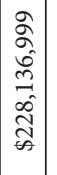 & $\left|\begin{array}{c}1 \\
\hat{3} \\
0 \\
0 \\
0 \\
\hat{d} \\
b \\
0 \\
0\end{array}\right|$ & $\begin{array}{l}\hat{2} \\
\hat{R} \\
0 \\
\hat{N} \\
\hat{0} \\
0 \\
0\end{array}$ & 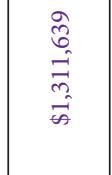 & $\stackrel{m}{\infty}$ & $\underset{\stackrel{N}{N}}{\stackrel{N}{A}}$ & 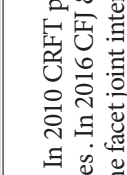 \\
\hline 节 & 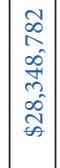 & 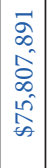 & $\left|\begin{array}{c}0 \\
\infty \\
\infty \\
0 \\
\hat{N} \\
0 \\
0 \\
\infty \\
\infty\end{array}\right|$ & $\left|\begin{array}{c|}\infty \\
\infty \\
0 \\
0 \\
\infty \\
\infty \\
\infty \\
b \\
\infty \\
\infty\end{array}\right|$ & 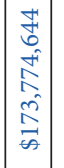 & & 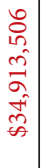 & 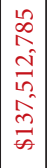 & 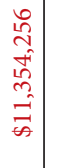 & $\begin{array}{l}\overrightarrow{5} \\
= \\
0 \\
0 \\
\hat{0} \\
\hat{n}\end{array}$ & $\mid \begin{array}{l}0 \\
\hat{1} \\
0 \\
0 \\
0 \\
0 \\
0 \\
0 \\
\infty\end{array}$ & 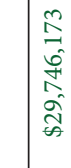 & $\begin{array}{l}\overrightarrow{2} \\
0 \\
0 \\
-1 \\
0 \\
0 \\
\infty \\
\infty\end{array}$ & 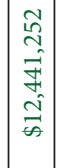 & $\begin{array}{l}\hat{\alpha} \\
\hat{n} \\
\hat{n} \\
\hat{n} \\
\hat{n}\end{array}$ & 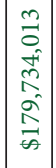 & 荇 & 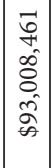 & 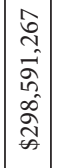 & 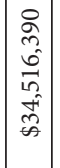 & 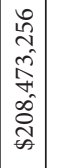 & 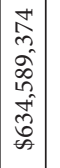 & $\begin{array}{l}\text { त } \\
\text { N } \\
0 \\
0 \\
0 \\
0 \\
0 \\
0 \\
0 \\
0\end{array}$ & $\begin{array}{l}m \\
\hat{n} \\
\hat{n} \\
\hat{n} \\
\Leftrightarrow \\
\Leftrightarrow\end{array}$ & $\stackrel{m}{\Leftrightarrow}$ & $\begin{array}{l}\infty \\
\infty \\
\infty \\
\rightarrow \\
\vec{\infty}\end{array}$ & 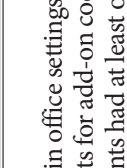 \\
\hline 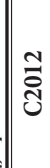 & 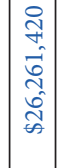 & 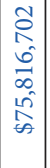 & 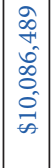 & 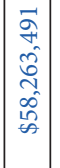 & 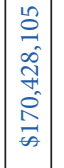 & & 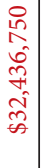 & 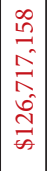 & 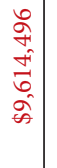 & $\begin{array}{l}m \\
\infty \\
\infty \\
+\hat{N} \\
+ \\
0 \\
0 \\
0\end{array}$ & 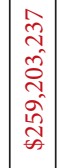 & $\begin{array}{l}\infty \\
\infty \\
\infty \\
0 \\
\infty \\
0 \\
\hat{N} \\
\infty \\
\infty\end{array}$ & $\begin{array}{l}\infty \\
\infty \\
0 \\
0 \\
\alpha \\
0 \\
\infty \\
\infty\end{array}$ & $\left|\begin{array}{l}0 \\
\vec{z} \\
\hat{d} \\
\delta \\
\hat{d} \\
\omega\end{array}\right|$ & 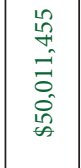 & 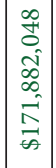 & ปี & 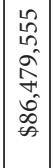 & 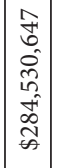 & 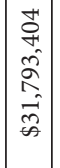 & 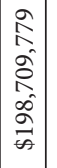 & $\left|\begin{array}{c}2 \\
2 \\
0 \\
0 \\
0 \\
0 \\
0 \\
0 \\
0 \\
0 \\
0\end{array}\right|$ & 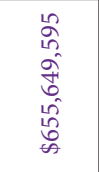 & 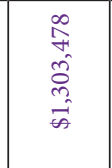 & $\frac{m}{\infty}$ & $\begin{array}{l}\vec{F} \\
\vec{\infty} \\
\vec{H}\end{array}$ & 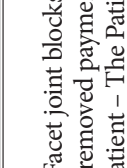 \\
\hline ปั่ & 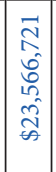 & 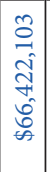 & \begin{tabular}{|c|} 
\\
$\hat{\alpha}$ \\
$\hat{d}$ \\
$\alpha$ \\
$\infty$ \\
$\infty$ \\
$\infty$
\end{tabular} & 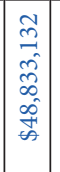 & 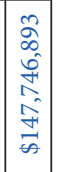 & & 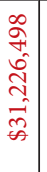 & 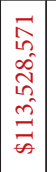 & 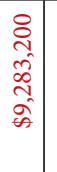 & $\begin{array}{l}\text { से } \\
\text { مे } \\
\overrightarrow{0} \\
0 \\
0 \\
0 \\
0 \\
0\end{array}$ & 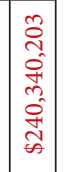 & \begin{tabular}{|l}
0 \\
$\hat{0}$ \\
$\infty$ \\
$\infty$ \\
$\infty$ \\
$\hat{N}$ \\
$\infty$
\end{tabular} & 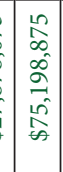 & 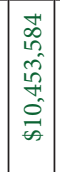 & 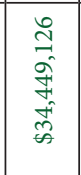 & 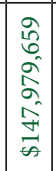 & تิ & 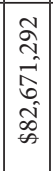 & 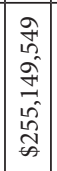 & 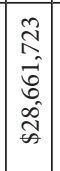 & 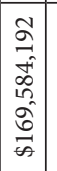 & 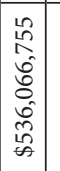 & 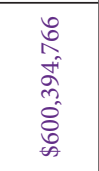 & 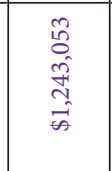 & $\tilde{\infty}$ & 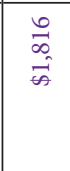 & 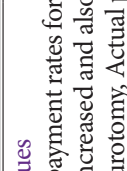 \\
\hline ปั่ & 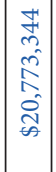 & 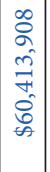 & 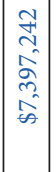 & 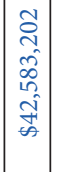 & 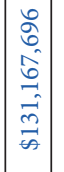 & & 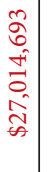 & $\mid \begin{array}{c}0 \\
0 \\
0 \\
0 \\
0 \\
0 \\
0 \\
0 \\
\infty \\
\infty\end{array}$ & 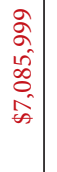 & $\begin{array}{l}\sqrt{0} \\
0 \\
0 \\
0 \\
0 \\
0 \\
0 \\
0\end{array}$ & 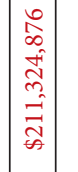 & 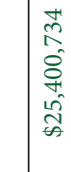 & $\begin{array}{l}0 \\
0 \\
0 \\
0 \\
0 \\
0 \\
0 \\
0 \\
\infty\end{array}$ & 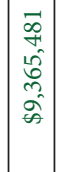 & $\begin{array}{l}\infty \\
\infty \\
\infty \\
\infty \\
\infty \\
\infty \\
0 \\
0 \\
\infty\end{array}$ & 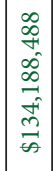 & 吾 & 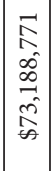 & 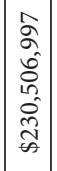 & 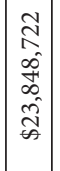 & 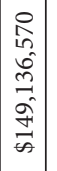 & 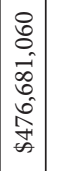 & 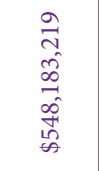 & $\begin{array}{l}\infty \\
\infty \\
0 \\
0 \\
0 \\
-1 \\
-1\end{array}$ & $\stackrel{\approx}{*}$ & $\begin{array}{l}\stackrel{N}{N} \\
\stackrel{\infty}{\infty}\end{array}$ & 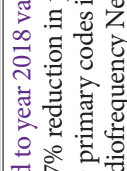 \\
\hline ठે & 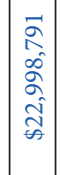 & 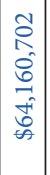 & 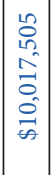 & 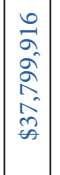 & 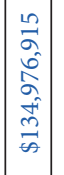 & & 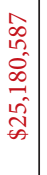 & 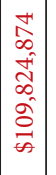 & 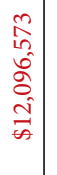 & $\begin{array}{l}0 \\
0 \\
0 \\
0 \\
0 \\
0 \\
0 \\
0\end{array}$ & 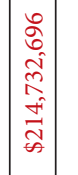 & 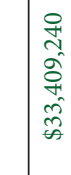 & $\begin{array}{c}0 \\
0 \\
0 \\
0 \\
0 \\
\alpha \\
0 \\
\infty \\
\infty\end{array}$ & 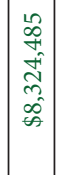 & 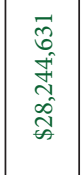 & 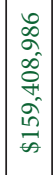 & ठे̀ & \begin{tabular}{|c|}
$\infty$ \\
0 \\
0 \\
$\infty$ \\
$\infty$ \\
0 \\
$\infty$ \\
$\infty$ \\
$\infty$ \\
0 \\
0
\end{tabular} & 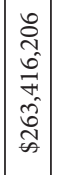 & 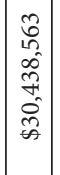 & 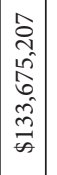 & 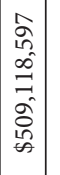 & $\begin{array}{l}\infty \\
\infty \\
0 \\
\infty \\
0 \\
0 \\
0 \\
0 \\
0 \\
0\end{array}$ & $\begin{array}{l}\infty \\
\infty \\
0 \\
0 \\
0 \\
\infty \\
\Rightarrow \\
\infty\end{array}$ & $\vec{m}$ & $\begin{array}{l}\stackrel{\mu}{2} \\
\stackrel{\omega}{\omega}\end{array}$ & 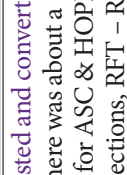 \\
\hline$\frac{3}{4}$ & 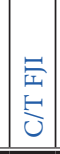 & & 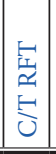 & 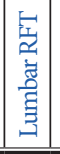 & 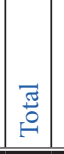 & 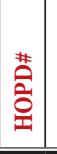 & $\begin{array}{l}\overrightarrow{4} \\
\stackrel{5}{5} \\
\end{array}$ & 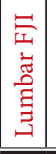 & 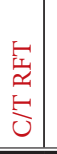 & 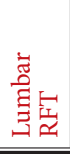 & 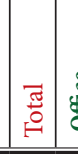 & 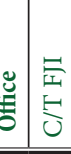 & 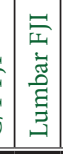 & 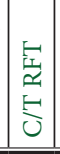 & 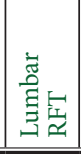 & \begin{tabular}{|l|}
\multirow{\pi}{*}{} \\
$\stackrel{0}{0}$ \\
\end{tabular} & 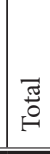 & 空 & 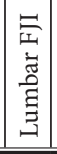 & 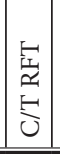 & 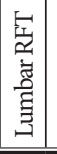 & 胥 & 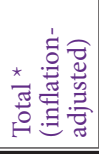 & 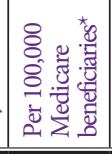 & 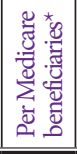 & 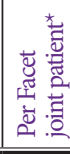 & 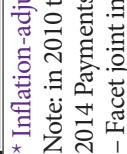 \\
\hline
\end{tabular}




\begin{tabular}{|c|c|c|c|c|c|c|c|c|c|c|c|c|c|c|c|c|c|c|c|c|c|c|c|c|c|c|}
\hline હ્ડ & 亏ें & $\mid \begin{array}{c} \\
\stackrel{+}{\circ} \\
\end{array}$ & 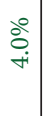 & خें & & & $\begin{array}{l}\stackrel{0}{\circ} \\
\vec{i}\end{array}$ & خેّ & ণे & 今े & & & 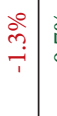 & ڤ̊ํำ & $\begin{array}{c}\text { oें } \\
\text { iे } \\
i\end{array}$ & & ஓें & वे & | & 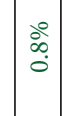 & & 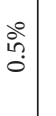 & & $\stackrel{\circ}{\stackrel{9}{\rightarrow}}$ & & 离 \\
\hline 表 & $\stackrel{\circ}{\stackrel{\circ}{\beth}}$ & $\stackrel{\circ}{\circ}$ & ঐ̊ำ & ते & & & ڤें & ڤે̀ & 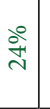 & $\begin{array}{c}\stackrel{\circ}{\circ} \\
\stackrel{\sim}{0}\end{array}$ & & ڤँ๊ & $\stackrel{\circ}{\overrightarrow{7}}$ & ఫ̊ & ڤे̀ & & अें & $\stackrel{0}{\circ}$ & ?ִ & ฉ̊ & & $\stackrel{\leftrightarrow}{\not}$ & & $\stackrel{\circ}{\frac{2}{7}}$ & & \\
\hline 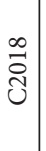 & 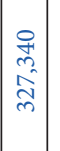 & 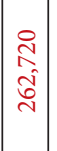 & 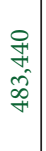 & 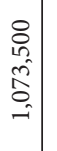 & & & 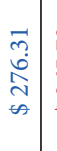 & 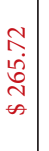 & 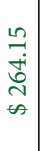 & $\mid \begin{array}{c}\overrightarrow{1} \\
0 \\
\infty \\
0 \\
\sim \\
\infty\end{array}$ & & $\begin{array}{l}n \\
\hat{n} \\
\dot{0} \\
0 \\
\infty\end{array}$ & 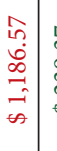 & $\begin{array}{l}\hat{m} \\
\grave{j} \\
\hat{i} \\
\hat{\infty}\end{array}$ & $\begin{array}{l}\tilde{S} \\
\dot{D} \\
0 \\
0 \\
n\end{array} \mid$ & & & خે & 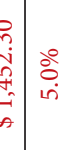 & 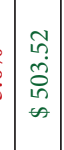 & 今े & \begin{tabular}{c|c}
0 \\
$\infty$ \\
$\dot{\hat{q}}$ \\
$\infty$ \\
$\infty$ \\
$\leftrightarrow$
\end{tabular} & ì & $\begin{array}{l}\infty \\
\infty \\
\infty \\
\dot{\alpha} \\
\infty \\
\Leftrightarrow \\
\Leftrightarrow\end{array}$ & & $\overrightarrow{\tilde{N}}$ \\
\hline 氕 & 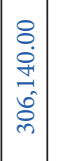 & 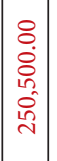 & 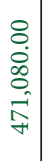 & 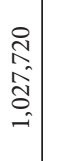 & & & 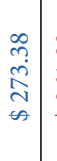 & 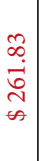 & 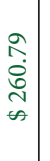 & $\left|\begin{array}{c}2 \\
\stackrel{i}{i} \\
i \\
\infty \\
\infty\end{array}\right|$ & & 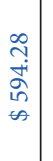 & 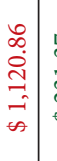 & $\begin{array}{l}\stackrel{a}{m} \\
\underset{\sim}{\sim} \\
\Leftrightarrow\end{array}$ & 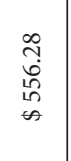 & & & مَّ & 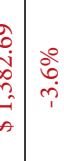 & 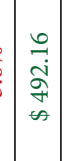 & |خ & $\begin{array}{l}\hat{O} \\
\dot{\vec{d}} \\
\infty \\
\infty \\
\hat{A}\end{array}$ & $\begin{array}{l}\stackrel{\circ}{\circ} \\
\stackrel{+}{+}\end{array}$ & 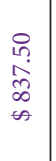 & & \\
\hline $\begin{array}{l}0 \\
\stackrel{0}{0} \\
\text { Uิ }\end{array}$ & $\left|\begin{array}{l}0 \\
0 \\
o \\
0 \\
\sim \\
\sim \\
\sim\end{array}\right|$ & 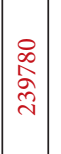 & 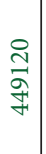 & $\begin{array}{l}\stackrel{0}{ } \\
\hat{N} \\
\hat{\alpha}\end{array}$ & & & $\begin{array}{l}\mathcal{y} \\
\text { i } \\
\text { J } \\
\Leftrightarrow\end{array}$ & 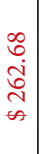 & 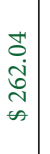 & 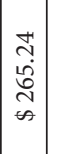 & & $\begin{array}{l}\vec{\infty} \\
0 \\
0 \\
0 \\
\infty\end{array}$ & 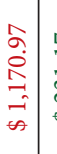 & 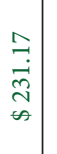 & 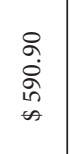 & & & 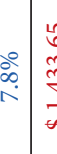 & 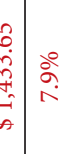 & 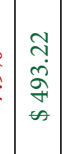 & $\stackrel{\circ}{\stackrel{\circ}{-}}$ & 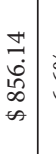 & فㅇํㅇ & $\begin{array}{l}\Delta \\
0 \\
\infty \\
\infty \\
\infty \\
\infty\end{array}$ & & \\
\hline 号 & $\left|\begin{array}{l}8 \\
\vdots \\
2 \\
\hat{2} \\
\\
\mid\end{array}\right|$ & 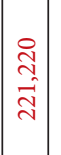 & 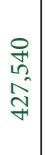 & 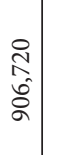 & & & $\begin{array}{c}\vec{m} \\
\vdots \\
\vdots \\
i \\
\infty\end{array}$ & $\begin{array}{l}\hat{\imath} \\
\infty \\
\infty \\
\hat{\jmath} \\
\infty\end{array}$ & 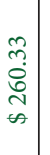 & $\left|\begin{array}{c}\hat{\imath} \\
\hat{d} \\
\hat{\sigma} \\
\infty\end{array}\right|$ & & $\begin{array}{l}\infty \\
\infty \\
\infty \\
0 \\
0 \\
0 \\
\infty\end{array}$ & \begin{tabular}{c|c}
$\vec{\alpha}$ \\
$\overrightarrow{0}$ \\
$\vec{b}$ \\
$\vec{\theta}$
\end{tabular} & 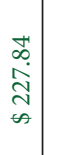 & $\begin{array}{l}n \\
n \\
0 \\
0 \\
0 \\
n \\
\infty\end{array} \mid$ & & & لَّ & 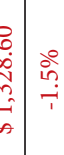 & 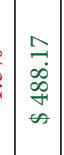 & 今े & 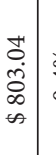 & $\begin{array}{l}\text { के } \\
\text { के }\end{array}$ & $\begin{array}{l}\text { तै } \\
\underset{B}{\infty} \\
\infty \\
\infty \\
\infty\end{array}$ & & $\bar{\Sigma}$ \\
\hline $\begin{array}{l}\stackrel{+}{\vec{S}} \\
\text { तु }\end{array}$ & 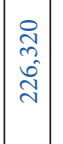 & 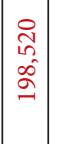 & 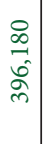 & 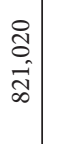 & & & $\begin{array}{l}f \\
+1 \\
0 \\
0 \\
0 \\
s\end{array}$ & $\begin{array}{l}7 \\
+ \\
\infty \\
\infty \\
\sim \\
\infty\end{array}$ & 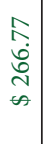 & $\left|\begin{array}{c}\vec{i} \\
\hat{b} \\
i \\
\infty \\
\infty\end{array}\right|$ & & $\begin{array}{l}\tilde{c} \\
\infty \\
\infty \\
0 \\
\infty \\
\Leftrightarrow\end{array}$ & 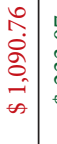 & 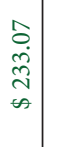 & 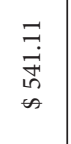 & & & | & 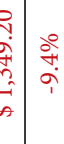 & 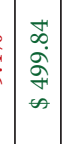 & 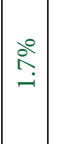 & 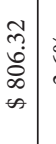 & 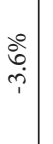 & 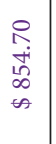 & 它 & \\
\hline $\begin{array}{l}\text { ma } \\
\text { तु }\end{array}$ & 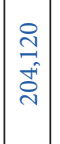 & $\mid \begin{array}{l}0 \\
0 \\
\infty \\
\infty \\
\infty \\
-1\end{array}$ & 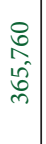 & 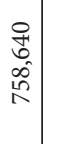 & & & 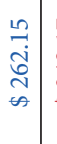 & 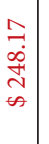 & 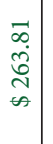 & 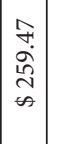 & & $\begin{array}{l}\stackrel{\vartheta}{\overrightarrow{2}} \\
0 \\
\infty \\
\infty \\
\Leftrightarrow\end{array}$ & 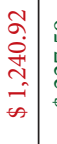 & 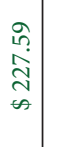 & $\begin{array}{l}\overrightarrow{0} \\
i \\
i n \\
i n \\
\theta\end{array}$ & & & 党 & 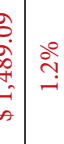 & 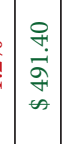 & 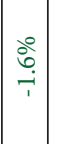 & 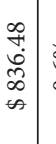 & & 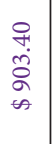 & ְִ & \\
\hline 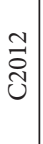 & 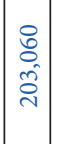 & 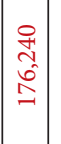 & 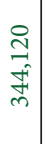 & 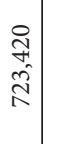 & & & \begin{tabular}{l}
7 \\
\multirow{+}{*}{} \\
d. \\
$\Leftrightarrow$
\end{tabular} & 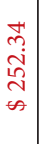 & 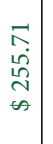 & 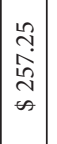 & & 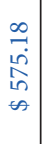 & 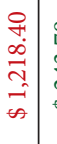 & 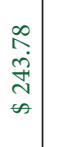 & $\begin{array}{c}\vec{y} \\
\underset{+}{+} \\
i n \\
\infty \\
\infty\end{array}$ & & 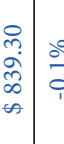 & 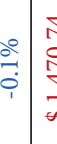 & 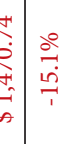 & 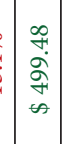 & $\stackrel{\circ}{\circ}$ & 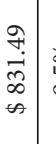 & ڤั่ & 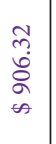 & 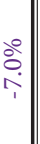 & \\
\hline 节 & 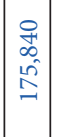 & 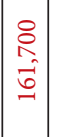 & 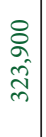 & 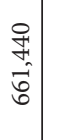 & & & 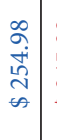 & 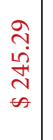 & 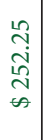 & $\left|\begin{array}{c}\hat{i} \\
\vec{i} \\
i \\
\infty \\
\infty\end{array}\right|$ & & $\begin{array}{l}n \\
0 \\
0 \\
0 \\
\infty \\
n \\
\infty\end{array}$ & 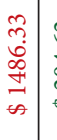 & 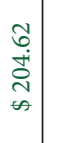 & $\begin{array}{l}7 \\
0 \\
0 \\
0 \\
\infty\end{array}$ & & 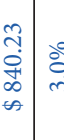 & 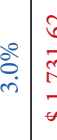 & 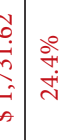 & $\mid \begin{array}{l}\infty \\
\infty \\
b \\
b \\
b \\
w \\
\omega\end{array}$ & $\begin{array}{l}\text { ڤे } \\
\text { aे }\end{array}$ & 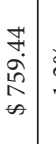 & ஸें & 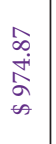 & 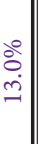 & \\
\hline 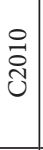 & $\left|\begin{array}{l}0 \\
\stackrel{0}{0} \\
0 \\
0 \\
-1\end{array}\right|$ & $\mid \begin{array}{l}0 \\
0 \\
-1 \\
-10 \\
-1\end{array}$ & $\begin{array}{l}\text { בे } \\
\hat{i} \\
\text { }\end{array}$ & 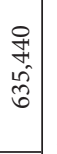 & & & 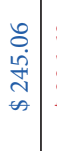 & 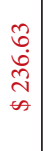 & 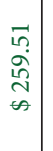 & 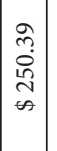 & & $\begin{array}{l}0 \\
\infty \\
0 \\
0 \\
i \\
\infty \\
\infty\end{array}$ & 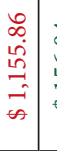 & 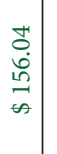 & $\begin{array}{l}\hat{a} \\
\hat{a} \\
\stackrel{+}{\infty}\end{array}$ & & 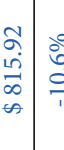 & |. & 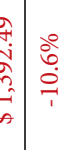 & 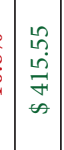 & $\underset{\stackrel{\circ}{\circ}}{\stackrel{\circ}{=}}$ & 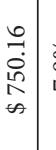 & ळ. & $\begin{array}{l}\infty \\
0 \\
\mathbb{j} \\
\infty \\
\infty \\
\infty\end{array}$ & $亠^{\circ}$ & \\
\hline 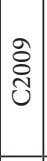 & 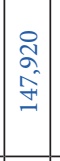 & 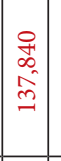 & $\begin{array}{l}8 \\
\vdots \\
\vdots \\
\vdots \\
0\end{array}$ & $\begin{array}{l}8 \\
0 \\
0 \\
1 \\
0 \\
0\end{array}$ & 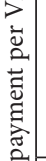 & & 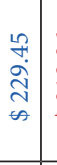 & \begin{tabular}{l}
$\stackrel{0}{0}$ \\
$\infty$ \\
$\vec{\sim}$ \\
\multirow{2}{*}{}
\end{tabular} & 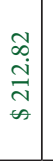 & 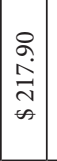 & & $\begin{array}{l}0 \\
0 \\
0 \\
0 \\
0 \\
0 \\
\infty\end{array}$ & 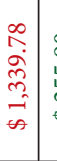 & 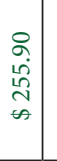 & $\begin{array}{l}\hat{n} \\
\hat{\alpha} \\
\hat{n} \\
n \\
n\end{array}$ & 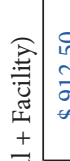 & $\begin{array}{l}\text { in } \\
\text { in } \\
\text { In }\end{array}$ & $\forall$ & 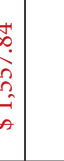 & 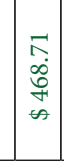 & & 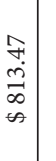 & & 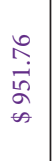 & & \\
\hline 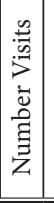 & \begin{tabular}{|l|}
0 \\
0 \\
\end{tabular} & \begin{tabular}{|l}
0 \\
0 \\
0 \\
0 \\
出
\end{tabular} & 10 & 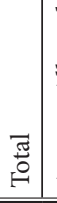 & $\frac{\tilde{\Delta}}{4}$ & 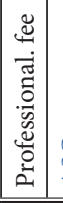 & $\begin{array}{l}0 \\
\text { Zे }\end{array}$ & 登 & $\begin{array}{l}\text { : } \\
0\end{array}$ & 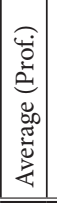 & $\stackrel{\Xi}{\square}$ & 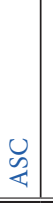 & 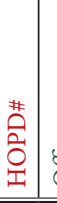 & $\begin{array}{l}\stackrel{\mathscr{E}}{0} \\
\end{array}$ & 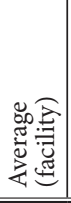 & 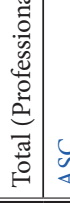 & 仓ิ & \begin{tabular}{c|c}
0 \\
0 \\
0
\end{tabular} & 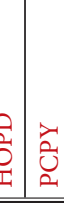 & 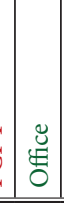 & \begin{tabular}{|l|} 
\\
U. \\
|
\end{tabular} & & స్తీ & 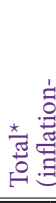 & & \\
\hline
\end{tabular}


Trends of Expenditures of Facet Joint Interventions in Medicare Population

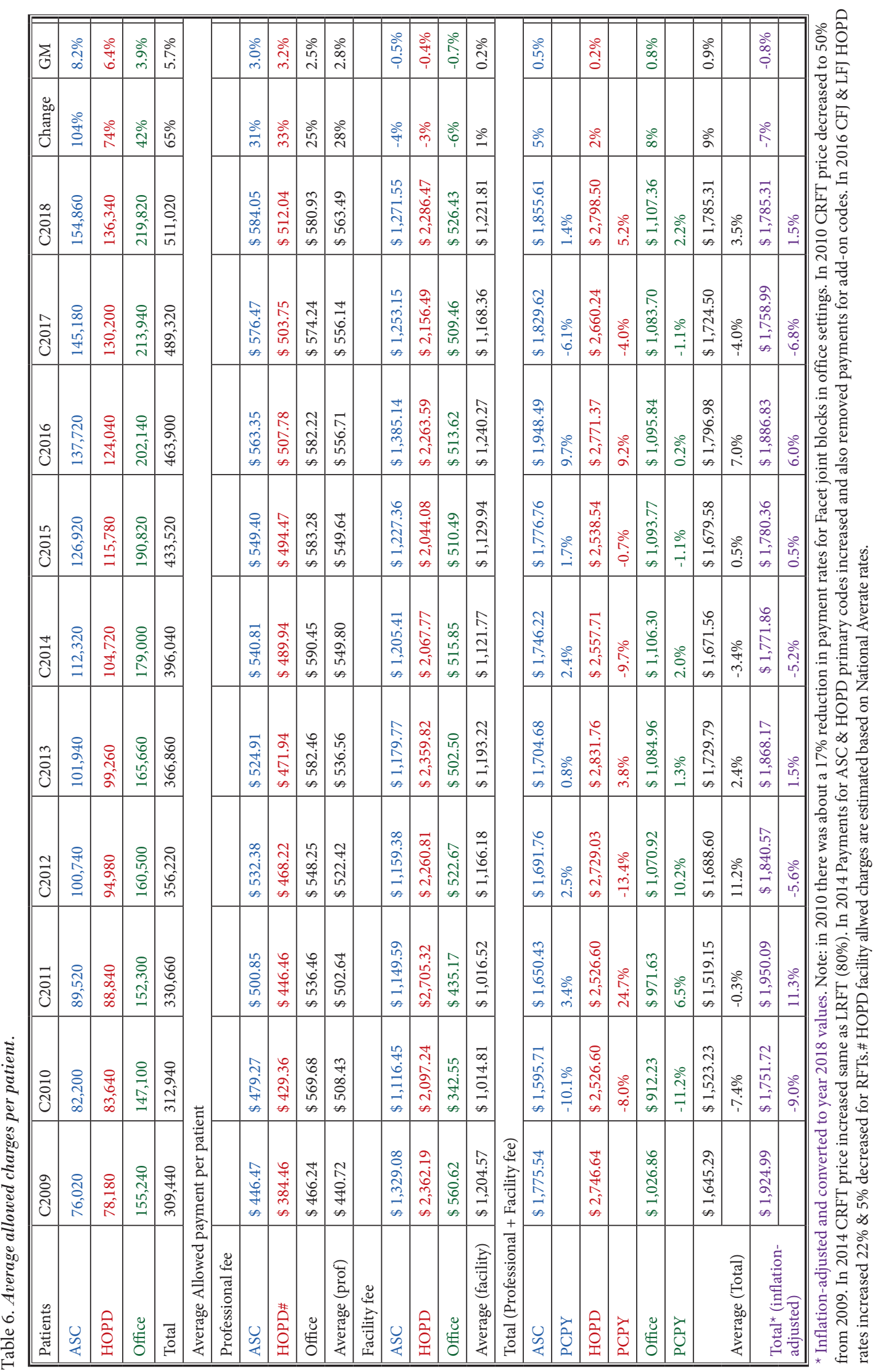




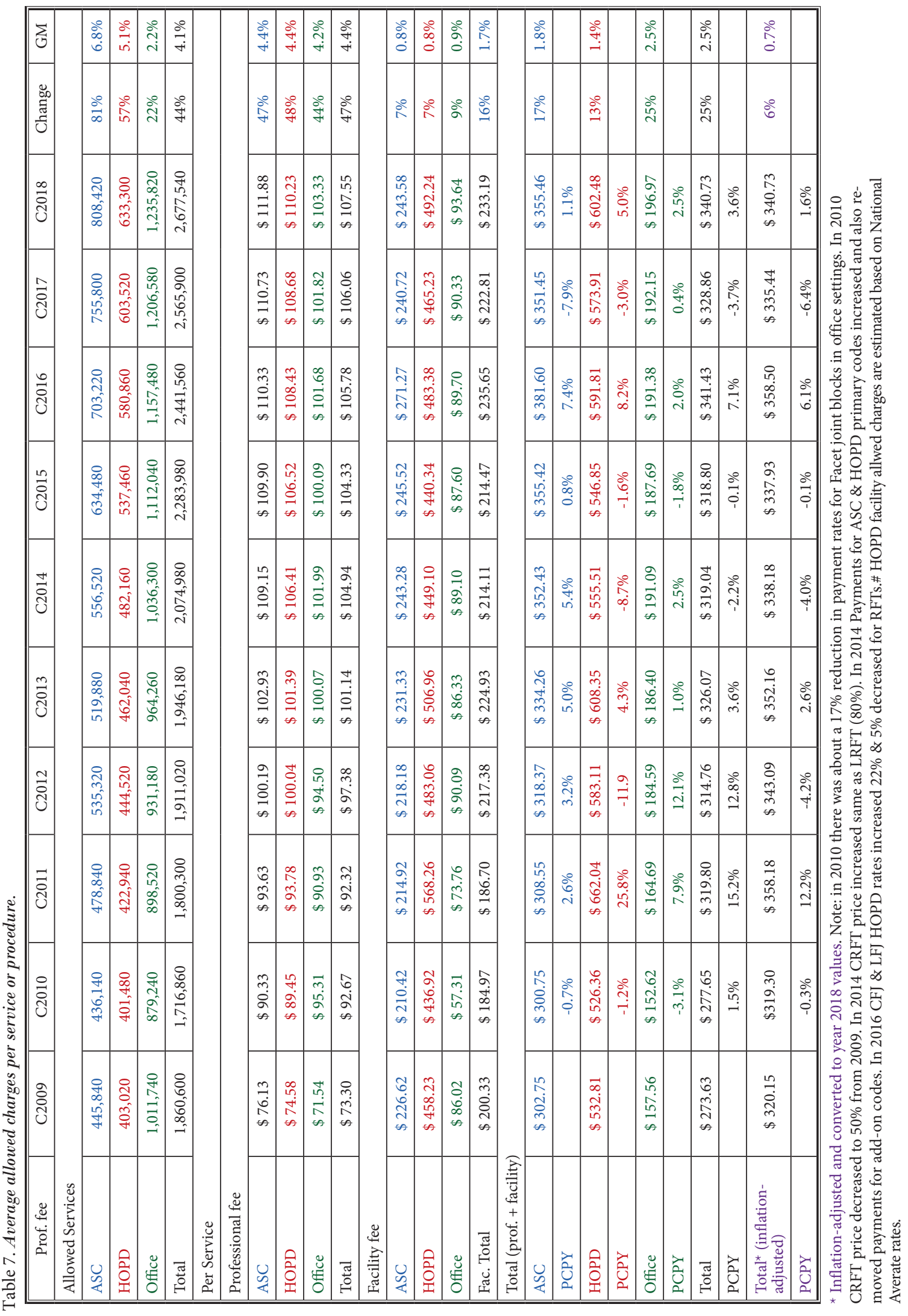


numbers in states covered in the past by First Coast Services state, Florida, followed by Novitas with the least in NGS Medicare Administrative Contractor (MAC). Appendix Tables 3 and 4 show the utilization patterns of lumbar/sacral facet joint nerve blocks compared to lumbar/sacral facet neurolysis with the reversal of usage patterns and significant growth patterns for facet neurolysis compared to facet joint injections. Appendix Tables 5 and 6 show the utilization patterns separately for cervical/thoracic facet joint nerve blocks compared to cervical/thoracic facet neurolysis. As shown earlier, the differences are significant with the growth of $8.7 \%$ for facet neurolysis and a $5.6 \%$ annual increase for facet joint nerve blocks. For cervical/thoracic, it is a significant growth of $112 \%$. Lumbar facet joint nerve blocks increased only $0.1 \%$ annually with a total increase of $0.6 \%$ from 2009 to 2018 . However, there was a significant increase in some states and consequently MACs. The largest declines were seen in Florida for First Coast Services with an annual decline of $2 \%$ and an overall decline of $16.4 \%$. However, their baseline utilization was the highest of all states with 1,453 per 100,000 population in 2009. This was followed by Noridian with an overall decline of $8.5 \%$ and WPS with a decline of $5.9 \%$ andwith the states covered by Cahaba in 2016 with $6.2 \%$. Increases were observed at a high rate of $23.5 \%$ with an annual increase of $2.4 \%$ in Palmetto MAC. In reference to lumbar facet neurolysis rates of utilization, all MACs showed increases with an overall increase of $97.7 \%$ across the United States and a $7.9 \%$ increase per year. Among the MACs, Novitas showed the highest increases with $123.2 \%$ or $9.3 \%$ annually per 100,000 FFS Medicare population. All MACs showed significant increases in utilization of radiofrequency neurotomy procedures. Perhaps, surprisingly, Palmetto MAC showed similar increases as other states.

The same pattern was observed with lumbar facet joint nerve blocks.

\section{Discussion}

The present analysis of facet joint intervention expenditure trends in the FFS Medicare population from 2009 to 2018 shows an overall inflation-adjusted to 2018 expenditures of $53 \%$ with a $4.9 \%$ annual rate, in contrast to unadjusted increases of $79 \%$ or $6.2 \%$ annually. The growth of the US Medicare population was $30.1 \%$ or $3 \%$ annually, with an increase of total patients undergoing facet joint interventions of $65.1 \%$ or $5.7 \%$ annually, visits increasing by $71.5 \%$ or $6.2 \%$ annually, and, finally, services increased by $43.9 \%$ or $4.1 \%$ annually. In contrast, the population-adjusted increase in rate of patients per 100,000 Medicare beneficiaries was $26.8 \%$ or $2.7 \%$ annually. Visits were $31.8 \%$ or $3.1 \%$ annually. Number of episodes were $21.6 \%$ or $2.2 \%$ annually, and, finally, services (procedures) were $10.6 \%$ or $1.1 \%$ annually. Further analysis also shows that the increase of unadjusted expenditures for cervical facet joint nerve blocks was $37 \%$, and for lumbar facet joint nerve blocks 35\%, whereas for cervical radiofrequency, increases were $185 \%$, and for lumbar radiofrequency neurolysis, increases were $169 \%$ with significant discrepancy of utilization and patterns and trends in the expenditures. This analysis also demonstrated unadjusted costs of $\$ 1.3$ million in 2009 and $\$ 1.53$ million in 2018, with an $18 \%$ increase per 100,000 FFS Medicare beneficiaries. Similarly, inflation-adjusted per patient expenditures also showed a decline from $\$ 1,925$ in 2009 to $\$ 1,785$, with a net decline of $7 \%$ from 2009 to 2018 and $0.8 \%$ per year. The present analysis also showed in 2018 , in $23.9 \%$ of the patients, episodes were staged, whereas, episodes beyond approval were performed in $6.9 \%$ of the patients with $68.9 \%$ of the patients receiving appropriate number of visits in lumbar spine. In contrast, in the cervical spine, these episodes were less with $19.6 \%$ of the patients undergoing staging, whereas, $5.1 \%$ of the patients receiving more than 2 episodes of radiofrequency neurotomy in 2018.

Overall, the present analysis showed inflationadjusted costs of $53 \%$ and $4.9 \%$ per year compared to $79 \%$ and $6.7 \%$ per procedure costs of $6 \%$ and $0.7 \%$ with $7 \%$ decline and $0.8 \%$ per year per patient, and $4 \%$ and $0.5 \%$, and decline of $11 \%$ and $1.3 \%$ per year per visit.

Overall increases in utilization of facet joint interventions is higher than the increase in population and expenditures adjusted for inflation. Increases in various categories include radiofrequency neurolysis, the unadjusted costs of which increased $185 \%$ for cervical neurolysis procedures and $169 \%$ for lumbar neurolysis procedures. In contrast, increases were 37\% for cervical facet joint injections and 35\% for lumbar facet joint injections even after inflation-adjusted overall increases were $53 \%$ with an annual increase of $4.9 \%$. Medicare beneficiary growth may attribute to an unknown percentage in the range of less than $10 \%$ in utilization patterns and expenditures. However, the overall costs per patient and visits have declined even though proce- 
dural costs have increased slightly above inflation. The inference is that the costs are due to increasing number of patients' visits, episodes of treatment, most importantly, enormous increases reflected in increase of the radiofrequency neurolysis procedures. This is illustrated by the data which showed diminution of ratio of facet joint nerve blocks to radiofrequency thermoneurolysis from 4.9 for cervical facet joint interventions and 3.8 for lumbar facet joint interventions to 2.4 for cervical and $\mathbf{1 . 9}$ for lumbar facet interventions.

Our data is in agreement with the data published in the Medicare population by Manchikanti et al from 2000 to 2008 (2). However, since 2009, trends in utilization analyzed expenditures have declined. These results reinforce our previous assessments of growth of facet joint interventions (3) which showed substantial increases from 2000 to 2009 and flattening of the growth and some declines from 2009 to 2018 as shown in Fig. 3. The trends in the utilization patterns, as well as the costs also follow similar patterns.

Our investigation is also in agreement with the assessment by Starr et al (5). They assessed trends in utilization and cost patterns of lumbar facet joint injections with radiofrequency neurolysis procedures. From 2007 to 2016, lumbar radiofrequency ablation sessions performed per 100,000 enrollees per year increased from 49 to 113 , a $130.6 \%$ overall increase,
$9.7 \%$ annually in commercially insured patients. In contrast they showed that lumbar facet joint injections increased from 201 to 251 sessions per 100,000 enrollees at a $24.9 \%$ overall increase, with an annual increase of $2.5 \%$. The costs also followed the same pattern. The unadjusted cost per 100,000 enrollees increased from $\$ 94,570$ in 2007 to $\$ 206,680$ in 2006, a $12.2 \%$ annual increase for lumbar radiofrequency ablation. Further, they also showed that unadjusted costs of lumbar facet joint injections were more with an annual increase of $4.9 \%$ and cost $\$ 257,280$ in 2007 increasing to $\$ 396,580$ in 2016 per 100,000 enrollees. However, if these costs are adjusted to inflation and 2016 -dollar value, with inflation of $25 \%$, changes will be lower.

Medicare is concerned with utilization patterns and increasing expenditures of facet joint interventions. Consequently, CMS continues to update LCDs, perform enhanced audits, and investigations (6-8,5661). At present, there is a push to reduce utilization and expenditures by not only reducing fraud and abuse, but also by enforcing appropriate indications and medical necessity criteria. During the enactment of the Affordable Care Act (ACA) (72-76) they have enacted LCDs which were essentially accepted verbatim by all carriers except for CGS, which kept the frequency at the same levels as prior years $(6-8,55-60)$. Since then,

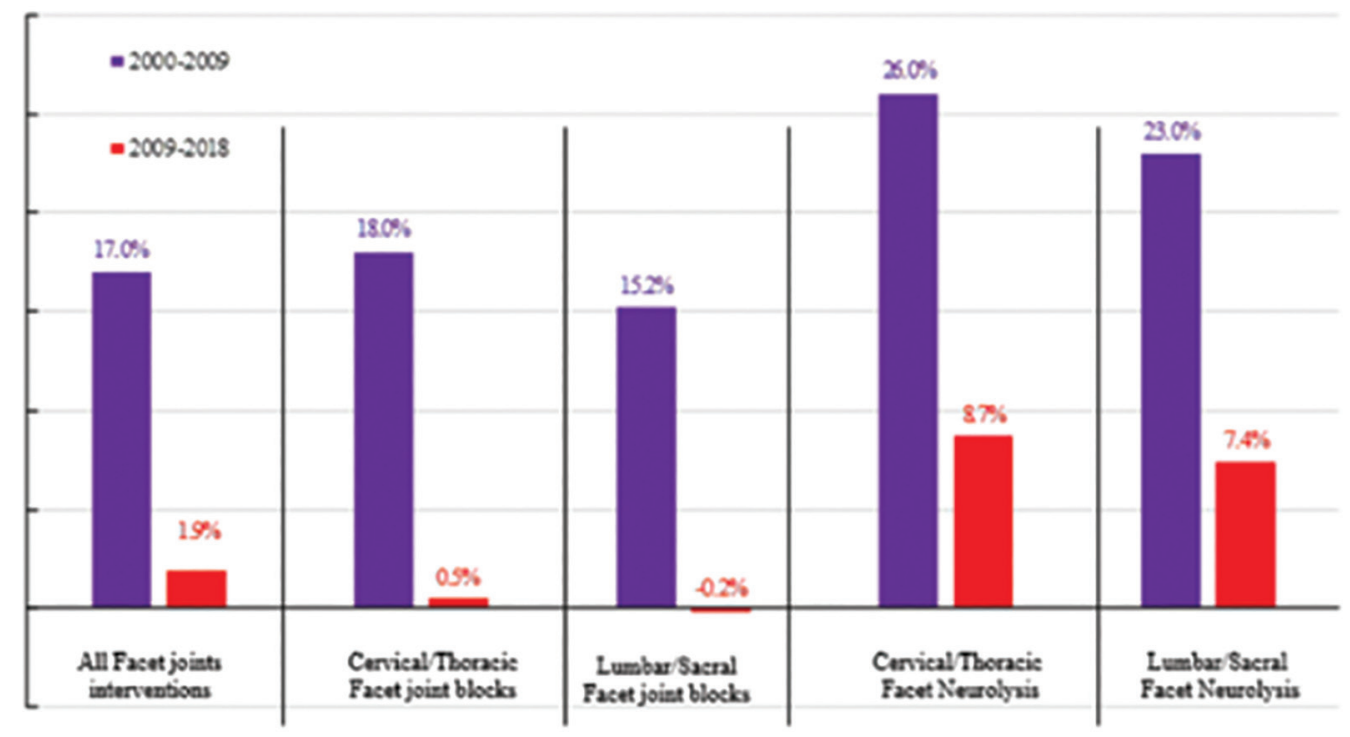

Fig. 3. Comparative utilization patterns based on an annual rate from 2000-2009 and 2009-2018. 
Palmetto (59) also has reduced the criterion standard for positive blocks prior to therapeutic interventions from $80 \%$ to $50 \%$. This change appears to have increased utilization patterns in Palmetto MAC for facet joint injections of $23.5 \%$ at annual increase of $2.4 \%$, while the majority of the other MACs either slightly increased or reduced utilization. There was no significant change in utilization of radiofrequency neurolysis procedures. These factors may have increased the utilization of radiofrequency neurotomy, which in itself is a more expensive procedure, while we started observing continued flattening and decline in utilization patterns for facet joint injections. In fact, transforaminal epidural injections have shown a significant increase in utilization patterns compared to caudal and interlaminar epidural injections as shown in Fig. 4. As the causes described above, e.g., overutilization, abuse, and fraud may be contributing to some beyond expected increases related to a grown Medicare population. Further, the arguments continue in reference to indications and medical necessity (5-8,40-61). However, the disagreements and criticisms are not limited to only the positive evidence, but also to negative evidence. There was a barrage of criticism (65-68) for a study published by Juch et al (64) regarding inappropriate performance of multiple elements of the trial, including technical aspects, selection and reporting criteria (65-68). Meanwhile, the criticism also has been advanced against multiple descriptions in the past of the appropriateness of criteria of controlled comparative local anesthetic blocks, $50 \%, 80 \%$ or $100 \%$ relief criterion, the duration of relief with diagnostic blocks, appropriateness of therapeutic facet joint neve blocks, and multiple procedural aspects for radiofrequency neurotomy (40-47,77-92).

\section{Conclusion}

This analysis of the FFS Medicare population from 2009 to 2018 demonstrated increasing costs for facet joint interventions, specifically radiofrequency neurolysis procedures, the ratios of which compared to facet joint injections, have changed significantly, or rather reversed. Even after adjusting for inflation, there is a significant increase in the expenditures of facet joint interventions with an overall $53 \%$ increase, and annual increase of $4.9 \%$. This study also showed $23.9 \%$ of the patients received staged episodes, whereas, $6.9 \%$ of the patients received more than 2 episodes of treatments beyond the number allowed by LCDs. In lumbar spine in
2018 with $19.6 \%$ staged and $5.1 \%$ receiving more than allowed in cervical spine.

\section{Acknowledgments}

The authors also wish to thank Bert Fellows, MA, Director Emeritus of Psychological Services, Pain Management Centers of America, for manuscript review, and Tonie M. Hatton and Diane E. Neihoff, transcriptionists, for their assistance in preparation of this manuscript. We would like to thank the editorial board of Pain Physician for review and criticism in improving the manuscript.

\section{Author Contributions}

The study was designed by LM, AS, and JAH.

Data collection and analysis was performed by VP.

All authors contributed to preparation to the manuscript, reviewed, and approved the content with final version.

\section{Author Affiliations}

Dr. Manchikanti is Co-Director, Pain Management Centers of America, Clinical Professor, Anesthesiology and Perioperative Medicine, University of Louisville, Louisville, KY, and Professor of AnesthesiologyResearch, Department of Anesthesiology, School of Medicine, LSU Health Sciences Center, New Orleans, LA. Vidyasagar Pampati is a Statistician, Pain Management Centers of America. Dr. Soin is Medical Director, Ohio Pain Clinic and Clinical Assistant Professor of Surgery at Wright State University, Dayton, $\mathrm{OH}$ Assistant Professor, Ohio University College of Medicine, Athens, $\mathrm{OH}$, and, Clinical Professor of Pain Management and Anesthesiology, University of Pikeville, Pikeville, KY. Dr. Vanaparthy is an Experiential Volunteer, Oregon Health and Science University, Portland, OR. Dr. Sanapati is CoDirector, Pain Management Centers of America Evansville, IN. Dr. Kaye is Vice-Chancellor of Academic Affairs, Chief Academic Officer, and Provost, Tenured Professor of Anesthesiology and Pharmacology, Toxicology, and Neurosciences, LSU School of Medicine, Shreveport, LA, Professor of Anesthesiology and Pharmacology, LSU School of Medicine, New Orleans, LA, and Professor of Anesthesiology and Pharmacology, Tulane School of Medicine, New Orleans, LA. Dr. Hirsch is Vice Chair and Service Line Chief of Neurointerventional Radiology, Chief of Neurointerventional Spine, Massachusetts General Hospital and Harvard Medical School, Boston, MA. 
Appendices

Appendix Table 1.

Appendix Table 2

Appendix Table 3

Appendix Table 4

Appendix Table 5

Appendix Table 6 


\section{References}

1. Manchikanti L, Sanapati MR, Pampati V, Boswell MV, Kaye AD, Hirsch JA. Update on reversal and decline of growth of utilization of interventional techniques in managing chronic pain in the Medicare population from 2000 to 2018. Pain Physician 2019; 22:521 536.

2. Manchikanti L, Pampati V, Falco FJ, Hirsch JA. Growth of spinal interventional pain management techniques: analysis of utilization trends and Medicare expenditures 2000 to 2008. Spine (Phila Pa 1976) 2013; 38:1570168.

3. Manchikanti L, Sanapati M, Pampati V, et al. Update of utilization patterns of facet joint interventions in managing spinal pain from 2000 to 2018 in the US fee-for-service Medicare population. Pain Physician 2020; 23:E133-E149.

4. Cohen SP, Bhaskar A, Bhatia A, et al. Consensus practice guidelines on interventions for lumbar facet joint pain from a multispecialty, international working group._Reg Anesth Pain Med 2020 [Epub ahead of print].

5. Starr JB, Gold L, McCormick Z, Suri $P$, Friedly J. Trends in lumbar radiofrequency ablation utilization from 2007 to 2016. Spine J 2019; 19:1019-1028.

6. Noridian Healthcare Solutions, LLC. LCD for facet joint injections, medial branch blocks, and facet joint radiofrequency neurotomy ( $L$ 34993). Revision Effective Date: 12/1/2019.

7. Manchikanti L, Falco FJE, Benyamin RM, Gharibo CG, Candido KD, Hirsch JA. Epidural steroid injections safety recommendations by the MultiSociety Pain Workgroup (MPW): More regulations without evidence or clarification. Pain Physician 2014; 17: $E_{575}-E_{5} 88$.

8. Manchikanti L, Helm II S, Singh V, Hirsch JA. Accountable interventional pain management: A collaboration among practitioners, patients, payers, and government. Pain Physician 2013; 16:E635-E670.

9. Dieleman JL, Cao J, Chapin A, et al. US health care spending by payer and health condition, 1996-2016. JAMA 2020; 323:863-884.

10. Dieleman JL, Baral R, Birger M, et al. US spending on personal health care and public health, 1996-2013. JAMA 2016; 316:2627-2646.
11. Centers for Medicare \& Medicaid Services NHE Fact Sheet.

https://www.cms.gov/research-statisticsdata-and-systems/statistics-trendsandreports/nationalhealthexpenddata/nhefact-sheet.html

12. Sisko AM, Truffer CJ, Keehan SP, Poisal JA, Clemens MK, Madison AJ. National health spending projections: The estimated impact of reform through 2019. Health Aff (Millwood) 2010; 29:1933-1941.

13. Cubanski J, Neuman T, and Freed M. The Facts on Medicare Spending and Financing. August 20, 2019.

http://files.kff.org/attachment/IssueBrief-Facts-on-Medicaid-Spending-andFinancing

14. Phelps A, Thomas S, Cruse CB. Setting the stage for Medicare for all and the health coverage debate. Deloitte Insights. January 29, 2020.

https://www2.deloitte.com/content/ $\mathrm{dam} /$ insights/us/articles/6400 Medicare-for-all/DI_Medicare\%20 for\%2oall.pdf

15. Nicola M, Alsafi Z, Sohrabi C, et al. The socio-economic implications of the coronavirus and COVID-19 pandemic: A review. Int J Surg 2020: 20:30316-30320.

16. Kabir M, Afzal MS, Khan A, Ahmed H. COVID-19 pandemic and economic cost; Impact on forcibly displaced people. Travel Med Infect Dis 2020 Apr 6. [Epub ahead of print]

17. US Burden of Disease Collaborators, Mokdad AH, Ballestros K, Echko M, et al. The State of US Health, 1990-2016: Burden of Diseases, Injuries, and Risk Factors Among US States. JAMA 2018; 319:1444-1472.

18. Rajaee SS, Bae HW, Kanim LE, Delamarter RB. Spinal fusion in the United States: Analysis of trends from 1998 to 2008. Spine (Phila Pa 1976) 2012; 37:67-76.

19. Deyo RA. Fusion surgery for lumbar degenerative disc disease: Still more questions than answers. Spine J 2015; 15:272-274.

20. Pannell WC, Savin DD, Scott TP, Wang JC, Daubs MD. Trends in the surgical treatment of lumbar spine disease in the United States. Spine J 2015; 15:1719-1727.

21. Jonan $A B$, Kaye $A D$, Urman RD. Buprenorphine formulations:
Clinical best practice strategies recommendations for perioperative management of patients undergoing surgical or interventional pain procedures. Pain Physician 2018; 21:E1-E12.

22. Harirforoosh S, Asghar W, Jamali F. Adverse effects of nonsteroidal antiinflammatory drugs: An update of gastrointestinal, cardiovascular and renal complications. J Pharm Pharm Sci 2013; 16:821-847.

23. U.S. Food and Drug Administration. FDA warms about serious breathing problems with seizure and nerve pain medications gabapentin (Neurontin, Gralise, Horizant) and pregabalin (Lyrica, Lyrica CR) when used with CNS depressants or in patients with lung problems. December 19, 2019.

24. Moore A, Wiffen P, Kalso E. Antiepileptic drugs for neuropathic pain and fibromyalgia. JAMA 2014; 312:182-183.

25. Leavitt SB. NSAID dangers may limit pain-relief options. Pain-Topics News/ Research UPDATES, March 14, 2010.

http://updates.pain-topics.org/2010/03/ nsaid-dangers-may-limit-pain-relief. html

26. Manchikanti L, Kaye AM, Knezevic NN, et al. Responsible, safe, and effective prescription of opioids for chronic non-cancer pain: American Society of Interventional Pain Physicians (ASIPP) guidelines. Pain Physician 2017; 20: $\mathrm{S}_{3}-\mathrm{S}_{92}$.

27. Hopkins RE, Bui T, Magliano D, Arnold C, Dooley M. Prescriber education interventions to optimize opioid prescribing in acute care: A systematic review. Pain Physician 2019; 22:E551-E562.

28. Helm II S, Racz G. Hyaluronidase in neuroplasty: A review. Pain Physician 2019; 22:555-560.

29. Manchikanti L, Soin A, Mann DP, et al. Utilization patterns of facet joint interventions in managing spinal pain: A retrospective cohort study in the US fee-for-service Medicare population. Curr Pain Headache Rep 2019; 23:73.

30. Manchikanti L, Soin A, Mann DP, Bakshi S, Pampati V, Hirsch JA. Comparative analysis of utilization of epidural procedures in managing chronic pain in the Medicare population: Pre and post Affordable Care Act. Spine (Phila Pa 1976) 2019; 44:220-232. 
31. Manchikanti L, Sanapati MR, Soin A, et al. An updated analysis of utilization of epidural procedures in managing chronic pain in the Medicare population from 2000 to 2018. Pain Physician 2020; 12:111-126.

32. Manchikanti MV, Manchikanti L, Kaye AD, Pampati V, Hirsch JA. Usage patterns of sacroiliac joint injections - A comparative evaluation of pre and post Affordable Care Act in Medicare population. IPM Reports 2018; 2:157-166.

33. Manchikanti L, Sanapati J, Pampati V, Kaye AD, Hirsch JA. Utilization of vertebral augmentation procedures in the United States: A comparative analysis in Medicare fee-for-service population pre- and post-2009 trials. Curr Pain Headache Rep 2019; in press.

34. Farber SH, Han JL, Petraglia III FW, et al. Increasing rates of imaging in failed back surgery syndrome patients: Implications for spinal cord stimulation. Pain Physician 2017; 20:E969-E977.

35. Farber $\mathrm{SH}$, Han JL, Elsamadicy AA, et al. Long-term cost utility of spinal cord stimulation in patients with failed back surgery syndrome. Pain Physician 2017; 20:E797-E805.

36. Sanger $N$, Bhatt $M$, Singhal $N$, et al. Adverse outcomes associated with prescription opioids for acute low back pain: A systematic review and metaanalysis. Pain Physician 2019; 22:119-138.

37. Moride $Y$, Lemieux-Uresandi $D$ Castillon $G$, et al. A systematic review of interventions and programs targeting appropriate prescribing of opioids. Pain Physician 2019; 22:229-240.

38. Kertesz SG, Gordon AJ. A crisis of opioids and the limits of prescription control: United States. Addiction 2019; 114:169-180.

39. Gever J. HHS: Don't withdraw opioids suddenly-Department issues guideline on tapering and discontinuation. MedPage Today, October 10, 2019.

40. Boswell MV, Manchikanti L, Kaye AD, et al. A best-evidence systematic appraisal of the diagnostic accuracy and utility of facet (zygapophysial) joint injections in chronic spinal pain. Pain Physician 2015; 18: E497-E533.

41. Manchikanti L, Kaye AD, Boswell MV, et al. A systematic review and best evidence synthesis of the effectiveness of therapeutic facet joint interventions in managing chronic spinal pain. Pain Physician 2015; 18: $E_{535}-E_{5} 82$.

42. Manchikanti L, Pampati V, Kaye AD,
Hirsch JA. Therapeutic lumbar facet joint nerve blocks in the treatment of chronic low back pain: Cost utility analysis based on a randomized controlled trial. Korean J Pain 2018; 31:27-38.

43. Manchikanti L, Pampati V, Kaye AD, Hirsch JA. Cost utility analysis of cervical therapeutic medial branch blocks in managing chronic neck pain. Int J Med Sci 2017; 14:1307-1316.

44. Manchikanti L, Abdi S, Atluri S, et al. An update of comprehensive evidencebased guidelines for interventional techniques of chronic spinal pain: Part II: Guidance and recommendations. Pain Physician 2013; 16:S49-S283.

45. Manchikanti L, Hirsch JA, Kaye AD, Boswell MV. Cervical zygapophysial (facet) joint pain: Effectiveness of interventional management strategies. Postgrad Med 2016; 128:54-68.

46. Manchikanti L, Hirsch JA, Falco FJE, Boswell MF. Management of lumbar zygapophysial (facet) joint pain. World J Orthop 2016; 7:315-337.

47. Song K, Li Z, Shuang F, et al. Comparison of the effectiveness of radiofrequency neurotomy and endoscopic neurotomy of lumbar medial branch for facetogenic chronic low back pain: A randomized controlled trial. World Neurosurg 2019; 126:e109-e115

48. Lee JH, Shin KS, Park SJ, et al. Comparison of clinical efficacy between transforaminal and interlaminar epidural injections in lumbosacral disc herniation: A systematic review and meta-analysis. Pain Physician 2018; 21:433-448.

49. Lee JH, Kim DH, Kim DH, et al. Comparison of clinical efficacy of epidural injection with or without steroid in lumbosacral disc herniation: A systematic review and meta-analysis. Pain Physician 2018; 21:449-468.

50. Manchikanti L, Pampati V, Benyamin RM, Hirsch JA. Cost utility analysis of lumbar interlaminar epidural injections in the treatment of lumbar disc herniation, central spinal stenosis, and axial or discogenic low back pain. Pain Physician 2017; 20:219-228.

51. Manchikanti L, Helm S 2nd, Pampati V, Racz GB. Cost utility analysis of percutaneous adhesiolysis in managing pain of post-lumbar surgery syndrome and lumbar central spinal stenosis. Pain Pract 2015; 15:414-422.

52. Manchikanti L, Pampati V, Parr III A, et al. Cervical interlaminar epidural injections in the treatment of cervical disc herniation, post surgery syndrome, or discogenic pain: Cost utility analysis from randomized trials. Pain Physician 2019; 22:421-431.

53. Sanapati J, Manchikanti L, Atluri S, et al. Do regenerative medicine therapies provide long-term relief in chronic low back pain: A systematic review and metaanalysis. Pain Physician 2018; 21:515-540.

54. Chakravarthy K, Manchikanti L, Kaye AD, Christo PJ. Reframing the role of neuromodulation therapy in the chronic pain treatment paradigm. Pain Physician 2018; 21:507-513.

55. National Government Services, Inc. LCD for facet joint injections, medial branch blocks, and facet joint radiofrequency neurotomy (L35936). Revision Effective Date: 12/05/2019.

56. Novitas Solutions, Inc. LCD for facet joint interventions for pain management (L34892). Revision Effective Date: 11/21/2019.

57. First Coast Services Options, Inc. Local Coverage Determination (LCD): Paravertebral Facet Joint Blocks (L33930). Revision Effective Date 01/08/2019.

58. Wisconsin Physician Service Insurance Corporation. Facet Joint Injections, Medial Branch Blocks, and Facet Joint Radiofrequency Neurotomy (L35996). Revision Effective Date 11/01/2019.

59. Palmetto GBA. Local Coverage Determination (LCD): Facet Joint Injections, Medial Branch Blocks, and Facet Joint Radiofrequency Neurotomy (L36471). Revision Effective Date 10/10/2019.

60. CGS Administrators, LLC. Local Coverage Determination (LCD). Facet Joint Injections, Medial Branch Blocks, and Facet Joint Radiofrequency Neurotomy (L34832). Original Effective Date 10/01/2015; Revision Effective Date: $12 / 26 / 2019$.

61. US Department of Health and Human Services. Office of Inspector General (OIG). Medicare Payments for Facet Joint Injection Services (OEI-05-0700200). September 2008.

http://www.oig.hhs.gov/oei/reports/oei05-07-00200.pdf

62. Tosteson AN, Skinner JS, Tosteson $T D$, et al. The cost effectiveness of surgical versus nonoperative treatment for lumbar disc herniation over two years: Evidence from the Spine Patient Outcomes Research Trial (SPORT). Spine 
(Phila Pa 1976) 2008; 33:2108-2115

63. Tosteson AN, Lurie JD, Tosteson TD, et al; SPORT Investigators. Surgical treatment of spinal stenosis with and without degenerative spondylolisthesis: Cost-effectiveness after 2 years. Ann Intern Med 2008; 149:845-853.

64. Juch JNS, Maas ET, Ostelo RWJG, et al. Effect of radiofrequency denervation on pain intensity among patients with chronic low back pain: The Mint randomized clinical trials. JAMA 2017; 318:68-81.

65. Provenzano DA, Buvanendran A, De Leon-Casasola O, Narouze S, Cohen SP. Interpreting the MINT randomized clinical trials: let us stick to the facts. Reg Anesth Pain Med 2020; 45:84-86.

66. van Kuijk SMJ, Van Zundert J, Hans $\mathrm{G}$, et al. Flawed study design and incorrect presentation of data negatively impact potentially useful interventional treatments for patients with low back pain: A critical review of JAMA's MinT study. Pain Pract 2018; 18:292-295.

67. Rimmalapudi V, Buchalter J, Calodney A. radiofrequency denervation for chronic low back pain. JAMA 2017; 318:2255-2256.

68. Kao MC, Leong MS, Mackey S. Radiofrequency denervation for chronic low back pain. JAMA 2017; 318:2256.

69. Manchikanti L, Atluri S, Albers SL, Abd-Elsayed A, et al. Comprehensive evidence-based guidelines for facet joint interventions in the management of chronic spinal pain: American Society of Interventional Pain Physicians (ASIPP) guidelines. Pain Physician 2020; 23:S1-S132.

70. Vandenbroucke JP, von Elm E, Altman DG, et al; STROBE Initiative. Strengthening the Reporting of Observational Studies in Epidemiology (STROBE): Explanation and elaboration. Epidemiology 2007; 18:805-835.

71. Centers for Medicare and Medicaid Services. SShttps://www.cms.gov/

72. Manchikanti L, Helm S 2nd, Benyamin RM, et al. A critical analysis of Obamacare: affordable care or insurance for many and coverage for few? Pain Physician 2017; 20:111-138.

73. Cannon MF. Is Obamacare harming quality? (Part 1). Health affairs blog, January 4, 2018. https://www. healthaffairs.org do/10.1377/hblog20180103.261091/full/

74. Blumenthal D, Collins SR, Fowler EJ. The Affordable Care Act at 10 Years - Its coverage and access provisions. $N$ Engl J Med 2020; 382:963-969.

75. Manchikanti L, Singh V, Benyamin RM, Kaye AD, Pampati V, Hirsch JA. Reframing Medicare physician payment policy for 2019: A look at proposed policy. Pain Physician 2018; 21:415-432.

76. Nguyen TH, Milburn JM, Duszak R, Savoie J, HorDy M, Hirsch JA. Medicare for All: Considerations for Neuroradiologists. AJNR Am J Neuroradiol 2020 [Epub ahead of print].

77. Engel A, MacVicar J, Bogduk N. A philosophical foundation for diagnostic blocks, with criteria for their validation. Pain Med 2014; 15:998-1006.

78. Bogduk N. On diagnostic blocks for lumbar zygapophysial joint pain._F10oo Med Rep 2010;2:57.

79. Schwarzer AC, Aprill C, Derby R, Fortin J, Kine G, Bogduk N. Clinical features of patients with pain stemming from the lumbar zygapophyseal joints. Is the lumbar facet syndrome a clinical entity? Spine (Phila Pa 1976) 1994; 10:1132-1137.

80. Schwarzer AC, Wang S, Bogduk N, McNaught PJ, Laurent R. Prevalence and clinical features of lumbar zygapophysial joint pain: A study in an Australian population with chronic low back pain. Ann Rheum Dis 1995; 54:100-106.

81. Yin W, Bogduk N. The nature of neck pain in a private pain clinic in the United States. Pain Med 2008; 9:196-203.

82. Barnsley L, Lord SM, Wallis BJ, Bogduk $\mathrm{N}$. The prevalence of chronic cervical zygapophyseal joint pain after whiplash. Spine (Phila Pa 1976) 1995; 20:20-26.

83. Lord SM, Barnsley L, Wallis BJ, Bogduk N. Chronic cervical zygapophysial joint pain with whiplash: A placebocontrolled prevalence study. Spine (Phila Pa 1976) 1996; 21:1737-1744.

84. Cohen SP, Williams KA, Kurihara C, et al. Multicenter, randomized, comparative cost-effectiveness study comparing 0 , 1 , and 2 diagnostic medial branch (facet joint nerve) block treatment paradigms before lumbar facet radiofrequency denervation. Anesthesiology 2010; 113:395-405.
85. Cohen SP, Doshi TL, Constantinescu OC, et al. Effectiveness of lumbar facet joint blocks and predictive value before radiofrequency denervation: The Facet Treatment Study (FACTS), a randomized, controlled clinical trial. Anesthesiology 2018; 129:517-535.

86. Cohen SP, Moon JY, Brummett CM, White RL, Larkin TM. Medial Branch Blocks or Intra-articular injections as a prognostic tool before lumbar facet radiofrequency denervation: A multicenter, case-control study. Reg Anesth Pain Med 2015; 40:3760383.

87. Manchikanti L, Pampati S, Cash KA. Making sense of the accuracy of diagnostic lumbar facet joint nerve blocks: An assessment of the implications of $50 \%$ relief, $80 \%$ relief, single block, or controlled diagnostic blocks. Pain Physician 2010; 13:133-143.

88. Pampati S, Cash KA, Manchikanti L. Accuracy of diagnostic lumbar facet joint nerve blocks: A 2-year follow-up of 152 patients diagnosed with controlled diagnostic blocks. Pain Physician 2009; 12:855-866.

89. Manchikanti L, Pampati V, Fellows B, Bakhit CE. The diagnostic validity and therapeutic value of lumbar facet joint nerve blocks with or without adjuvant agents. Curr Rev Pain 2000; 4:337-344.

90. Manchikanti L, Singh V, Falco FJ, Cash KA, Pampati V. Lumbar facet joint nerve blocks in managing chronic facet joint pain: One-year follow-up of a randomized, double-blind controlled trial: Clinical Trial NCTo0355914. Pain Physician 2008, 11:121-132.

91. Manchikanti L, Singh V, Falco FJE, Cash KA, Pampati V, Fellows B. The role of thoracic medial branch blocks in managing chronic mid and upper back pain: A randomized, doubleblind, active-control trial with a 2-year follow-up. Anesthesiol Res Pract 2012; 2012:585806.

92. Manchikanti L, Singh V, Falco FJE, Cash KA, Fellows B. Comparative outcomes of a 2-year follow-up of cervical medial branch blocks in management of chronic neck pain: A randomized, double-blind controlled trial. Pain Physician 2010; 13:437-450. 



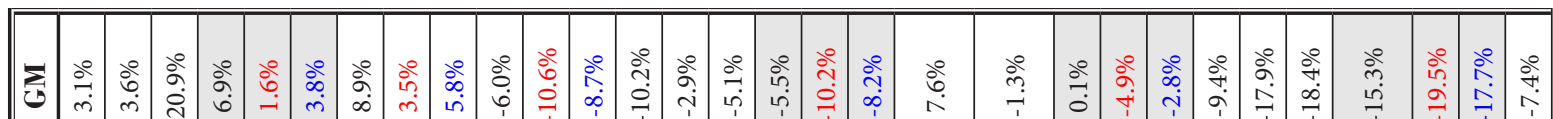

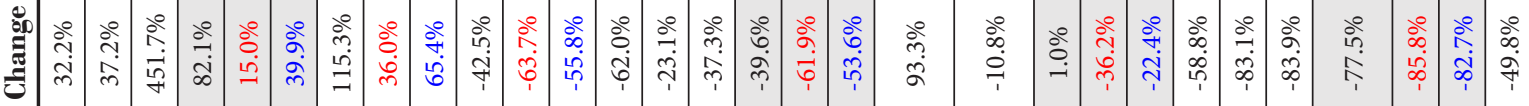

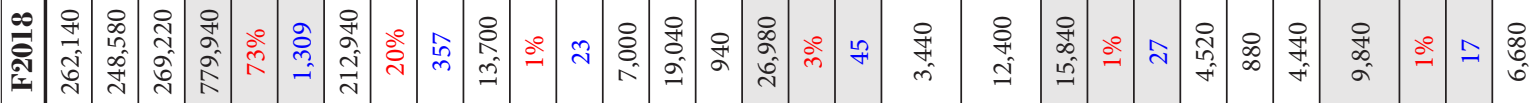

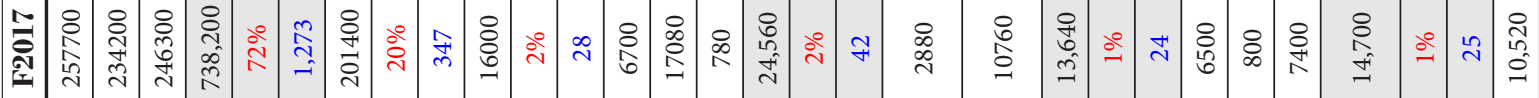

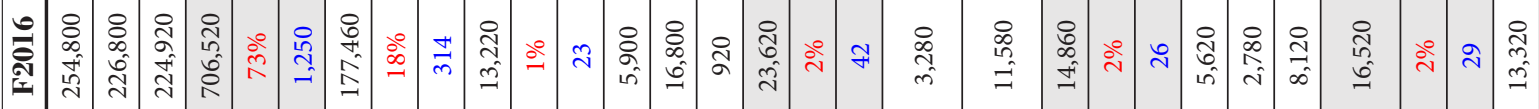

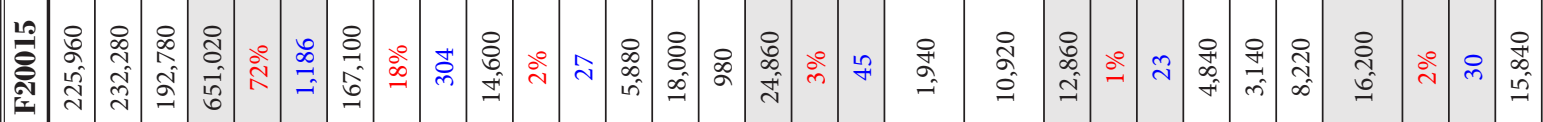

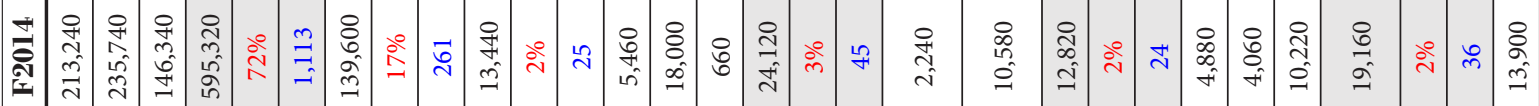

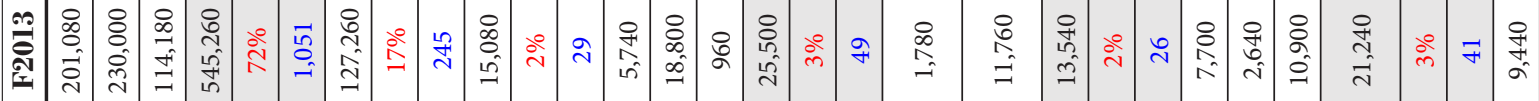

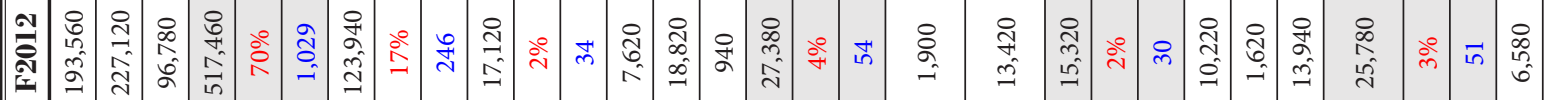

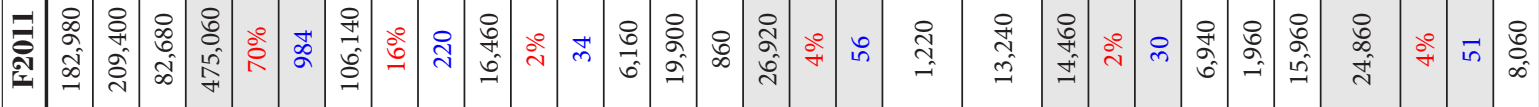

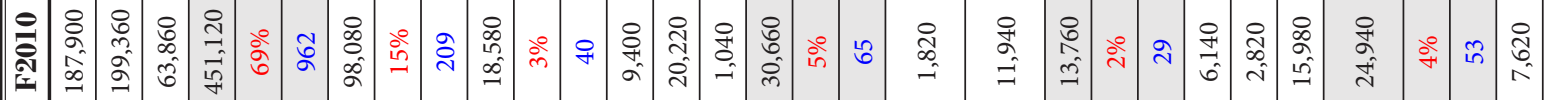

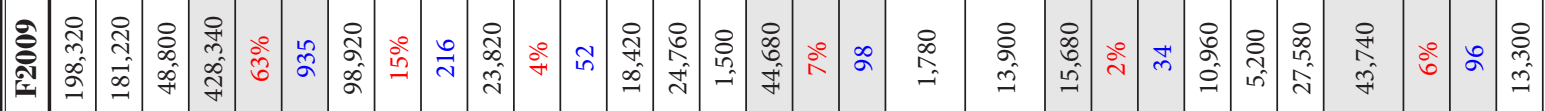

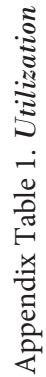

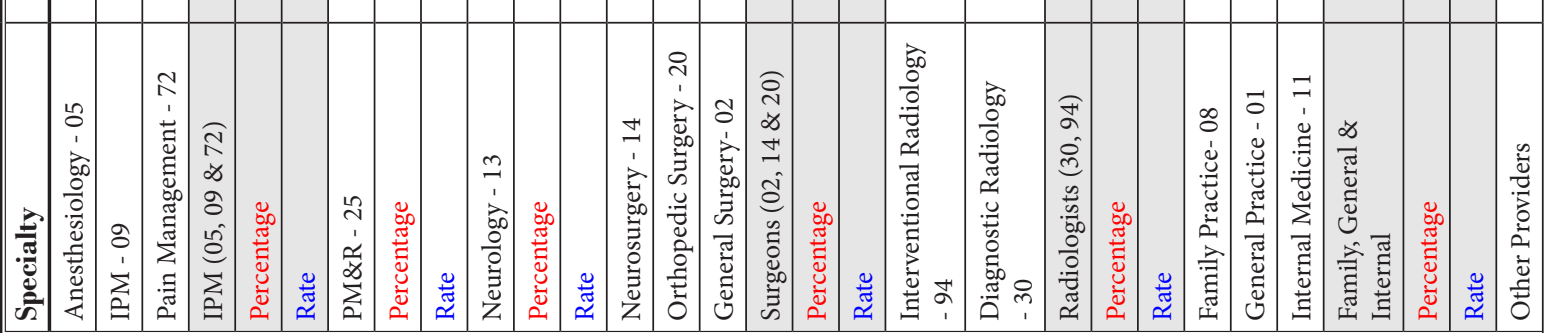




\begin{tabular}{|c|c|c|c|c|c|c|c|c|c|c|}
\hline$\sum_{0}^{0}$ & $\begin{array}{l}\stackrel{\circ}{0} \\
\stackrel{1}{7} \\
1\end{array}$ & 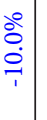 & ஸें & $\begin{array}{l}0 \\
\stackrel{0}{0} \\
\stackrel{+}{1} \\
\end{array}$ & 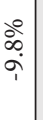 & $\begin{array}{c}\text { aें } \\
\text { कें }\end{array}$ & 总 & $\begin{array}{c}\stackrel{3}{\circ} \\
\stackrel{1}{\circ}\end{array}$ & ڤે̀ & ڤે̀ \\
\hline 密 & $\begin{array}{l}0 \\
\infty \\
\infty \\
0 \\
0 \\
1\end{array}$ & 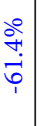 & $\begin{array}{c}\text { mे } \\
\dot{m}\end{array}$ & $\begin{array}{l}\text { oे } \\
\stackrel{0}{i} \\
i \\
1\end{array}$ & $\begin{array}{l}\text { خें } \\
\text { خे } \\
\text { فे }\end{array}$ & 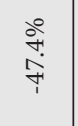 & \begin{tabular}{|l}
0 \\
0 \\
0 \\
0
\end{tabular} & $\begin{array}{l}30 \\
\text { के } \\
\text { ?े }\end{array}$ & 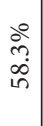 & $\begin{array}{l}\text { \&े } \\
\text { iे }\end{array}$ \\
\hline 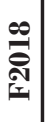 & $\stackrel{\circ}{\circ}$ & $\Rightarrow$ & 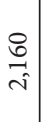 & ঃ & : & 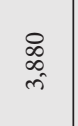 & 犬े & $\wedge$ & $\begin{array}{l}8 \\
0 \\
0 \\
0 \\
0 \\
-1 \\
-1\end{array}$ & 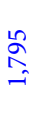 \\
\hline 䏠 & $\therefore$ & $\stackrel{\infty}{\sim}$ & $\begin{array}{l}\infty \\
\stackrel{\infty}{=}\end{array}$ & $\begin{array}{l}\infty \\
0 \\
0\end{array}$ & 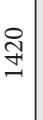 & $\begin{array}{l}\infty \\
\infty \\
\infty \\
\end{array}$ & $\begin{array}{c}\circ \\
\stackrel{\circ}{0} \\
0\end{array}$ & $\wedge$ & 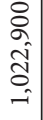 & 总 \\
\hline 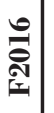 & $\stackrel{\circ}{\Rightarrow}$ & $\stackrel{\sim}{\sim}$ & 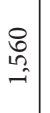 & $\stackrel{\Xi}{ }$ & : & $\frac{g}{m}$ & $\begin{array}{c}\text { के } \\
\text { ஸे }\end{array}$ & $\bullet$ & $\begin{array}{l}8 \\
0 \\
0 \\
0 \\
0 \\
0\end{array}$ & $\underset{-}{\mathbb{N}}$ \\
\hline 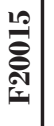 & $\stackrel{\circ}{\circ}$ & बे & \begin{tabular}{l}
$\stackrel{8}{0}$ \\
\hdashline \\
-1
\end{tabular} & $\begin{array}{l}\text { 이 } \\
\infty\end{array}$ & $\stackrel{\infty}{\triangleright}$ & $\begin{array}{l}\text { సิ } \\
\text { }\end{array}$ & $\begin{array}{c}\text { ¿ें } \\
\text { oे }\end{array}$ & in & $\begin{array}{l}8 \\
\vdots \\
+ \\
\hat{\alpha} \\
\alpha\end{array}$ & ఫे \\
\hline 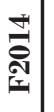 & $\stackrel{\circ}{\circ}$ & 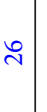 & 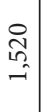 & $\stackrel{8}{\infty}$ & $\begin{array}{l}\stackrel{8}{0} \\
-1 \\
-1\end{array}$ & $\begin{array}{l}\stackrel{8}{0} \\
m^{2}\end{array}$ & $\begin{array}{c}\circ \\
\dot{H} \\
0\end{array}$ & 0 & 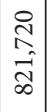 & 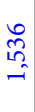 \\
\hline 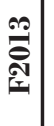 & $\stackrel{\circ}{\Rightarrow}$ & $\stackrel{\infty}{-1}$ & \begin{tabular}{c|c}
0 \\
$\substack{1 \\
-\\
-1}$
\end{tabular} & 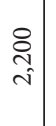 & 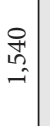 & $\underset{i n}{\stackrel{8}{15}}$ & $\mid \begin{array}{l}\circ \\
\grave{2} \\
0\end{array}$ & $\circ$ & 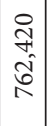 & ô \\
\hline 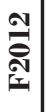 & 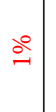 & $\cong$ & 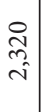 & $\begin{array}{l}8 \\
b \\
b \\
f^{\prime}\end{array}$ & $\begin{array}{c}0 \\
\mathbb{0} \\
i\end{array}$ & \begin{tabular}{l}
8 \\
\multirow{\infty}{\infty}{} \\
$\infty$
\end{tabular} & $\mid \begin{array}{c}\stackrel{\circ}{1} \\
\stackrel{-}{1}\end{array}$ & $\stackrel{\infty}{\sim}$ & 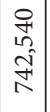 & 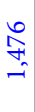 \\
\hline 司 & $\stackrel{\circ}{\circ}$ & $\approx$ & : & $\begin{array}{c}0 \\
0 \\
\infty \\
+ \\
+\end{array}$ & 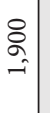 & $\underset{\sim}{\stackrel{7}{N}}$ & $\stackrel{\circ}{-}$ & $\stackrel{2}{2}$ & $\begin{array}{l}\infty \\
\infty \\
2 \\
\hat{\sigma} \\
\hat{\sigma}\end{array}$ & 产 \\
\hline 产 & $\stackrel{\circ}{=}$ & $\div$ & $\begin{array}{c}\stackrel{8}{=} \\
= \\
=\end{array}$ & 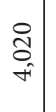 & $\begin{array}{l}\infty \\
\stackrel{D}{-1} \\
-1\end{array}$ & $\begin{array}{l}8 \\
\text { कू } \\
\text { bे }\end{array}$ & $\begin{array}{c}\stackrel{0}{-} \\
-1\end{array}$ & $\stackrel{2}{2}$ & $\begin{array}{l}0 \\
\hat{N} \\
\overrightarrow{\hat{D}} \\
0\end{array}$ & 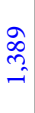 \\
\hline 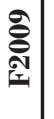 & $\stackrel{े}{\circ}$ & $\grave{\mathrm{i}}$ & - & $\begin{array}{l}0 \\
+1 \\
\infty \\
\overbrace{1}\end{array}$ & 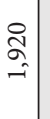 & 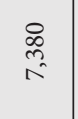 & $\stackrel{\circ}{\stackrel{-}{-}}$ & 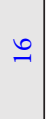 & $\begin{array}{l}0 \\
0 \\
\infty \\
\hat{N} \\
6\end{array}$ & 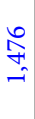 \\
\hline 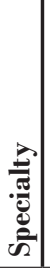 & 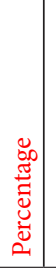 & $\stackrel{2}{\frac{0}{\pi}}$ & 忢 & 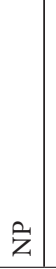 & $\overleftrightarrow{\Xi}$ & 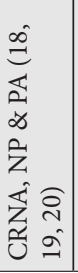 & 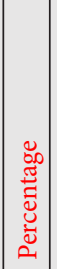 & 婄 & 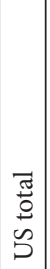 & ๕ँ \\
\hline
\end{tabular}


Trends of Expenditures of Facet Joint Interventions in Medicare Population

Appendix Table 2. Utilization of facet joint interventions rate (episodes) by Medicare carrier and state.

\begin{tabular}{|c|c|c|c|c|c|c|c|c|c|c|c|c|}
\hline State name & R2009 & R2010 & R2011 & R2012 & R2013 & R2014 & R2015 & R2016 & R2017 & R2018 & Change & GM \\
\hline \multicolumn{13}{|l|}{ Cahaba } \\
\hline Alabama & 1,438 & 1,824 & 1,766 & 1,989 & 1,774 & 1,808 & 1,752 & 2,025 & 1,755 & 1,794 & $24.8 \%$ & $2.5 \%$ \\
\hline Georgia & 1,883 & 1,923 & 1,952 & 2,176 & 2,110 & 2,160 & 2,361 & 2,393 & 2,368 & 2,466 & $31.0 \%$ & $3.0 \%$ \\
\hline Tennessee & 1,767 & 1,701 & 1,791 & 1,786 & 1,432 & 1,340 & 1,390 & 1,637 & 1,676 & 1,601 & $-9.4 \%$ & $-1.1 \%$ \\
\hline Cahaba Total & 1,723 & 1,822 & 1,848 & 1,996 & 1,793 & 1,793 & 1,878 & 2,046 & 1,982 & 2,010 & $16.7 \%$ & $1.7 \%$ \\
\hline PCPY & & $5.7 \%$ & $1.5 \%$ & $8.0 \%$ & $-10.2 \%$ & $0.0 \%$ & $4.7 \%$ & $9.0 \%$ & $-3.1 \%$ & $1.4 \%$ & & \\
\hline \multicolumn{13}{|l|}{ CGS } \\
\hline Kentucky & 2,018 & 1,819 & 1,998 & 2,191 & 2,213 & 2,039 & 2,431 & 2,427 & 2,663 & 2,685 & $33.1 \%$ & $3.2 \%$ \\
\hline Ohio & 1,237 & 1,310 & 1,487 & 1,605 & 1,682 & 1,626 & 1,767 & 1,852 & 1,720 & 1,741 & $40.7 \%$ & $3.9 \%$ \\
\hline CGS Total & 1,459 & 1,455 & 1,634 & 1,773 & 1,835 & 1,745 & 1,958 & 2,016 & 1,989 & 2,009 & $37.7 \%$ & $3.6 \%$ \\
\hline PCPY & & $-0.3 \%$ & $12.3 \%$ & $8.5 \%$ & $3.5 \%$ & $-4.9 \%$ & $12.2 \%$ & $3.0 \%$ & $-1.4 \%$ & $1.0 \%$ & & \\
\hline \multicolumn{13}{|l|}{ First Coast } \\
\hline Florida & 2,347 & 2,084 & 2,123 & 2,309 & 2,066 & 2,360 & 2,399 & 2,542 & 2,466 & 2,485 & $5.9 \%$ & $0.6 \%$ \\
\hline PCPY & & $-11.2 \%$ & $1.8 \%$ & $8.7 \%$ & $-10.5 \%$ & $14.2 \%$ & $1.7 \%$ & $5.9 \%$ & $-3.0 \%$ & $0.8 \%$ & & \\
\hline \multicolumn{13}{|l|}{ NGS } \\
\hline Connecticut & 1,064 & 1,078 & 971 & 1,050 & 1,106 & 1,143 & 1,232 & 1,164 & 1,350 & 1,405 & $32.0 \%$ & $3.1 \%$ \\
\hline Illinois & 1,396 & 1,018 & 1,207 & 1,390 & 1,311 & 1,346 & 1,417 & 1,411 & 1,548 & 1,528 & $9.4 \%$ & $1.0 \%$ \\
\hline Maine & 1,088 & 929 & 1,056 & 1,129 & 1,252 & 1,506 & 1,718 & 1,469 & 1,366 & 1,238 & $13.7 \%$ & $1.4 \%$ \\
\hline Massachusetts & 1,341 & 1,350 & 1,425 & 1,641 & 1,697 & 1,683 & 1,754 & 1,608 & 1,714 & 1,767 & $31.8 \%$ & $3.1 \%$ \\
\hline Minnesota & 678 & 545 & 679 & 854 & 795 & 819 & 814 & 765 & 761 & 809 & $19.3 \%$ & $2.0 \%$ \\
\hline New Hampshire & 1,233 & 1,693 & 1,957 & 1,962 & 1,368 & 1,615 & 1,606 & 1,923 & 1,808 & 1,806 & $46.5 \%$ & $4.3 \%$ \\
\hline New York & 849 & 789 & 805 & 754 & 966 & 1,028 & 1,170 & 1,216 & 1,157 & 1,174 & $38.3 \%$ & $3.7 \%$ \\
\hline Rhode Island & 1,387 & 1,202 & 1,293 & 955 & 1,188 & 1,043 & 1,042 & 1,122 & 832 & 1,146 & $-17.3 \%$ & $-2.1 \%$ \\
\hline Vermont & 1,612 & 1,327 & 1,256 & 1,295 & 1,501 & 1,827 & 1,717 & 1,918 & 1,939 & 1,588 & $-1.5 \%$ & $-0.2 \%$ \\
\hline Wisconsin & 1,121 & 1,091 & 1,192 & 1,360 & 1,333 & 1,294 & 1,363 & 1,470 & 1,410 & 1,416 & $26.3 \%$ & $2.6 \%$ \\
\hline NGS Total & 1,084 & 972 & 1,053 & 1,132 & 1,185 & 1,227 & 1,314 & 1,319 & 1,333 & 1,347 & $24.3 \%$ & $2.4 \%$ \\
\hline PCPY & & $-10.3 \%$ & $8.3 \%$ & $7.6 \%$ & $4.7 \%$ & $3.5 \%$ & $7.1 \%$ & $0.3 \%$ & $1.1 \%$ & $1.0 \%$ & & \\
\hline \multicolumn{13}{|l|}{ Noridian } \\
\hline Alaska & 1,180 & 974 & 697 & 491 & 988 & 1,383 & 1,760 & 1,812 & 1,633 & 1,933 & $63.8 \%$ & $5.6 \%$ \\
\hline Arizona & 1,723 & 2,103 & 1,924 & 2,153 & 2,247 & 2,157 & 2,427 & 2,429 & 2,840 & 3,056 & $77.4 \%$ & $6.6 \%$ \\
\hline California & 1,272 & 1,131 & 1,137 & 1,087 & 1,062 & 1,033 & 1,053 & 1,057 & 1,084 & 1,148 & $-9.7 \%$ & $-1.1 \%$ \\
\hline Idaho & 928 & 818 & 775 & 1,112 & 1,081 & 1,180 & 1,061 & 1,262 & 1,273 & 1,361 & $46.7 \%$ & $4.3 \%$ \\
\hline Montana & 1,105 & 968 & 933 & 1,046 & 921 & 914 & 946 & 824 & 770 & 1,452 & $31.4 \%$ & $3.1 \%$ \\
\hline Nevada & 1,609 & 1,739 & 2,114 & 2,511 & 2,153 & 1,884 & 2,301 & 2,468 & 2,186 & 2,493 & $54.9 \%$ & $5.0 \%$ \\
\hline North Dakota & 704 & 403 & 672 & 577 & 1,312 & 1,255 & 642 & 740 & 935 & 1,002 & $42.5 \%$ & $4.0 \%$ \\
\hline Oregon & 780 & 795 & 668 & 844 & 834 & 761 & 1,060 & 925 & 1,048 & 1,152 & $47.6 \%$ & $4.4 \%$ \\
\hline South Dakota & 1,205 & 1,069 & 1,328 & 1,049 & 849 & 825 & 894 & 1,230 & 1,185 & 1,041 & $-13.6 \%$ & $-1.6 \%$ \\
\hline Utah & 1,665 & 1,632 & 1,614 & 1,857 & 2,305 & 2,758 & 2,945 & 2,815 & 2,624 & 2,749 & $65.1 \%$ & $5.7 \%$ \\
\hline Washington & 1,200 & 1,055 & 796 & 781 & 746 & 772 & 751 & 803 & 914 & 1,112 & $-7.4 \%$ & $-0.8 \%$ \\
\hline Wyoming & 1,611 & 1,898 & 1,928 & 1,546 & 1,543 & 1,583 & 1,167 & 1,915 & 1,628 & 2,016 & $25.2 \%$ & $2.5 \%$ \\
\hline Noridian Total & 1,286 & 1,228 & 1,193 & 1,226 & 1,227 & 1,208 & 1,284 & 1,300 & 1,364 & 1,492 & $16.1 \%$ & $1.7 \%$ \\
\hline PCPY & & $-4.5 \%$ & $-2.8 \%$ & $2.7 \%$ & $0.1 \%$ & $-1.5 \%$ & $6.3 \%$ & $1.3 \%$ & $4.9 \%$ & $9.4 \%$ & & \\
\hline Palmetto GBA & & & & & & & & & & & & \\
\hline
\end{tabular}


Pain Physician: May/June 2020 23:S129-S147

Appendix Table 2 con't. Utilization of facet joint interventions rate (episodes) by Medicare carrier and state.

\begin{tabular}{|c|c|c|c|c|c|c|c|c|c|c|c|c|}
\hline State name & R2009 & R2010 & R2011 & $\mathrm{R} 2012$ & R2013 & R2014 & R2015 & R2016 & R2017 & R2018 & Change & GM \\
\hline North Carolina & 1,376 & 1,289 & 1,334 & 1,509 & 1,336 & 1,301 & 1,510 & 1,508 & 1,624 & 1,613 & $17.2 \%$ & $1.8 \%$ \\
\hline South Carolina & 1,651 & 1,610 & 1,661 & 1,837 & 2,033 & 2,158 & 2,281 & 2,499 & 2,564 & 2,543 & $54.0 \%$ & $4.9 \%$ \\
\hline Virginia & 1,296 & 1,073 & 1,110 & 1,374 & 1,648 & 1,799 & 1,865 & 1,989 & 2,028 & 2,152 & $66.1 \%$ & $5.8 \%$ \\
\hline West Virginia & 1,230 & 1,231 & 1,378 & 1,367 & 1,480 & 1,944 & 1,911 & 2,121 & 2,393 & 1,959 & $59.2 \%$ & $5.3 \%$ \\
\hline Pllmetto Total & 1,393 & 1,284 & 1,338 & 1,522 & 1,587 & 1,689 & 1,815 & 1,919 & 2,015 & 2,005 & $43.9 \%$ & $4.1 \%$ \\
\hline PCY & & $-8 \%$ & $4 \%$ & $14 \%$ & $4 \%$ & $6 \%$ & $7 \%$ & $6 \%$ & $5 \%$ & $0 \%$ & & \\
\hline \multicolumn{13}{|l|}{ Novitas } \\
\hline Arkansas & 2,517 & 2,315 & 1,934 & 2,230 & 2,542 & 2,580 & 3,094 & 3,676 & 3,629 & 3,613 & $43.5 \%$ & $4.1 \%$ \\
\hline Colorado & 1,000 & 855 & 1,068 & 1,235 & 1,345 & 1,470 & 1,648 & 1,627 & 1,857 & 1,993 & $99.3 \%$ & $8.0 \%$ \\
\hline Delaware & 1,599 & 790 & 1,353 & 1,119 & 1,795 & 1,820 & 2,153 & 2,105 & 2,046 & 2,397 & $49.9 \%$ & $4.6 \%$ \\
\hline $\begin{array}{l}\text { District of } \\
\text { Columbia }\end{array}$ & 574 & 845 & 602 & 1,329 & 955 & 1,128 & 2,019 & 1,968 & 1,428 & 800 & $39.4 \%$ & $3.8 \%$ \\
\hline Louisiana & 1,019 & 1,366 & 1,559 & 1,588 & 1,697 & 1,841 & 2,103 & 2,060 & 2,211 & 2,042 & $100.4 \%$ & $8.0 \%$ \\
\hline Maryland & 1,712 & 1,493 & 1,678 & 1,815 & 1,888 & 2,220 & 2,465 & 2,254 & 2,063 & 2,416 & $41.1 \%$ & $3.9 \%$ \\
\hline Mississippi & 1,775 & 1,718 & 2,073 & 2,252 & 2,081 & 1,840 & 2,143 & 2,238 & 2,190 & 2,504 & $41.1 \%$ & $3.9 \%$ \\
\hline New Jersey & 964 & 1,094 & 1,128 & 1,187 & 1,289 & 1,562 & 1,767 & 1,722 & 1,715 & 1,864 & $93.2 \%$ & $7.6 \%$ \\
\hline New Mexico & 1,422 & 1,372 & 1,457 & 1,545 & 1,291 & 1,481 & 1,557 & 1,846 & 2,091 & 1,786 & $25.6 \%$ & $2.6 \%$ \\
\hline Oklahoma & 1,359 & 1,356 & 1,341 & 1,400 & 1,543 & 2,242 & 2,425 & 2,558 & 3,058 & 3,037 & $123.5 \%$ & $9.3 \%$ \\
\hline Pennsylvania & 1,040 & 991 & 934 & 956 & 1,104 & 1,151 & 1,280 & 1,355 & 1,479 & 1,491 & $43.3 \%$ & $4.1 \%$ \\
\hline Texas & 2,348 & 1,993 & 1,839 & 1,898 & 1,910 & 1,873 & 2,143 & 2,327 & 2,209 & 2,186 & $-6.9 \%$ & $-0.8 \%$ \\
\hline Novitas Total & 1,572 & 1,457 & 1,452 & 1,533 & 1,617 & 1,724 & 1,959 & 2,050 & 2,073 & 2,114 & $34.5 \%$ & $3.3 \%$ \\
\hline PCPY & & $-7.3 \%$ & $-0.4 \%$ & $5.6 \%$ & $5.5 \%$ & $6.6 \%$ & $13.6 \%$ & $4.7 \%$ & $1.1 \%$ & $2.0 \%$ & & \\
\hline \multicolumn{13}{|l|}{ WPS } \\
\hline Indiana & 1,634 & 1,694 & 1,706 & 1,686 & 1,658 & 1,938 & 2,090 & 2,187 & 1,960 & 1,995 & $22.1 \%$ & $2.2 \%$ \\
\hline Iowa & 797 & 897 & 945 & 911 & 1,064 & 1,114 & 1,267 & 1,308 & 1,458 & 1,427 & $78.9 \%$ & $6.7 \%$ \\
\hline Kansas & 950 & 1,021 & 992 & 1,196 & 988 & 913 & 1,115 & 1,285 & 1,204 & 1,380 & $45.3 \%$ & $4.2 \%$ \\
\hline Michigan & 2,681 & 2,390 & 2,358 & 2,577 & 2,734 & 2,975 & 2,813 & 2,599 & 2,354 & 1,929 & $-28.0 \%$ & $-3.6 \%$ \\
\hline Missouri & 1,449 & 1,515 & 1,424 & 1,567 & 1,723 & 1,775 & 1,742 & 1,612 & 1,669 & 1,843 & $27.1 \%$ & $2.7 \%$ \\
\hline Nebraska & 885 & 781 & 708 & 939 & 912 & 946 & 1,054 & 1,179 & 1,439 & 1,763 & $99.2 \%$ & $8.0 \%$ \\
\hline WPS Total & 1,755 & 1,696 & 1,670 & 1,798 & 1,880 & 2,033 & 2,041 & 1,983 & 1,887 & 1,816 & $3.4 \%$ & $0.4 \%$ \\
\hline PCY & & $-3.4 \%$ & $-1.6 \%$ & $7.7 \%$ & $4.6 \%$ & $8.1 \%$ & $0.4 \%$ & $-2.8 \%$ & $-4.8 \%$ & $-3.8 \%$ & & \\
\hline \multirow[t]{2}{*}{ USA Total } & 2,590 & 2,445 & 2,482 & 2,601 & 2,597 & 2,693 & 2,916 & 3,042 & 3,148 & 3,217 & $24.2 \%$ & $2.4 \%$ \\
\hline & & $-5.6 \%$ & $1.5 \%$ & $4.8 \%$ & $-0.1 \%$ & $3.7 \%$ & $8.3 \%$ & $4.3 \%$ & $3.5 \%$ & $2.2 \%$ & & \\
\hline
\end{tabular}

Rate - per 100,000 Medicare Beneficiaries 
Trends of Expenditures of Facet Joint Interventions in Medicare Population

Appendix Table 3. Utilization of lumbar facet joint injections rate per 100,000 Medicare beneficiaries (episodes) by 2016 Medicare carrier and state.

\begin{tabular}{|c|c|c|c|c|c|c|c|c|c|c|c|c|}
\hline State name & R2009 & R2010 & R2011 & R2012 & R2013 & R2014 & R2015 & R2016 & R2017 & R2018 & Change & GM \\
\hline \multicolumn{13}{|l|}{ Cahaba } \\
\hline Alabama & 969 & 1,228 & 1,224 & 1,347 & 1,141 & 1,127 & 1,098 & 1,264 & 1,050 & 1,006 & $3.8 \%$ & $0.4 \%$ \\
\hline Georgia & 1,099 & 1,107 & 1,087 & 1,175 & 1,131 & 1,154 & 1,246 & 1,235 & 1,116 & 1,210 & $10.1 \%$ & $1.1 \%$ \\
\hline Tennessee & 1,187 & 1,138 & 1,195 & 1,157 & 865 & 847 & 808 & 902 & 873 & 809 & $-31.8 \%$ & $-4.2 \%$ \\
\hline Cabha Total & 1,093 & 1,150 & 1,160 & 1,215 & 1,044 & 1,044 & 1,061 & 1,132 & 1,019 & 1,026 & $-6.2 \%$ & $-0.7 \%$ \\
\hline PCPY & & $5.2 \%$ & $0.9 \%$ & $4.7 \%$ & $-14.1 \%$ & $0.0 \%$ & $1.7 \%$ & $6.7 \%$ & $-10.0 \%$ & $0.7 \%$ & & \\
\hline \multicolumn{13}{|l|}{ CGS } \\
\hline Kentucky & 1,154 & 1,011 & 1,084 & 1,170 & 1,131 & 1,056 & 1,337 & 1,182 & 1,310 & 1,238 & $7.3 \%$ & $0.8 \%$ \\
\hline Ohio & 814 & 802 & 911 & 971 & 923 & 879 & 966 & 965 & 896 & 910 & $11.8 \%$ & $1.2 \%$ \\
\hline CGS Total & 911 & 861 & 961 & 1,028 & 983 & 930 & 1,073 & 1,027 & 1,014 & 1,003 & $10.2 \%$ & $1.1 \%$ \\
\hline PCPY & & $-5.4 \%$ & $11.5 \%$ & $7.0 \%$ & $-4.4 \%$ & $-5.4 \%$ & $15.3 \%$ & $-4.3 \%$ & $-1.2 \%$ & $-1.1 \%$ & & \\
\hline \multicolumn{13}{|l|}{ First Coast } \\
\hline Florida & 1,453 & 1,253 & 1,226 & 1,326 & 1,154 & 1,297 & 1,266 & 1,293 & 1,250 & 1,215 & $-16.4 \%$ & $-2.0 \%$ \\
\hline PCPY & & $-13.8 \%$ & $-2.2 \%$ & $8.2 \%$ & $-13.0 \%$ & $12.4 \%$ & $-2.4 \%$ & $2.2 \%$ & $-3.4 \%$ & $-2.8 \%$ & & \\
\hline \multicolumn{13}{|l|}{ NGS } \\
\hline Connecticut & 817 & 740 & 662 & 730 & 752 & 772 & 753 & 771 & 868 & 809 & $-1.0 \%$ & $-0.1 \%$ \\
\hline Illinois & 914 & 639 & 729 & 783 & 765 & 761 & 750 & 762 & 810 & 829 & $-9.4 \%$ & $-1.1 \%$ \\
\hline Maine & 648 & 680 & 650 & 673 & 743 & 722 & 973 & 751 & 720 & 719 & $10.8 \%$ & $1.1 \%$ \\
\hline Massachusetts & 929 & 899 & 937 & 1,032 & 1,063 & 1,097 & 1,148 & 1,010 & 1,060 & 1,038 & $11.6 \%$ & $1.2 \%$ \\
\hline Minnesota & 352 & 310 & 368 & 490 & 429 & 463 & 400 & 395 & 399 & 397 & $12.9 \%$ & $1.4 \%$ \\
\hline New Hampshire & 727 & 1,003 & 1,048 & 985 & 718 & 898 & 783 & 947 & 932 & 958 & $31.8 \%$ & $3.1 \%$ \\
\hline New York & 488 & 497 & 500 & 440 & 558 & 579 & 661 & 661 & 652 & 614 & $25.8 \%$ & $2.6 \%$ \\
\hline Rhode Island & 877 & 907 & 894 & 796 & 1,045 & 904 & 926 & 846 & 586 & 795 & $-9.3 \%$ & $-1.1 \%$ \\
\hline Vermont & 1,000 & 915 & 733 & 767 & 784 & 825 & 977 & 1,081 & 1,149 & 878 & $-12.3 \%$ & $-1.4 \%$ \\
\hline Wisconsin & 682 & 674 & 713 & 778 & 765 & 724 & 771 & 794 & 724 & 684 & $0.4 \%$ & $0.0 \%$ \\
\hline NGS Total & 682 & 623 & 649 & 670 & 705 & 716 & 752 & 740 & 746 & 727 & $6.7 \%$ & $0.7 \%$ \\
\hline PCPY & & $-8.7 \%$ & $4.2 \%$ & $3.3 \%$ & $5.1 \%$ & $1.7 \%$ & $5.0 \%$ & $-1.7 \%$ & $0.8 \%$ & $-2.5 \%$ & $-100.0 \%$ & \\
\hline \multicolumn{13}{|l|}{ Noridian } \\
\hline Alaska & 574 & 639 & 290 & 115 & 480 & 813 & 1,061 & 978 & 827 & 935 & $62.9 \%$ & $5.6 \%$ \\
\hline Arizona & 1,047 & 1,107 & 998 & 1,095 & 1,042 & 1,061 & 1,151 & 1,149 & 1,236 & 1,258 & $20.2 \%$ & $2.1 \%$ \\
\hline California & 836 & 682 & 687 & 664 & 611 & 552 & 574 & 581 & 588 & 630 & $-24.6 \%$ & $-3.1 \%$ \\
\hline Idaho & 496 & 409 & 387 & 659 & 548 & 578 & 548 & 688 & 692 & 749 & $51.2 \%$ & $4.7 \%$ \\
\hline Montana & 559 & 543 & 622 & 664 & 531 & 630 & 549 & 566 & 473 & 717 & $28.4 \%$ & $2.8 \%$ \\
\hline Nevada & 1,026 & 965 & 1,076 & 1,279 & 1,008 & 786 & 1,081 & 1,020 & 971 & 1,106 & $7.8 \%$ & $0.8 \%$ \\
\hline North Dakota & 389 & 238 & 254 & 361 & 773 & 460 & 260 & 421 & 484 & 642 & $65.1 \%$ & $5.7 \%$ \\
\hline Oregon & 478 & 470 & 372 & 447 & 404 & 430 & 589 & 509 & 587 & 638 & $33.4 \%$ & $3.3 \%$ \\
\hline South Dakota & 818 & 542 & 649 & 468 & 418 & 419 & 473 & 679 & 665 & 538 & $-34.2 \%$ & $-4.5 \%$ \\
\hline Utah & 935 & 728 & 752 & 915 & 1,110 & 1,430 & 1,347 & 1,135 & 1,145 & 1,123 & $20.2 \%$ & $2.1 \%$ \\
\hline Washington & 753 & 613 & 420 & 443 & 456 & 435 & 404 & 467 & 498 & 586 & $-22.1 \%$ & $-2.7 \%$ \\
\hline Wyoming & 972 & 899 & 903 & 975 & 1,013 & 981 & 821 & 1,136 & 824 & 1,168 & $20.2 \%$ & $2.1 \%$ \\
\hline Noridian Total & 814 & 702 & 670 & 695 & 656 & 630 & 666 & 673 & 689 & 744 & $-8.5 \%$ & $-1.0 \%$ \\
\hline PCPY & & $-13.7 \%$ & $-4.5 \%$ & $3.8 \%$ & $-5.6 \%$ & $-4.1 \%$ & $5.8 \%$ & $1.0 \%$ & $2.5 \%$ & $8.0 \%$ & & \\
\hline
\end{tabular}


Pain Physician: May/June 2020 23:S129-S147

Appendix Table 3 con't. Utilization of lumbar facet joint injections rate per 100,000 Medicare beneficiaries (episodes) by 2016

Medicare carrier and state.

\begin{tabular}{|c|c|c|c|c|c|c|c|c|c|c|c|c|}
\hline State name & R2009 & R2010 & R2011 & R2012 & R2013 & R2014 & R2015 & R2016 & R2017 & R2018 & Change & GM \\
\hline \multicolumn{13}{|l|}{ Palmetto GBA } \\
\hline North Carolina & 803 & 730 & 747 & 889 & 742 & 784 & 865 & 897 & 877 & 832 & $3.7 \%$ & $0.4 \%$ \\
\hline South Carolina & 1,085 & 998 & 1,026 & 1,101 & 1,260 & 1,330 & 1,340 & 1,456 & 1,449 & 1,382 & $27.4 \%$ & $2.7 \%$ \\
\hline Virginia & 867 & 726 & 731 & 851 & 1,025 & 1,029 & 1,100 & 1,113 & 1,101 & 1,186 & $36.9 \%$ & $3.5 \%$ \\
\hline West Virginia & 636 & 597 & 741 & 821 & 820 & 1,127 & 1,023 & 1,233 & 1,262 & 931 & $46.3 \%$ & $4.3 \%$ \\
\hline Palmetto Total & 862 & 770 & 799 & 914 & 941 & 1,004 & 1,050 & 1,111 & 1,101 & 1,065 & $23.5 \%$ & $2.4 \%$ \\
\hline PCPY & & $-11 \%$ & $4 \%$ & $14 \%$ & $3 \%$ & $7 \%$ & $5 \%$ & $6 \%$ & $-1 \%$ & $-3 \%$ & & \\
\hline \multicolumn{13}{|l|}{ Novitas } \\
\hline Arkansas & 1,172 & 1,099 & 967 & 1,173 & 1,219 & 1,111 & 1,365 & 1,500 & 1,519 & 1,424 & $21.5 \%$ & $2.2 \%$ \\
\hline Colorado & 538 & 522 & 560 & 671 & 686 & 694 & 808 & 784 & 743 & 810 & $50.6 \%$ & $4.7 \%$ \\
\hline Delaware & 1,034 & 522 & 963 & 750 & 1,106 & 1,076 & 1,253 & 1,318 & 1,384 & 1,323 & $27.9 \%$ & $2.8 \%$ \\
\hline Louisiana & 512 & 786 & 802 & 749 & 797 & 890 & 957 & 973 & 975 & 853 & $66.4 \%$ & $5.8 \%$ \\
\hline Maryland & 1,008 & 841 & 907 & 1,035 & 1,010 & 1,259 & 1,319 & 1,183 & 1,051 & 1,216 & $20.7 \%$ & $2.1 \%$ \\
\hline Mississippi & 1,041 & 990 & 1,200 & 1,343 & 1,187 & 1,018 & 1,196 & 1,228 & 1,265 & 1,303 & $25.1 \%$ & $2.5 \%$ \\
\hline New Jersey & 600 & 668 & 691 & 720 & 755 & 831 & 943 & 893 & 966 & 964 & $60.8 \%$ & $5.4 \%$ \\
\hline New Mexico & 698 & 759 & 819 & 879 & 796 & 740 & 773 & 880 & 937 & 903 & $29.4 \%$ & $2.9 \%$ \\
\hline Oklahoma & 858 & 756 & 667 & 751 & 775 & 1,072 & 1,300 & 1,318 & 1,407 & 1,481 & $72.5 \%$ & $6.2 \%$ \\
\hline Pennsylvania & 684 & 644 & 607 & 613 & 689 & 721 & 769 & 783 & 863 & 863 & $26.2 \%$ & $2.6 \%$ \\
\hline Texas & 1,324 & 1,036 & 964 & 993 & 922 & 902 & 1,044 & 1,074 & 960 & 933 & $-29.6 \%$ & $-3.8 \%$ \\
\hline Novitas Total & 906 & 816 & 806 & 854 & 854 & 890 & 1,006 & 1,015 & 1,003 & 1,000 & $10.4 \%$ & $1.1 \%$ \\
\hline PCPY & & $-9.9 \%$ & $-1.3 \%$ & $6.0 \%$ & $-0.1 \%$ & $4.2 \%$ & $13.1 \%$ & $0.9 \%$ & $-1.2 \%$ & $-0.3 \%$ & & \\
\hline Indiana & 952 & 1,034 & 974 & 1,106 & 942 & 1,052 & 1,179 & 1,259 & 1,107 & 1,123 & $18.0 \%$ & $1.9 \%$ \\
\hline Iowa & 551 & 595 & 558 & 527 & 635 & 611 & 657 & 773 & 792 & 794 & $44.0 \%$ & $4.1 \%$ \\
\hline Kansas & 574 & 605 & 578 & 808 & 596 & 530 & 549 & 620 & 675 & 787 & $37.3 \%$ & $3.6 \%$ \\
\hline Michigan & 1,639 & 1,497 & 1,459 & 1,563 & 1,624 & 1,857 & 1,706 & 1,443 & 1,256 & 969 & $-40.9 \%$ & $-5.7 \%$ \\
\hline Missouri & 767 & 866 & 812 & 934 & 975 & 997 & 961 & 947 & 952 & 1,082 & $41.1 \%$ & $3.9 \%$ \\
\hline Nebraska & 581 & 394 & 248 & 466 & 525 & 541 & 593 & 606 & 838 & 887 & $52.9 \%$ & $4.8 \%$ \\
\hline WPS Total & 1,048 & 1,035 & 985 & 1,103 & 1,099 & 1,199 & 1,175 & 1,115 & 1,040 & 985 & $-5.9 \%$ & $-0.7 \%$ \\
\hline PCY & & $-1.2 \%$ & $-4.8 \%$ & $12.0 \%$ & $-0.4 \%$ & $9.1 \%$ & $-2.0 \%$ & $-5.1 \%$ & $-6.7 \%$ & $-5.3 \%$ & & \\
\hline USA Total & 901 & 826 & 820 & 859 & 822 & 849 & 896 & 907 & 911 & 906 & $0.6 \%$ & $0.1 \%$ \\
\hline PCPY & & $-8.3 \%$ & $-0.7 \%$ & $4.8 \%$ & $-4.4 \%$ & $3.4 \%$ & $5.5 \%$ & $1.2 \%$ & $0.5 \%$ & $-0.6 \%$ & & \\
\hline
\end{tabular}


Trends of Expenditures of Facet Joint Interventions in Medicare Population

Appendix Table 4. Utilization of lumbar facet neurolysis rate per 100,000 Medicare beneficiaries (episodes) by 2016 Medicare carrier and state.

\begin{tabular}{|c|c|c|c|c|c|c|c|c|c|c|c|c|}
\hline State name & R2009 & R2010 & R2011 & R2012 & R2013 & R2014 & R2015 & R2016 & R2017 & R2018 & Change & GM \\
\hline \multicolumn{13}{|l|}{ Cahaba } \\
\hline Alabama & 111 & 144 & 160 & 231 & 221 & 254 & 257 & 362 & 341 & 381 & $243.2 \%$ & $14.7 \%$ \\
\hline Georgia & 390 & 447 & 405 & 443 & 438 & 494 & 584 & 578 & 669 & 715 & $83.1 \%$ & $7.0 \%$ \\
\hline Tennessee & 223 & 161 & 228 & 306 & 262 & 259 & 329 & 392 & 437 & 433 & $94.2 \%$ & $7.7 \%$ \\
\hline Cahaba Total & 258 & 269 & 280 & 341 & 321 & 352 & 413 & 460 & 508 & 537 & $108.1 \%$ & $8.5 \%$ \\
\hline PCPY & & $4.2 \%$ & $4.1 \%$ & $21.8 \%$ & $-5.7 \%$ & $9.6 \%$ & $17.4 \%$ & $11.2 \%$ & $10.5 \%$ & $5.7 \%$ & & \\
\hline \multicolumn{13}{|l|}{ CGS } \\
\hline Kentucky & 336 & 374 & 402 & 509 & 501 & 517 & 649 & 640 & 799 & 853 & $153.5 \%$ & $10.9 \%$ \\
\hline Ohio & 199 & 256 & 309 & 370 & 373 & 420 & 422 & 473 & 453 & 502 & $152.3 \%$ & $10.8 \%$ \\
\hline \multirow[t]{2}{*}{ CGS Total } & 238 & 289 & 336 & 410 & 410 & 448 & 487 & 521 & 551 & 602 & $152.8 \%$ & $10.9 \%$ \\
\hline & & $21.6 \%$ & $16.0 \%$ & $22.2 \%$ & $0.0 \%$ & $9.2 \%$ & $8.8 \%$ & $6.9 \%$ & $5.8 \%$ & $9.1 \%$ & & \\
\hline \multicolumn{13}{|l|}{ First Coast } \\
\hline Florida & 392 & 334 & 329 & 387 & 378 & 470 & 488 & 580 & 617 & 627 & $60.1 \%$ & $5.4 \%$ \\
\hline PCPY & & $-14.6 \%$ & $-1.6 \%$ & $17.7 \%$ & $-2.5 \%$ & $24.5 \%$ & $3.7 \%$ & $18.9 \%$ & $6.4 \%$ & $1.6 \%$ & & \\
\hline \multicolumn{13}{|l|}{ NGS } \\
\hline Connecticut & 104 & 137 & 135 & 174 & 157 & 161 & 253 & 225 & 250 & 359 & $245.8 \%$ & $14.8 \%$ \\
\hline Illinois & 220 & 194 & 231 & 286 & 310 & 326 & 366 & 382 & 425 & 415 & $88.3 \%$ & $7.3 \%$ \\
\hline Maine & 239 & 159 & 214 & 152 & 241 & 241 & 302 & 222 & 279 & 205 & $-14.2 \%$ & $-1.7 \%$ \\
\hline Massachusetts & 196 & 213 & 219 & 272 & 349 & 272 & 381 & 355 & 318 & 391 & $99.1 \%$ & $8.0 \%$ \\
\hline Minnesota & 141 & 84 & 95 & 173 & 152 & 178 & 119 & 171 & 203 & 173 & $22.6 \%$ & $2.3 \%$ \\
\hline New Hampshire & 340 & 394 & 629 & 458 & 257 & 363 & 400 & 466 & 498 & 558 & $64.0 \%$ & $5.6 \%$ \\
\hline New York & 155 & 141 & 151 & 170 & 222 & 229 & 269 & 294 & 253 & 309 & $99.0 \%$ & $7.9 \%$ \\
\hline Rhode Island & 166 & 131 & 86 & 53 & 110 & 22 & 74 & 118 & 104 & 157 & $-5.6 \%$ & $-0.6 \%$ \\
\hline Vermont & 371 & 287 & 332 & 307 & 434 & 501 & 394 & 563 & 531 & 418 & $12.8 \%$ & $1.3 \%$ \\
\hline Wisconsin & 229 & 222 & 204 & 306 & 301 & 313 & 353 & 394 & 387 & 417 & $82.1 \%$ & $6.9 \%$ \\
\hline NGS Total & 186 & 172 & 190 & 226 & 255 & 257 & 298 & 317 & 313 & 344 & $84.6 \%$ & $7.0 \%$ \\
\hline PCPY & & $-7.9 \%$ & $10.6 \%$ & $19.2 \%$ & $12.7 \%$ & $0.7 \%$ & $16.1 \%$ & $6.1 \%$ & $-1.1 \%$ & $9.9 \%$ & & \\
\hline \multicolumn{13}{|l|}{ Noridain } \\
\hline Alaska & 128 & 61 & 174 & 260 & 226 & 325 & 440 & 477 & 479 & 520 & $307.3 \%$ & $16.9 \%$ \\
\hline Arizona & 334 & 434 & 436 & 483 & 570 & 564 & 631 & 668 & 799 & 989 & $196.7 \%$ & $12.8 \%$ \\
\hline California & 151 & 182 & 196 & 194 & 203 & 223 & 216 & 233 & 248 & 258 & $70.9 \%$ & $6.1 \%$ \\
\hline Idaho & 189 & 218 & 194 & 222 & 211 & 323 & 339 & 326 & 333 & 300 & $58.4 \%$ & $5.2 \%$ \\
\hline Montana & 316 & 236 & 150 & 124 & 173 & 126 & 224 & 149 & 158 & 314 & $-0.6 \%$ & $-0.1 \%$ \\
\hline Nevada & 257 & 275 & 414 & 542 & 568 & 439 & 494 & 671 & 609 & 723 & $181.8 \%$ & $12.2 \%$ \\
\hline North Dakota & 167 & 110 & 182 & 144 & 234 & 389 & 278 & 236 & 274 & 204 & $22.2 \%$ & $2.3 \%$ \\
\hline Oregon & 139 & 187 & 153 & 174 & 180 & 178 & 215 & 204 & 233 & 251 & $79.9 \%$ & $6.7 \%$ \\
\hline South Dakota & 193 & 161 & 260 & 255 & 111 & 176 & 145 & 282 & 230 & 269 & $39.2 \%$ & $3.7 \%$ \\
\hline Utah & 453 & 452 & 465 & 594 & 720 & 915 & 1,139 & 921 & 941 & 968 & $113.8 \%$ & $8.8 \%$ \\
\hline Washington & 200 & 185 & 129 & 130 & 123 & 157 & 138 & 151 & 194 & 267 & $33.0 \%$ & $3.2 \%$ \\
\hline Wyoming & 486 & 475 & 464 & 262 & 230 & 89 & 216 & 358 & 451 & 490 & $0.8 \%$ & $0.1 \%$ \\
\hline Noridian Total & 197 & 225 & 231 & 245 & 264 & 283 & 300 & 315 & 345 & 390 & $97.8 \%$ & $7.9 \%$ \\
\hline PCPY & & $14.1 \%$ & $2.7 \%$ & $5.9 \%$ & $7.7 \%$ & $7.4 \%$ & $5.9 \%$ & $5.1 \%$ & $9.4 \%$ & $13.1 \%$ & & \\
\hline
\end{tabular}


Pain Physician: May/June 2020 23:S129-S147

Appendix Table 4 con't. Utilization of lumbar facet neurolysis rate per 100,000 Medicare beneficiaries (episodes) by 2016 Medicare carrier and state.

\begin{tabular}{|c|c|c|c|c|c|c|c|c|c|c|c|c|}
\hline State name & R2009 & R2010 & $\begin{array}{l}\text { R2011 } \\
\end{array}$ & R2012 & R2013 & R2014 & R2015 & $\overline{\mathrm{R} 2016}$ & R2017 & R2018 & Change & GM \\
\hline \multicolumn{13}{|l|}{ Palmetto GBA } \\
\hline North Carolina & 331 & 314 & 350 & 318 & 305 & 276 & 349 & 356 & 430 & 415 & $25.2 \%$ & $2.5 \%$ \\
\hline South Carolina & 251 & 313 & 333 & 336 & 431 & 420 & 513 & 582 & 624 & 625 & $148.8 \%$ & $10.7 \%$ \\
\hline Virginia & 198 & 195 & 174 & 228 & 306 & 348 & 423 & 440 & 566 & 585 & $195.1 \%$ & $12.8 \%$ \\
\hline West Virginia & 292 & 299 & 290 & 230 & 285 & 364 & 359 & 456 & 594 & 544 & $86.5 \%$ & $7.2 \%$ \\
\hline Palmetto Total & 271 & 276 & 287 & 286 & 330 & 336 & 406 & 438 & 527 & 522 & $92.9 \%$ & $7.6 \%$ \\
\hline PCPY & & $2 \%$ & $4 \%$ & $-1 \%$ & $16 \%$ & $2 \%$ & $21 \%$ & $8 \%$ & $20 \%$ & $-1 \%$ & & \\
\hline \multicolumn{13}{|l|}{ Novitas } \\
\hline Arkansas & 688 & 696 & 557 & 623 & 791 & 809 & 1,034 & 1,271 & 1,331 & 1,354 & $96.8 \%$ & $7.8 \%$ \\
\hline Colorado & 126 & 118 & 172 & 264 & 260 & 304 & 388 & 392 & 536 & 536 & $324.3 \%$ & $17.4 \%$ \\
\hline Delaware & 207 & 80 & 182 & 114 & 209 & 165 & 330 & 410 & 393 & 577 & $178.9 \%$ & $12.1 \%$ \\
\hline $\begin{array}{l}\text { District of } \\
\text { Columbia }\end{array}$ & 52 & 179 & 125 & 320 & 393 & 495 & 646 & 1,108 & 483 & 259 & $397.2 \%$ & $19.5 \%$ \\
\hline Louisiana & 268 & 300 & 424 & 465 & 456 & 547 & 672 & 673 & 734 & 714 & $166.2 \%$ & $11.5 \%$ \\
\hline Maryland & 377 & 319 & 421 & 459 & 542 & 478 & 668 & 621 & 606 & 704 & $86.7 \%$ & $7.2 \%$ \\
\hline Mississippi & 238 & 298 & 391 & 395 & 451 & 367 & 396 & 400 & 445 & 660 & $177.6 \%$ & $12.0 \%$ \\
\hline New Jersey & 163 & 181 & 181 & 212 & 249 & 344 & 422 & 428 & 394 & 461 & $183.4 \%$ & $12.3 \%$ \\
\hline New Mexico & 197 & 274 & 360 & 315 & 289 & 373 & 372 & 553 & 519 & 493 & $149.6 \%$ & $10.7 \%$ \\
\hline Oklahoma & 213 & 268 & 355 & 320 & 400 & 615 & 543 & 610 & 864 & 900 & $322.5 \%$ & $17.4 \%$ \\
\hline Pennsylvania & 144 & 162 & 161 & 161 & 204 & 230 & 281 & 285 & 298 & 324 & $125.3 \%$ & $9.4 \%$ \\
\hline Texas & 384 & 417 & 381 & 440 & 505 & 523 & 567 & 675 & 702 & 666 & $73.4 \%$ & $6.3 \%$ \\
\hline Novitas Total & 272 & 293 & 307 & 339 & 393 & 430 & 500 & 554 & 586 & 607 & $123.2 \%$ & $9.3 \%$ \\
\hline PCPY & & $7.6 \%$ & $4.9 \%$ & $10.4 \%$ & $15.9 \%$ & $9.5 \%$ & $16.3 \%$ & $10.9 \%$ & $5.6 \%$ & $3.7 \%$ & & \\
\hline \multicolumn{13}{|l|}{ WPS } \\
\hline Indiana & 272 & 251 & 312 & 237 & 308 & 354 & 431 & 455 & 453 & 449 & $65.0 \%$ & $5.7 \%$ \\
\hline Iowa & 121 & 201 & 203 & 207 & 244 & 228 & 305 & 276 & 435 & 359 & $196.4 \%$ & $12.8 \%$ \\
\hline Kansas & 197 & 180 & 219 & 161 & 163 & 190 & 228 & 250 & 314 & 283 & $43.3 \%$ & $4.1 \%$ \\
\hline Michigan & 269 & 327 & 275 & 353 & 379 & 388 & 406 & 406 & 428 & 407 & $51.4 \%$ & $4.7 \%$ \\
\hline Missouri & 209 & 239 & 262 & 296 & 328 & 349 & 336 & 292 & 360 & 380 & $81.8 \%$ & $6.9 \%$ \\
\hline Nebraska & 138 & 158 & 198 & 209 & 221 & 216 & 283 & 261 & 328 & 444 & $221.9 \%$ & $13.9 \%$ \\
\hline WPS Total & 228 & 257 & 263 & 277 & 312 & 330 & 364 & 358 & 404 & 397 & $74.2 \%$ & $6.4 \%$ \\
\hline PCY & & $13.0 \%$ & $2.2 \%$ & $5.3 \%$ & $12.8 \%$ & $5.6 \%$ & $10.5 \%$ & $-1.8 \%$ & $13.0 \%$ & $-1.9 \%$ & & \\
\hline \multirow[t]{2}{*}{ USA Total } & 240 & 250 & 262 & 286 & 308 & 332 & 375 & 411 & 452 & 475 & $97.7 \%$ & $7.9 \%$ \\
\hline & & $4.1 \%$ & $4.5 \%$ & $9.5 \%$ & $7.4 \%$ & $8.0 \%$ & $12.8 \%$ & $9.5 \%$ & $10.2 \%$ & $5.1 \%$ & & \\
\hline
\end{tabular}


Trends of Expenditures of Facet Joint Interventions in Medicare Population

Appendix Table 5. Utilization of cervical/thoracic facet joint injections rate per 100,000 Medicare beneficiaries (episodes) by 2016

Medicare carrier and state.

\begin{tabular}{|c|c|c|c|c|c|c|c|c|c|c|c|c|}
\hline State name & R2009 & R2010 & R2011 & R2012 & R2013 & R2014 & R2015 & R2016 & R2017 & R2018 & Change & GM \\
\hline \multicolumn{13}{|l|}{ Cahaba } \\
\hline Alabama & 333 & 402 & 336 & 392 & 371 & 363 & 337 & 318 & 286 & 315 & $-5.5 \%$ & $-0.6 \%$ \\
\hline Georgia & 310 & 274 & 345 & 423 & 397 & 358 & 373 & 423 & 387 & 364 & $17.4 \%$ & $1.8 \%$ \\
\hline Tennessee & 328 & 357 & 318 & 281 & 255 & 194 & 181 & 248 & 235 & 267 & $-18.5 \%$ & $-2.2 \%$ \\
\hline Cahaba Total & 322 & 336 & 334 & 367 & 342 & 304 & 299 & 337 & 311 & 320 & $-0.8 \%$ & $-0.1 \%$ \\
\hline PCPY & & $4.4 \%$ & $-0.8 \%$ & $10.1 \%$ & $-6.8 \%$ & $-11.1 \%$ & $-1.7 \%$ & $12.8 \%$ & $-7.9 \%$ & $2.9 \%$ & & \\
\hline \multicolumn{13}{|l|}{ CGS } \\
\hline Kentucky & 441 & 366 & 409 & 401 & 440 & 365 & 341 & 440 & 353 & 373 & $-15.6 \%$ & $-1.9 \%$ \\
\hline Ohio & 175 & 210 & 219 & 221 & 285 & 251 & 287 & 306 & 269 & 224 & $27.7 \%$ & $2.8 \%$ \\
\hline CGS Total & 251 & 255 & 274 & 273 & 330 & 284 & 303 & 345 & 293 & 266 & $6.1 \%$ & $0.7 \%$ \\
\hline PCPY & & $1.5 \%$ & $7.4 \%$ & $-0.4 \%$ & $20.9 \%$ & $-13.9 \%$ & $6.6 \%$ & $13.9 \%$ & $-14.9 \%$ & $-9.2 \%$ & & \\
\hline \multicolumn{13}{|l|}{ First Coast } \\
\hline Florida & 404 & 410 & 472 & 468 & 434 & 471 & 499 & 493 & 448 & 457 & $13.3 \%$ & $1.4 \%$ \\
\hline PCPY & & $1.4 \%$ & $15.3 \%$ & $-1.0 \%$ & $-7.2 \%$ & $8.5 \%$ & $6.1 \%$ & $-1.3 \%$ & $-9.1 \%$ & $2.1 \%$ & & \\
\hline \multicolumn{13}{|l|}{ NGS } \\
\hline Connecticut & 115 & 197 & 146 & 113 & 178 & 182 & 179 & 124 & 168 & 186 & $61.9 \%$ & $5.5 \%$ \\
\hline Illinois & 221 & 158 & 205 & 260 & 184 & 201 & 231 & 214 & 249 & 210 & $-5.3 \%$ & $-0.6 \%$ \\
\hline Maine & 185 & 68 & 185 & 246 & 219 & 488 & 409 & 411 & 298 & 248 & $33.6 \%$ & $3.3 \%$ \\
\hline Massachusetts & 177 & 217 & 238 & 288 & 224 & 254 & 182 & 205 & 281 & 268 & $51.5 \%$ & $4.7 \%$ \\
\hline Minnesota & 125 & 112 & 162 & 132 & 162 & 130 & 211 & 138 & 101 & 157 & $25.1 \%$ & $2.5 \%$ \\
\hline New Hampshire & 156 & 260 & 218 & 389 & 308 & 255 & 312 & 376 & 249 & 179 & $14.6 \%$ & $1.5 \%$ \\
\hline New York & 170 & 127 & 128 & 115 & 156 & 168 & 190 & 199 & 192 & 194 & $13.9 \%$ & $1.5 \%$ \\
\hline Rhode Island & 255 & 164 & 291 & 106 & 33 & 118 & 42 & 138 & 113 & 148 & $-42.0 \%$ & $-5.9 \%$ \\
\hline Vermont & 204 & 108 & 140 & 187 & 234 & 437 & 284 & 167 & 201 & 195 & $-4.3 \%$ & $-0.5 \%$ \\
\hline Wisconsin & 164 & 162 & 223 & 200 & 204 & 186 & 168 & 202 & 209 & 215 & $31.5 \%$ & $3.1 \%$ \\
\hline NGS Total & 176 & 153 & 178 & 187 & 181 & 199 & 204 & 202 & 210 & 205 & $16.5 \%$ & $1.7 \%$ \\
\hline PCPY & & $-13.0 \%$ & $16.5 \%$ & $5.0 \%$ & $-3.2 \%$ & $9.9 \%$ & $2.8 \%$ & $-1.0 \%$ & $3.7 \%$ & $-2.4 \%$ & $-99.9 \%$ & \\
\hline \multicolumn{13}{|l|}{ Noridian } \\
\hline Alaska & 447 & 274 & 203 & 115 & 198 & 136 & 233 & 238 & 283 & 374 & $-16.2 \%$ & $-1.9 \%$ \\
\hline Arizona & 265 & 411 & 345 & 409 & 441 & 371 & 425 & 400 & 536 & 497 & $87.9 \%$ & $7.3 \%$ \\
\hline California & 241 & 232 & 203 & 174 & 189 & 202 & 206 & 185 & 181 & 189 & $-21.5 \%$ & $-2.6 \%$ \\
\hline Idaho & 135 & 131 & 152 & 148 & 258 & 158 & 123 & 191 & 189 & 237 & $75.6 \%$ & $6.5 \%$ \\
\hline Montana & 170 & 165 & 127 & 202 & 173 & 126 & 102 & 99 & 111 & 323 & $89.8 \%$ & $7.4 \%$ \\
\hline Nevada & 262 & 365 & 430 & 516 & 399 & 454 & 484 & 464 & 301 & 414 & $57.9 \%$ & $5.2 \%$ \\
\hline North Dakota & 56 & 18 & 164 & - & 234 & 212 & 69 & 17 & 64 & 125 & $125.6 \%$ & $9.5 \%$ \\
\hline Oregon & 136 & 109 & 116 & 177 & 186 & 110 & 185 & 162 & 178 & 198 & $45.7 \%$ & $4.3 \%$ \\
\hline South Dakota & 134 & 351 & 332 & 269 & 265 & 203 & 197 & 179 & 230 & 129 & $-3.9 \%$ & $-0.4 \%$ \\
\hline Utah & 153 & 346 & 246 & 254 & 324 & 210 & 331 & 492 & 317 & 368 & $139.6 \%$ & $10.2 \%$ \\
\hline Washington & 200 & 202 & 195 & 183 & 140 & 153 & 170 & 139 & 174 & 195 & $-2.5 \%$ & $-0.3 \%$ \\
\hline Wyoming & 153 & 400 & 464 & 238 & 230 & 446 & 130 & 274 & 275 & 283 & $84.2 \%$ & $7.0 \%$ \\
\hline Noridian Total & 222 & 245 & 223 & 216 & 228 & 218 & 234 & 221 & 229 & 245 & $10.6 \%$ & $1.1 \%$ \\
\hline PCPY & & $10.6 \%$ & $-9.2 \%$ & $-2.9 \%$ & $5.2 \%$ & $-4.1 \%$ & $7.1 \%$ & $-5.4 \%$ & $3.5 \%$ & $7.1 \%$ & & \\
\hline
\end{tabular}


Pain Physician: May/June 2020 23:S129-S147

Appendix Table 5 con't. Utilization of cervical/thoracic facet joint injections rate per 100,000 Medicare beneficiaries (episodes) by 2016 Medicare carrier and state.

\begin{tabular}{|c|c|c|c|c|c|c|c|c|c|c|c|c|}
\hline State name & R2009 & R2010 & R2011 & R2012 & R2013 & R2014 & R2015 & R2016 & R2017 & R2018 & Change & GM \\
\hline \multicolumn{13}{|l|}{ Palmetto GBA } \\
\hline North Carolina & 193 & 173 & 187 & 242 & 239 & 209 & 218 & 197 & 219 & 253 & $30.6 \%$ & $3.0 \%$ \\
\hline South Carolina & 278 & 256 & 245 & 334 & 276 & 326 & 330 & 366 & 364 & 392 & $41.1 \%$ & $3.9 \%$ \\
\hline Virginia & 196 & 130 & 167 & 248 & 265 & 326 & 279 & 334 & 258 & 290 & $47.5 \%$ & $4.4 \%$ \\
\hline West Virginia & 254 & 272 & 264 & 235 & 265 & 335 & 407 & 317 & 397 & 350 & $37.6 \%$ & $3.6 \%$ \\
\hline Palmetto Total & 218 & 187 & 200 & 262 & 257 & 280 & 277 & 285 & 278 & 302 & $38.8 \%$ & $3.7 \%$ \\
\hline PCPY & & $-14 \%$ & $7 \%$ & $31 \%$ & $-2 \%$ & $9 \%$ & $-1 \%$ & $3 \%$ & $-2 \%$ & $9 \%$ & & \\
\hline \multicolumn{13}{|l|}{ Novitas } \\
\hline Arkansas & 411 & 320 & 251 & 290 & 316 & 347 & 348 & 528 & 445 & 497 & $20.9 \%$ & $2.1 \%$ \\
\hline Colorado & 256 & 163 & 246 & 243 & 292 & 329 & 310 & 278 & 394 & 418 & $63.3 \%$ & $5.6 \%$ \\
\hline Delaware & 345 & 174 & 156 & 216 & 467 & 485 & 490 & 244 & 227 & 388 & $12.5 \%$ & $1.3 \%$ \\
\hline $\begin{array}{l}\text { District of } \\
\text { Columbia }\end{array}$ & 130 & 128 & - & 123 & 28 & - & 242 & 90 & 110 & 65 & $-50.3 \%$ & $-7.5 \%$ \\
\hline Louisiana & 164 & 195 & 248 & 276 & 322 & 276 & 309 & 280 & 331 & 302 & $84.0 \%$ & $7.0 \%$ \\
\hline Maryland & 277 & 265 & 280 & 249 & 265 & 384 & 341 & 320 & 293 & 372 & $34.0 \%$ & $3.3 \%$ \\
\hline Mississippi & 398 & 362 & 407 & 410 & 391 & 404 & 450 & 471 & 376 & 430 & $8.1 \%$ & $0.9 \%$ \\
\hline New Jersey & 170 & 203 & 220 & 212 & 232 & 308 & 300 & 327 & 268 & 331 & $94.2 \%$ & $7.7 \%$ \\
\hline New Mexico & 395 & 300 & 223 & 236 & 175 & 297 & 333 & 241 & 403 & 288 & $-27.1 \%$ & $-3.5 \%$ \\
\hline Oklahoma & 247 & 275 & 257 & 252 & 252 & 423 & 415 & 440 & 552 & 448 & $81.8 \%$ & $6.9 \%$ \\
\hline Pennsylvania & 186 & 157 & 144 & 140 & 173 & 160 & 184 & 223 & 270 & 239 & $28.9 \%$ & $2.9 \%$ \\
\hline Texas & 541 & 434 & 390 & 340 & 330 & 323 & 379 & 376 & 351 & 364 & $-32.8 \%$ & $-4.3 \%$ \\
\hline Novitas Total & 322 & 278 & 269 & 256 & 272 & 300 & 322 & 331 & 337 & 345 & $7.2 \%$ & $0.8 \%$ \\
\hline PCPY & & $-13.8 \%$ & $-3.1 \%$ & $-4.6 \%$ & $6.2 \%$ & $10.1 \%$ & $7.5 \%$ & $2.7 \%$ & $1.7 \%$ & $2.5 \%$ & & \\
\hline \multicolumn{13}{|l|}{ WPS } \\
\hline Indiana & 337 & 340 & 331 & 292 & 339 & 425 & 381 & 382 & 322 & 339 & $0.5 \%$ & $0.1 \%$ \\
\hline Iowa & 102 & 70 & 122 & 128 & 137 & 206 & 220 & 199 & 167 & 193 & $89.6 \%$ & $7.4 \%$ \\
\hline Kansas & 132 & 166 & 123 & 187 & 185 & 155 & 266 & 345 & 184 & 237 & $80.1 \%$ & $6.8 \%$ \\
\hline Michigan & 725 & 483 & 558 & 569 & 630 & 645 & 593 & 651 & 581 & 433 & $-40.3 \%$ & $-5.6 \%$ \\
\hline Missouri & 406 & 360 & 307 & 298 & 365 & 353 & 340 & 313 & 282 & 315 & $-22.3 \%$ & $-2.8 \%$ \\
\hline Nebraska & 131 & 201 & 198 & 202 & 131 & 155 & 151 & 255 & 194 & 308 & $135.5 \%$ & $10.0 \%$ \\
\hline WPS Total & 426 & 341 & 355 & 356 & 399 & 425 & 406 & 431 & 367 & 340 & $-20.2 \%$ & $-2.5 \%$ \\
\hline PCY & & $-20.1 \%$ & $4.3 \%$ & $0.2 \%$ & $12.0 \%$ & $6.7 \%$ & $-4.4 \%$ & $5.9 \%$ & $-14.7 \%$ & $-7.3 \%$ & & \\
\hline USA Total & 278 & 257 & 262 & 262 & 265 & 275 & 285 & 293 & 290 & 293 & $5.6 \%$ & $0.6 \%$ \\
\hline PCPY & & $-7.5 \%$ & $2.2 \%$ & $-0.2 \%$ & $1.4 \%$ & $3.5 \%$ & $3.8 \%$ & $2.9 \%$ & $-1.2 \%$ & $1.2 \%$ & & \\
\hline
\end{tabular}


Trends of Expenditures of Facet Joint Interventions in Medicare Population

Appendix Table 6. Utilization of cervical/thoracic facet neurolysis rate per 100,000 Medicare beneficiaries (episodes) by 2016 Medicare carrier and state.

\begin{tabular}{|c|c|c|c|c|c|c|c|c|c|c|c|c|}
\hline State name & R2009 & R2010 & R2011 & R2012 & R2013 & R2014 & R2015 & R2016 & R2017 & R2018 & Change & GM \\
\hline \multicolumn{13}{|l|}{ Cahaba } \\
\hline Alabama & 24 & 50 & 46 & 18 & 42 & 65 & 61 & 81 & 77 & 91 & $278.5 \%$ & $15.9 \%$ \\
\hline Georgia & 84 & 95 & 116 & 135 & 143 & 154 & 157 & 158 & 197 & 178 & $112.2 \%$ & $8.7 \%$ \\
\hline Tennessee & 29 & 45 & 50 & 41 & 50 & 40 & 72 & 96 & 131 & 92 & $216.5 \%$ & $13.7 \%$ \\
\hline Cabha Total & 49 & 66 & 75 & 73 & 85 & 93 & 104 & 117 & 145 & 128 & $159.6 \%$ & $11.2 \%$ \\
\hline PCPY & & $34.9 \%$ & $13.0 \%$ & $-3.2 \%$ & $17.5 \%$ & $8.7 \%$ & $12.1 \%$ & $12.9 \%$ & $23.5 \%$ & $-11.8 \%$ & & \\
\hline \multicolumn{13}{|l|}{ CGS } \\
\hline Kentucky & 86 & 68 & 103 & 111 & 141 & 101 & 104 & 165 & 201 & 221 & $157.1 \%$ & $11.1 \%$ \\
\hline Ohio & 49 & 42 & 48 & 43 & 100 & 75 & 92 & 108 & 101 & 105 & $114.3 \%$ & $8.8 \%$ \\
\hline \multirow[t]{2}{*}{ CGS Total } & 60 & 50 & 63 & 62 & 112 & 83 & 95 & 124 & 130 & 138 & $131.9 \%$ & $9.8 \%$ \\
\hline & & $-16.9 \%$ & $27.9 \%$ & $-2.0 \%$ & $79.7 \%$ & $-25.9 \%$ & $15.3 \%$ & $29.9 \%$ & $4.7 \%$ & $6.7 \%$ & & \\
\hline \multicolumn{13}{|l|}{ FirstCoast } \\
\hline Florida & 98 & 88 & 96 & 128 & 101 & 122 & 146 & 175 & 152 & 186 & $90.3 \%$ & $7.4 \%$ \\
\hline PCPY & & $-10.4 \%$ & $9.0 \%$ & $33.4 \%$ & $-20.9 \%$ & $21.3 \%$ & $19.6 \%$ & $19.8 \%$ & $-13.5 \%$ & $22.7 \%$ & & \\
\hline \multicolumn{13}{|l|}{ NGS } \\
\hline Connecticut & 29 & 4 & 28 & 34 & 17 & 27 & 47 & 44 & 64 & 51 & $77.6 \%$ & $6.6 \%$ \\
\hline Illinois & 40 & 27 & 43 & 61 & 52 & 58 & 70 & 53 & 63 & 75 & $87.6 \%$ & $7.2 \%$ \\
\hline Maine & 15 & 23 & 7 & 58 & 50 & 55 & 34 & 85 & 68 & 66 & $330.2 \%$ & $17.6 \%$ \\
\hline Massachusetts & 38 & 21 & 31 & 49 & 61 & 61 & 44 & 38 & 55 & 71 & $83.2 \%$ & $7.0 \%$ \\
\hline Minnesota & 60 & 38 & 55 & 59 & 52 & 49 & 83 & 61 & 58 & 82 & $37.2 \%$ & $3.6 \%$ \\
\hline New Hampshire & 9 & 36 & 61 & 130 & 86 & 99 & 112 & 135 & 128 & 110 & $1098.5 \%$ & $31.8 \%$ \\
\hline New York & 36 & 24 & 26 & 29 & 30 & 52 & 51 & 62 & 60 & 58 & $60.3 \%$ & $5.4 \%$ \\
\hline Rhode Island & 89 & 186 & 22 & - & - & - & - & 20 & 28 & 46 & $-47.9 \%$ & $-7.0 \%$ \\
\hline Vermont & 37 & 233 & 52 & 34 & 50 & 65 & 63 & 107 & 57 & 98 & $163.2 \%$ & $11.4 \%$ \\
\hline Wisconsin & 47 & 33 & 52 & 76 & 63 & 71 & 71 & 80 & 90 & 100 & $111.9 \%$ & $8.7 \%$ \\
\hline NGS Total & 40 & 31 & 36 & 49 & 44 & 55 & 59 & 60 & 65 & 71 & $78.8 \%$ & $6.7 \%$ \\
\hline PCPY & & $-21.1 \%$ & $14.2 \%$ & $35.6 \%$ & $-8.3 \%$ & $23.8 \%$ & $7.3 \%$ & $2.3 \%$ & $6.8 \%$ & $10.0 \%$ & $-98.9 \%$ & \\
\hline \multicolumn{13}{|l|}{ Noridian } \\
\hline Alaska & 32 & - & 29 & - & 85 & 108 & 26 & 119 & 44 & 104 & $225.8 \%$ & $14.0 \%$ \\
\hline Arizona & 78 & 151 & 145 & 166 & 195 & 160 & 220 & 211 & 269 & 311 & $300.2 \%$ & $16.7 \%$ \\
\hline California & 45 & 34 & 51 & 54 & 59 & 56 & 58 & 57 & 66 & 71 & $58.9 \%$ & $5.3 \%$ \\
\hline Idaho & 108 & 61 & 42 & 82 & 63 & 120 & 51 & 57 & 59 & 75 & $-30.7 \%$ & $-4.0 \%$ \\
\hline Montana & 61 & 24 & 35 & 56 & 43 & 32 & 71 & 10 & 28 & 99 & $62.4 \%$ & $5.5 \%$ \\
\hline Nevada & 64 & 135 & 194 & 174 & 179 & 205 & 242 & 313 & 305 & 250 & $290.0 \%$ & $16.3 \%$ \\
\hline North Dakota & 93 & 37 & 73 & 72 & 72 & 194 & 35 & 67 & 113 & 31 & $-66.2 \%$ & $-11.3 \%$ \\
\hline Oregon & 27 & 29 & 28 & 46 & 65 & 42 & 71 & 50 & 50 & 65 & $142.8 \%$ & $10.4 \%$ \\
\hline South Dakota & 59 & 351 & 87 & 57 & 56 & 27 & 79 & 90 & 60 & 105 & $77.0 \%$ & $6.5 \%$ \\
\hline Utah & 124 & 7 & 150 & 94 & 152 & 203 & 129 & 266 & 220 & 290 & $133.5 \%$ & $9.9 \%$ \\
\hline Washington & 47 & - & 52 & 25 & 26 & 27 & 39 & 45 & 49 & 64 & $35.6 \%$ & $3.4 \%$ \\
\hline Wyoming & - & 125 & 98 & 71 & 69 & 67 & - & 147 & 78 & 75 & \#DIV/0! & \#\#\#\# \\
\hline Noridian Total & 53 & 52 & 69 & 69 & 79 & 77 & 84 & 91 & 100 & 113 & $113.2 \%$ & $8.8 \%$ \\
\hline PCPY & & $-2.2 \%$ & $33.6 \%$ & $0.2 \%$ & $13.7 \%$ & $-2.0 \%$ & $9.1 \%$ & $8.4 \%$ & $10.2 \%$ & $12.0 \%$ & & \\
\hline
\end{tabular}


Pain Physician: May/June 2020 23:S129-S147

Appendix Table 6 con't. Utilization of cervical/thoracic facet neurolysis rate per 100,000 Medicare beneficiaries (episodes) by 2016

Medicare carrier and state.

\begin{tabular}{|c|c|c|c|c|c|c|c|c|c|c|c|c|}
\hline State name & R2009 & R2010 & R2011 & R2012 & R2013 & R2014 & R2015 & R2016 & R2017 & R2018 & Change & GM \\
\hline \multicolumn{13}{|l|}{ Palmetto GBA } \\
\hline North Carolina & 48 & 71 & 51 & 60 & 50 & 33 & 78 & 59 & 97 & 113 & $133.4 \%$ & $9.9 \%$ \\
\hline South Carolina & 37 & 3 & 58 & 66 & 67 & 83 & 99 & 96 & 127 & 144 & $285.5 \%$ & $16.2 \%$ \\
\hline Virginia & 34 & 47 & 38 & 48 & 51 & 95 & 64 & 102 & 104 & 91 & $166.2 \%$ & $11.5 \%$ \\
\hline West Virginia & 48 & 63 & 83 & 82 & 110 & 118 & 121 & 115 & 140 & 134 & $180.1 \%$ & $12.1 \%$ \\
\hline Palmetto Total & 42 & 49 & 51 & 60 & 60 & 70 & 82 & 85 & 109 & 115 & $174.7 \%$ & $11.9 \%$ \\
\hline PCPY & & $18 \%$ & $5 \%$ & $16 \%$ & $0 \%$ & $17 \%$ & $18 \%$ & $3 \%$ & $29 \%$ & $5 \%$ & & \\
\hline \multicolumn{13}{|l|}{ Novitas } \\
\hline Arkansas & 246 & 199 & 159 & 145 & 216 & 312 & 348 & 377 & 334 & 338 & $37.3 \%$ & $3.6 \%$ \\
\hline Colorado & 80 & 51 & 89 & 57 & 108 & 142 & 142 & 173 & 184 & 229 & $187.5 \%$ & $12.4 \%$ \\
\hline Delaware & 14 & 13 & 52 & - & 12 & 95 & 80 & 133 & 41 & 109 & $693.4 \%$ & $25.9 \%$ \\
\hline $\begin{array}{l}\text { District } \\
\text { Columbia }\end{array}$ & - & - & 25 & 74 & - & - & 296 & 23 & 44 & 43 & \#DIV/0! & \#\#\#\# \\
\hline Louisiana & 74 & 84 & 85 & 97 & 123 & 128 & 166 & 134 & 171 & 174 & $134.0 \%$ & $9.9 \%$ \\
\hline Maryland & 50 & 69 & 69 & 73 & 72 & 98 & 138 & 129 & 114 & 125 & $150.5 \%$ & $10.7 \%$ \\
\hline Mississippi & 98 & 68 & 75 & 104 & 53 & 51 & 101 & 139 & 103 & 112 & $13.5 \%$ & $1.4 \%$ \\
\hline New Jersey & 32 & 42 & 36 & 44 & 54 & 79 & 102 & 75 & 88 & 109 & $237.0 \%$ & $14.5 \%$ \\
\hline New Mexico & 132 & 38 & 56 & 115 & 30 & 70 & 79 & 172 & 232 & 102 & $-22.2 \%$ & $-2.7 \%$ \\
\hline Oklahoma & 41 & 56 & 62 & 77 & 117 & 133 & 167 & 191 & 235 & 208 & $411.9 \%$ & $19.9 \%$ \\
\hline Pennsylvania & 27 & 28 & 22 & 43 & 39 & 41 & 47 & 63 & 48 & 64 & $140.6 \%$ & $10.2 \%$ \\
\hline Texas & 98 & 107 & 104 & 124 & 153 & 125 & 153 & 201 & 196 & 224 & $128.6 \%$ & $9.6 \%$ \\
\hline Novitas Total & 72 & 71 & 70 & 83 & 98 & 105 & 130 & 149 & 148 & 162 & $125.2 \%$ & $9.4 \%$ \\
\hline PCPY & & $-1.4 \%$ & $-0.9 \%$ & $18.6 \%$ & $17.2 \%$ & $7.5 \%$ & $23.8 \%$ & $14.8 \%$ & $-1.0 \%$ & $9.6 \%$ & & \\
\hline \multicolumn{13}{|l|}{ WPS } \\
\hline Indiana & 73 & 70 & 90 & 52 & 69 & 107 & 99 & 90 & 78 & 84 & $15.3 \%$ & $1.6 \%$ \\
\hline Iowa & 23 & 31 & 61 & 49 & 48 & 69 & 85 & 59 & 64 & 82 & $248.1 \%$ & $14.9 \%$ \\
\hline Kansas & 47 & 69 & 73 & 40 & 44 & 39 & 72 & 70 & 31 & 73 & $54.5 \%$ & $5.0 \%$ \\
\hline Michigan & 48 & 82 & 67 & 91 & 101 & 85 & 107 & 98 & 89 & 121 & $149.5 \%$ & $10.7 \%$ \\
\hline Missouri & 67 & 50 & 43 & 38 & 55 & 75 & 104 & 60 & 74 & 65 & $-3.4 \%$ & $-0.4 \%$ \\
\hline Nebraska & 36 & 29 & 64 & 63 & 35 & 34 & 26 & 57 & 79 & 124 & $242.5 \%$ & $14.7 \%$ \\
\hline WPS Total & 54 & 63 & 67 & 62 & 71 & 79 & 95 & 80 & 75 & 94 & $74.1 \%$ & $6.4 \%$ \\
\hline PCPY & & $17.9 \%$ & $5.1 \%$ & $-7.3 \%$ & $14.7 \%$ & $11.4 \%$ & $20.6 \%$ & $-16.0 \%$ & $-5.6 \%$ & $24.2 \%$ & & \\
\hline USA Total & 57 & 56 & 62 & 69 & 74 & 80 & 93 & 103 & 110 & 121 & $111.9 \%$ & $8.7 \%$ \\
\hline PCPY & & $-2.1 \%$ & $11.7 \%$ & $10.2 \%$ & $8.4 \%$ & $7.2 \%$ & $16.7 \%$ & $10.8 \%$ & $6.6 \%$ & $9.8 \%$ & & \\
\hline
\end{tabular}

